University of Louisville

ThinkIR: The University of Louisville's Institutional Repository

Electronic Theses and Dissertations

8-2018

\title{
A clinically relevant mouse model of cisplatin-induced kidney injury.
}

Cierra Nichole Sharp

University of Louisville

Follow this and additional works at: https://ir.library.louisville.edu/etd

Part of the Medical Pharmacology Commons, Medical Toxicology Commons, and the Pharmacy and Pharmaceutical Sciences Commons

\section{Recommended Citation}

Sharp, Cierra Nichole, "A clinically relevant mouse model of cisplatin-induced kidney injury." (2018). Electronic Theses and Dissertations. Paper 3063.

https://doi.org/10.18297/etd/3063

This Doctoral Dissertation is brought to you for free and open access by ThinkIR: The University of Louisville's Institutional Repository. It has been accepted for inclusion in Electronic Theses and Dissertations by an authorized administrator of ThinkIR: The University of Louisville's Institutional Repository. This title appears here courtesy of the author, who has retained all other copyrights. For more information, please contact thinkir@louisville.edu. 


\title{
A CLINICALLY RELEVANT MOUSE MODEL OF CISPLATIN-INDUCED KIDNEY
}

\author{
INJURY
}

\section{By}

\begin{abstract}
Cierra Nichole Sharp
B.A., Transylvania University, 2013

M.S., University of Louisville, 2016
\end{abstract}

\author{
A Dissertation \\ Submitted to the Faculty of the \\ School of Medicine of the University of Louisville \\ in Partial Fulfillment of the Requirements \\ for the Degree of \\ Doctor of Philosophy \\ in \\ Pharmacology and Toxicology \\ Department of Pharmacology and Toxicology \\ University of Louisville \\ Louisville, Kentucky
}

August 2018 



\title{
A CLINICALLY RELEVANT MOUSE MODEL OF CISPLATIN-INDUCED KIDNEY INJURY
}

\author{
By
}

\section{Cierra Nichole Sharp}

B.A., Transylvania University, 2013

M.S., University of Louisville, 2016

A Dissertation Approved on

May 17, 2018

By the following Dissertation Committee:

Gavin E. Arteel, Ph.D. Committee Member

Levi J. Beverly, Ph.D. Committee Member

Geoffrey J. Clark, Ph.D. Committee Member

Eleanor D. Lederer, M.D. Committee Member 


\section{DEDICATION}

I dedicate this dissertation to the educators who inspired me to pursue a Ph.D., and to the scientists before me whose work have made my project possible. To my cat, Chancellor Mangok of Eritrea- I'm getting my Ph.D. so that you can have all the catnip and toys you want. This is for you. 


\section{ACKNOWLEDGEMENTS}

I would like to thank my parents for letting me choose my own path in life, and for always being proud of me no matter what. To my friends and coworkers who have been by my side during this process- thank you for your emotional support and your scientific input. To Anna Lang, my best friend- your support in both my career and personal life are invaluable to me. I wish you nothing but happiness in life, love, and your career as Dr. Lang. 


\begin{abstract}
A CLINICALLY RELEVANT MOUSE MODEL OF CISPLATIN-INDUCED KIDNEY INJURY
\end{abstract}

Cierra Nichole Sharp

May 17, 2018

Cisplatin is a potent chemotherapeutic used for the treatment of many solid cancers, including testicular, ovarian, and lung cancer. Cisplatin causes many adverse side effects, of which nephrotoxicity leading to acute kidney injury is dose-limiting. Approximately $30 \%$ of patients will develop nephrotoxicity with cisplatin, and will either have their next dose of cisplatin lowered, skipped, or be switched to a less nephrotoxic chemotherapeutic altogether. These outcomes are not ideal when trying to treat cancer.

Previously, it was believed that patients could recover from cisplatin-induced acute kidney injury with little to no lasting effects, but recent longitudinal studies have shown this is not the case. Patients who develop acute kidney injury are ten times more likely to develop chronic kidney disease, which often requires dialysis and has an increased mortality rate. Unfortunately, there are no treatment options available for either acute kidney injury or chronic kidney disease. This may be due to the fact that the mouse model used to study cisplatin-induced kidney injury is not physiologically relevant to patients. The work featured in this dissertation aims to develop a clinically relevant 
mouse model of cisplatin-induced kidney injury by developing a cisplatin dosing regimen that recapitulates the type of dosing regimen patients receive. In order to further improve clinical relevancy, the effects of aging and cancer are also explored with this mouse model. The ultimate goal of this work is to use this model to identify a novel target and therapeutic strategy for the treatment of cisplatin-induced kidney injury. 
TABLE OF CONTENTS

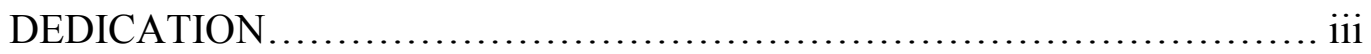

ACKNOWLEDGEMENTS ......................................... iv

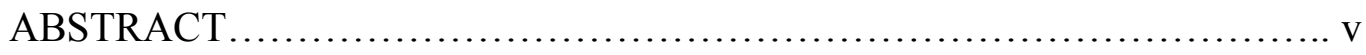

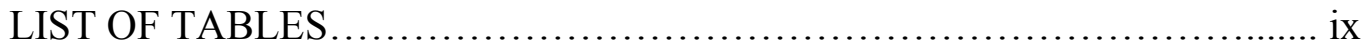

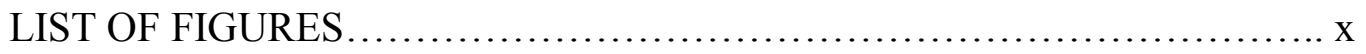

CHAPTER 1: BACKGROUND AND INTRODUCTION.............................. 1

CHAPTER 2: SUB-CLINICAL KIDNEY INJURY INDUCED BY REPEATED CISPLATIN ADMINISTRATION RESULTS IN PROGRESSIVE CHRONIC KIDNEY DISEASE .................................................. 21

CHAPTER 3: AGING DOES NOT LEAD TO INCREASED SUSCEPTIBILITY TO CISPLATIN-INDUCED KIDNEY INJURY OR EXACERBATED INJURY WITH REPEATED, LOW DOSE CISPLATIN ADMINISTRATION............... 57

CHAPTER 4: TREATMENT WITH REPEATED DOSING OF CISPLATIN CAUSES WORSENED FIBROSIS IN A MOUSE MODEL OF KRAS4BG12D LUNG ADENOCARCINOMA THAT CANNOT BE PREVENTED WITH

ERLOTINIB ..................................................... 86

SUMMARY AND DISCUSSION .................................. 117

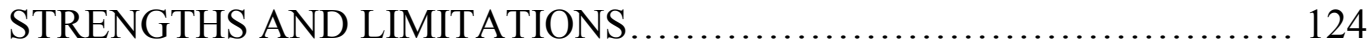

FUTURE DIRECTIONS ......................................... 127

REFERENCES .................................................... 133 


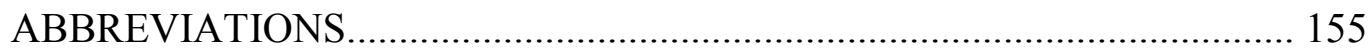

CURRICULUM VITAE............................................... 157 
LIST OF TABLES

TABLE 1.1: FAILED CLINICAL TRIALS FOR AKI RENOPROTECTIVE STRATEGIES..................................................................

TABLE 1.2: EXPERIMENTAL REPEATED DOSING REGIMENS OF

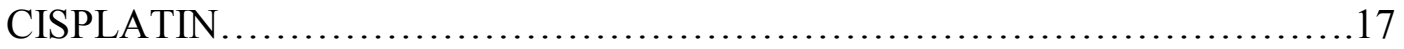

TABLE 2.1: PREPARATION OF TISSUE FOR PARAFFIN

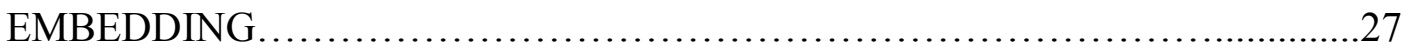

TABLE 2.2: HEMATOXYLIN AND EOSIN STAINING......................28

TABLE 2.3: PERIODIC ACID-SCHIFF STAINING .........................28

TABLE 5.1: OVERVIEW OF INJURY WITH THE REPEATED DOSING REGIMEN

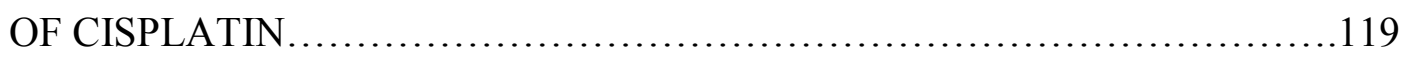

TABLE 5.2: DIFFERENCES BETWEEN 8 AND 40 WEEK OLD MICE TREATED WITH EITHER THE STANDARD OR REPEATED DOSING REGIMEN OF

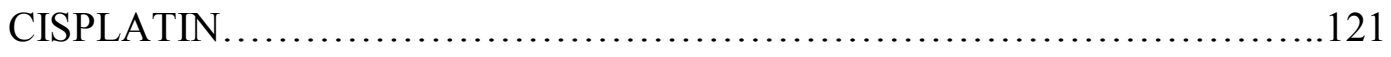

TABLE 5.3: DIFFERENCES IN INJURY OF NON CANCER AND CANCER MICE TREATED WITH THE REPEATED DOSING REGIMEN OF CISPLATIN...123 
LIST OF FIGURES

FIGURE 1.1: MECHANISMS OF MALADAPTIVE REPAIR ......................9

FIGURE 2.1: REPEATED DOSING REGIMEN OF CISPLATIN...................24

FIGURE 2.2: ALLOCATION OF KIDNEY TISSUE............................25

FIGURE 2.3: MARKERS OF KIDNEY FUNCTION AND INJURY DURING AND AFTER REPEATED CISPLATIN TREATMENT ..............................33

FIGURE 2.4: TUBULAR INJURY DURING AND AFTER REPEATED CISPLATIN

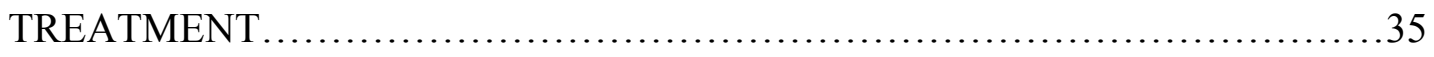

FIGURE 2.5: INFLAMMATORY CYTOKINE AND CHEMOKINE LEVELS DURING AND AFTER REPEATED CISPLATIN TREAMENT ............................37

FIGURE 2.6: TOTAL MACROPHAGES AND MACROPHAGE SUBTYPES DURING AND AFTER REPEATED CISPLATIN TREATMENT. 39

FIGURE 2.7: PRO-FIBROTIC GROWTH FACTOR PRODUCTION DURING AND AFTER REPEATED CISPLATIN TREATMENT.

FIGURE 2.8: DEVELOPMENT OF INTERSTITIAL FIBROSIS DURING AND AFTER REPEATED CISPLATIN TREATMENT

FIGURE 2.9: INDICES OF GLOMERULAR DAMAGE DURING AND AFTER REPEATED CISPLATIN TREATMENT ...................................46 
FIGURE 2.10: MARKERS OF ENDOTHELIAL DYSFUNCTION AND DAMAGE DURING AND AFTER REPEATED CISPLATIN TREATMENT 48

FIGURE 3.1: STANDARD DOSING REGIMEN OF CISPLATIN 60

FIGURE 3.2: REPEATED DOSING REGIMEN OF CISPLATIN 60

FIGURE 3.3: SURVIVAL AND WEIGHT LOSS WITH THE STANDARD DOSING REGIMEN OF CISPLATIN.

FIGURE 3.4: CHANGES IN KIDNEY FUNCTION AND INJURY MARKERS WITH THE STANDARD DOSING REGIMEN OF CISPLATIN .65

FIGURE 3.5: CHANGES IN INFLAMMATORY CYTOKINE AND CHEMOKINE LEVELS WITH THE STANDARD DOSING REGIMEN OF CISPLATIN ...67

FIGURE 3.6: ENDOPLASMIC RETICULUM STRESS AND APOPTOSIS WITH THE STANDARD DOSING REGIMEN OF CISPLATIN .69

FIGURE 3.7: SURVIVAL AND WEIGHT LOSS WITH THE REPEATED DOSING REGIMEN OF CISPLATIN. 71

FIGURE 3.8: CHANGES IN KIDNEY FUNCTION AND INJURY MARKERS WITH THE REPEATED DOSING REGIMEN OF CISPLATIN. .73

FIGURE 3.9: CHANGES IN INFLAMMATORY CYTOKINE AND CHEMOKINE LEVELS WITH THE REPEATED DOSING REGIMEN OF CISPLATIN...75

FIGURE 3.10: FIBROSIS DEVELOPS WITH THE REPEATED DOSING REGIMEN OF CISPLATIN .77 
FIGURE 3.11: IMMUNE CELLS IN THE KIDNEY WITH THE REPEATED DOSING REGIMEN OF CISPLATIN.......................................80

FIGURE 4.1: INDUCIBLE TRANSGENIC MODEL OF MUTANT KRAS LUNG ADENOCARCINOMA.............................................91

FIGURE 4.2: CISPLATIN AND ERLOTINIB DOSING REGIMEN.....92

FIGURE 4.3: TREATMENT WITH REPEATED ADMINISTRATION OF CISPLATIN DECREASES OVERALL SURVIVAL IN CANCER MICE. .95

FIGURE 4.4: KIDNEY FUNCTION AND INJURY IN NON CANCER AND CANCER MICE TREATED WITH THE REPEATED DOSING REGIMEN OF CISPLATIN

FIGURE 4.5: CHANGES IN INFLAMMATORY CYTOKINE AND CHEMOKINE LEVELS IN NON CANCER AND CANCER MICE TREATED WITH THE REPEATED DOSING REGIMEN OF CISPLATIN. 99

FIGURE 4.6: DEVELOPMENT OF FIBROSIS IN NON CANCER AND CANCER MICE TREATED WITH THE REPEATED DOSING REGIMEN OF CISPLATIN 101

FIGURE 4.7: EPIDERMAL GROWTH FACTOR RECEPTOR ACTIVATION AND DOWSTREAM SIGNALING PATHWAY ACTIVATION IN NON CANCER AND CANCER MICE TREATED WITH THE REPEATED DOSING REGIMEN OF CISPLATIN 103 
FIGURE 4.8: OVERALL SURVIVAL AND WEIGHT LOSS WITH CISPLATIN+ERLOTINIB ..................................105

FIGURE 4.9: KIDNEY INJURY AND LOSS OF FUNCTION WITH CISPLATIN+ERLOTINIB ......................................107

FIGURE 4.10: CHANGES IN INFLAMMATORY CYTOKINE AND CHEMOKINE LEVELS WITH CISPLATIN+ERLOTINIB ......................109

FIGURE 5.1: DEVELOPMENT OF RENAL FIBROSIS IN FVB/N AND C57BL/6J MICE

FIGURE 5.2: ERLOTINIB AFTER REPEATED DOSING OF CISPLATIN

FIGURE 5.3: ALTERATIONS IN BMAL1, AN ESSENTIAL REGULATOR OF CIRCADIAN RHYTHMS 


\section{CHAPTER 1}

\section{BACKGROUND AND INTRODUCTION}

\subsection{Cisplatin}

Cisplatin (cis-diamminedichloridoplatinum [II]) is one of the few platinum-based chemotherapeutics used clinically, and arguably one of the most successful chemotherapeutics to be used $(2,3)$. Its potential use in cancer treatment began in the 1960s when Barret Rosenberg was studying E.Coli cellular division and the role electrical currents played in proper cell division $(2,4)$. In his studies, inert platinum electrodes immersed in ammonium chloride buffer inhibited cell division, and this inhibition led to the hypothesis that the platinum salt produced by hydrolysis of the platinum electrode may have potential as a cancer therapeutic (5). Initial studies in Swiss white mice with Sarcoma confirmed that cisplatin could cure cancer (2).

Since its FDA approval in the early 1970s, cisplatin has been used in the treatment of many solid tumor cancers $(2,6)$. Cisplatin is efficacious for the treatment of ovarian cancer, head and neck cancer, bladder cancer, cervical cancer, melanoma, certain lymphomas, and non-small cell lung cancer (NSCLC) $(2,6)$. The therapeutic dosing regimen for treatment of these cancers with cisplatin varies greatly. For example, patients with ovarian cancer receive one, high dose $\left(90-270 \mathrm{mg} / \mathrm{m}^{2}\right)$ of cisplatin intravenously $(2,7)$. However, for head and neck cancers, cisplatin is often used as a 
chemosensitization step prior to treatment with radiation. Furthermore, patients with NSCLC often receive $60-100 \mathrm{mg} / \mathrm{m}^{2}$ cisplatin in combination with other antineoplastic agents once every 21 days for multiple cycles (2)

Cisplatin's antitumor efficacy is based on its ability to induce apoptosis in cancer cells $(2,6,8)$. Once in the bloodstream, cisplatin can enter into cells by simple diffusion, or by uptake via copper transport proteins. Inside the cell, cisplatin encounters a low chloride concentration, and therefore one of its chloro-ligands is rapidly replaced with water. This replacement gives cisplatin a positive charge, and this intermediate species can readily bind to guanine bases of DNA to form adducts (8). The formation of these DNA adducts initiates apoptotic signaling pathways, resulting in cell death.

A general downside to use of chemotherapeutics (including cisplatin) is that the mechanism of action for killing rapidly dividing cancer cells also kills normal, rapidly

dividing cells in the body, including cells lining the gastrointestinal tract (2). Due to this chemotherapeutics, like cisplatin, cause nausea and hair loss. However, cisplatin has several other severe toxicities. Of note, cisplatin has a high rate of ototoxicity, and older patients run a risk of hematological toxicity with cisplatin (9). However, the doselimiting side effect of cisplatin is nephrotoxicity.

\subsection{Cisplatin-Induced Nephrotoxicity}

The mechanisms involved in cisplatin nephrotoxicity are complex. A large portion of cisplatin binds to albumin in the blood, and bound cisplatin is freely filtered through glomeruli in the kidney $(2,9,10)$. Filtered cisplatin is taken up by organic cation transporter $2(\mathrm{OCT} 2)$ and copper transporter $1(\mathrm{Ctr} 1)$ in proximal tubule epithelial cells, 
leading to a five time higher concentration of cisplatin in these cells compared to the serum (11-13). Once inside tubular cells, cisplatin has a direct toxic effect via DNA alkylation and subsequent activation of apoptotic signaling pathways $(9,10,14,15)$. In addition, cisplatin can be converted to highly reactive, thiol conjugate intermediates. These intermediates readily bind to DNA, RNA, and proteins leading to enhanced renal tubular cell death $(9,10,14,15)$. Whereas cell death is therapeutic in the context of cancer, the high level of tubular cell death causes severe nephrotoxicity.

\subsection{Pathophysiology of Cisplatin-Induced Nephrotoxicity}

The two main forms of cell death involved in cisplatin nephrotoxicity are apoptosis and necrosis $(9,10,14,15)$. Specifically, cisplatin activates the intrinsic apoptotic signaling pathway (9). Injury to tubular cells from cisplatin leads to an accumulation of Bax, a BCL2 pro-apoptotic protein, in the mitochondria (16). This accumulation of Bax causes the release of cytochrome $\mathrm{c}$, and downstream activation of initiator and effector caspases, particularly the cleavage and activation of Caspase-3 (9).

Necrosis caused by cisplatin is largely mediated by inflammation, a major contributor to the pathophysiology of cisplatin-induced nephrotoxicity $(9,10,14,15)$. TNF $\alpha$ is produced by resident kidney cells, and levels increase in the kidney, blood, and urine after kidney injury $(17,18)$. The release of TNF $\alpha$ leads to the recruitment of neutrophils and other immune cell types to the site of injury $(9,10)$.

While most of the mechanisms of injury associated with cisplatin-induced nephrotoxicity are tubule and immune-cell focused, subsequent damage to renal vasculature via endothelial dysfunction leads to irregular vasoconstriction and further 
damage to the kidney (1). In addition, cisplatin is directly toxic to endothelial cells which comprise blood vessels in the kidney (1). The culmination of this multi-faceted injury causes loss of blood flow to the kidney, and creates a hypoxic environment which can exacerbate stress and cell death within tubular cells (1). Damage to both the vasculature and loss of tubular cells leads to an overall loss of renal tissue, and subsequent loss of kidney function. With cisplatin, this loss of function is severe and occurs over a period of only a few days, and in doing so, is classified as acute kidney injury (AKI) (19).

\subsection{Acute Kidney Injury}

Clinically, AKI is diagnosed by measures of serum creatinine ( $\mathrm{SCr}$ ), glomerular filtration rate (GFR), and urine output $(19,20)$. For diagnosis of cisplatin-induced AKI, SCr is commonly used. According to RIFLE criteria, a patient diagnosed with AKI will have a doubling in their baseline SCr levels, or a $>50 \%$ decrease in $\operatorname{GFR}(19,20)$. Ten to $15 \%$ of patients with AKI may have severe loss of function requiring dialysis- a costly medical treatment that does not repair kidney injury sustained. Furthermore, AKI has a $50 \%$ mortality rate, and this mortality rate varies based on the cohort of patients being studied (21). For example, the mortality rate from AKI in patients in the ICU is closer to $80 \%(22,23)$.

There are many different forms of AKI, and these can be divided into three categories: (1) prerenal, (2) intrinsic, and (3) post renal (9). Cisplatin causes intrinsic injury, and cisplatin nephrotoxicity accounts for $19 \%$ of the total cases of AKI, making it a major health concern (21) Approximately one third of patients will develop AKI after a single dose of cisplatin, and the amount of cisplatin administered, as well as the frequency that the dose is given are the two greatest risk factors for developing cisplatin- 
induced AKI (24-26) Gender (being female), advanced age, smoking, and pre-existing renal dysfunction are also contributing factors to increased risk of developing cisplatininduced AKI. These risk factors also contribute to poor long-term outcomes associated with cisplatin treatment-namely the development of chronic kidney disease (CKD).

\subsection{Development of CKD after AKI}

Until recently, AKI was thought to be a reversible syndrome, and patients that did not require dialysis or die from AKI would have a return to normal kidney function once the initial injury was resolved (1). However, large-scale longitudinal studies from the past decade suggest that AKI can result in permanent kidney damage associated with CKD (24-26). Patients with AKI have a ten time greater risk of developing CKD, and this risk is further increased with severe AKI, as well as multiple instances of AKI. Not only have studies shown an increased risk for developing $\mathrm{CKD}$ after $\mathrm{AKI}$, but experimental data have highlighted that AKI can progress to CKD. In fact, it is estimated that $\sim 20 \%$ of all patients with AKI will progress to CKD in as little as 18-24 months (27).

\subsection{The Progression of AKI to CKD}

While AKI has been indicated as an independent risk factor for the development of CKD, the idea that AKI can cause CKD in humans has been debated due to several confounding factors, such as lack of temporal cause/outcome and pre-existing comorbidities that may pre-dispose patients to the development of CKD (28). However, both experimental data and human studies indicate that AKI can lead to CKD. Healthy patients who develop AKI have abnormal renal function long after the initial AKI insult is treated or resolved. This finding has been recapitulated in two separate studies in 
young patients (average age 31-32 years old) who were healthy before their AKI (29).

Furthermore, patients who had severe AKI showed long-term abnormalities in their ability to concentrate urine (29). A case study from Sasaki et al reported on two patients who developed CKD after cisplatin treatment (30). Both patients were treated with 80 $\mathrm{mg} / \mathrm{m}^{2}$ cisplatin for esophageal cancer. These patients had a spike in their $\mathrm{SCr}$ levels that required immediate hemodialysis. Several months later, a kidney biopsy was taken and revealed the development of glomerulosclerosis, lymphocyte infiltration into the kidney, and interstitial fibrosis (30).

The interference of other comorbidities contributing to the development of CKD can be accounted for by studying pediatric cancer patients (31). The pediatric cancer population is the ideal demographic for studying the development of CKD after cisplatininduced AKI. This population is relatively healthy, and has fewer risk factors than the adult population. Therefore, better conclusions can be made about the true risk of developing CKD after cisplatin-induced AKI. In one study of children that received cisplatin for a variety of cancers, approximately $50 \%$ showed signs of nephrotoxicity four years post-treatment, and $36.4 \%$ of these children maintained a decreased GFR indicative of CKD (24). Skinner et al also reported that $11 \%$ of children who had received cisplatin or carboplatin had decreased GFR ten years post-treatment (32). Taken together, these studies highlight the long-lasting effects on the kidney after cisplatin treatment. However, the mechanisms that lead to the development of CKD after are not fully understood in humans, and thus animal models have become increasingly important to understand this disease progression. 
Animal studies using ischemia-reperfusion (I/R) injury have been crucial for understanding the underlying processes involved in the progression from AKI to CKD ( 1 , 9). Ischemia-reperfusion results in an acute but temporary loss of blood flow to the kidney, causing high levels of damage. Studies with this experimental model of injury have indicated that there is residual renal structural damage even after the initial AKI is no longer detectable by changes in SCr. Furthermore, these animals often develop interstitial fibrosis, with a persistent reduction in capillary density within the kidney (1). These processes which remain altered after AKI are known contributors to CKD development.

\subsection{Mechanisms of Maladaptive Repair}

After injury occurs to the kidney, the kidney may undergo adaptive repair processes that restore kidney function. This involves the ability of injured tubular cells to regenerate, which is mediated by an immune cell response and resolution of inflammation that occurs with AKI (1). While neutrophils are the first immune cell type recruited to the site of injury, their role in repair and injury remain unclear. Shortly after recruitment of neutrophils, however, M1 subtype macrophages are recruited to the site of injury (9). M1 macrophages are pro-inflammatory in nature, and amplify initial injury (33-35). Over time, these M1 macrophages adopt an M2 phenotype. M2 macrophages are vital for the removal of both dead neutrophils and other cellular debris after initial injury, contributing to renal repair.

Adaptive repair relies on increases in circulating and endogenous compounds that promote cell proliferation and resolution of inflammation (1). M2 macrophages release growth factors, fibronectin, and Wnt-7b that aid in tubular repair and subsequent 
proliferation of tubule cells (35). Furthermore, damage to vasculature leads to the release of heme oxygenase 1 (HO-1), which initiates an anti-inflammatory response (1). In addition to these processes, endothelial repair and regeneration is also important for adaptive repair. However, with $\mathrm{I} / \mathrm{R}$ injury in rats, there is long-term reduction in peritubular capillary density with persistent renal hypoxia, leading to injury progression (1). These data highlight that perhaps there is never full recovery from injury, and alludes to the worsening of renal injury leading to fibrosis and CKD even after kidney function levels return to baseline. The mechanisms contributing to the development of CKD after AKI are termed "maladaptive repair" (Figure 1.1) 


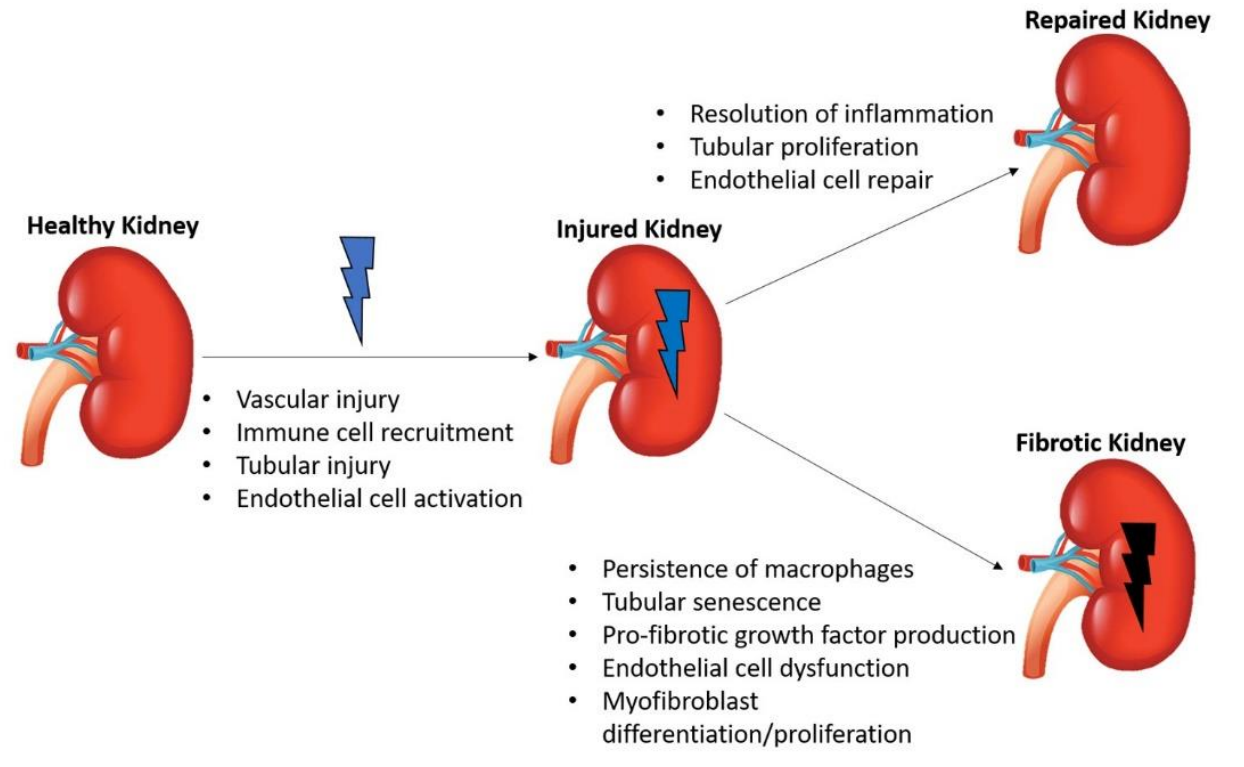

Adapted from Ferenbach et al (1)

Figure 1.1. Mechanisms of maladaptive repair. After the kidney is injured, the kidney is able to resolve inflammatory processes, and promote tubular proliferation/ endothelial cell repair. However, if injury persists or there are multiple instances of injury, tubular epithelial cells are prone to senescence. In this senescent state, cell produce pro-fibrotic growth factors which can stimulate myofibroblast differentiation and proliferation, ultimately resulting in fibrosis. 
As previously discussed, the risk of developing CKD after AKI in patients increases with recurrent episodes of AKI. Recurrent injury to tubular cells can promote the development of fibrosis, indicative of CKD. Repeated dosing studies with diphtheria toxin in mice with tubular epithelial cells expressing the diphtheria toxin receptor indicate that injury specific to tubular epithelia results in persistent inflammation, microvascular rarefaction, fibrogenesis with subsequent pro-fibrotic growth factor production, and the development of glomerulosclerosis $(36,37)$. Furthermore, sub-lethal injury to tubular cells induces premature cellular senescence and the inability of these cells to proliferate and regenerate after injury $(36,37)$. Tubular cells in their dedifferentiated, senescent state continue to produce pro-fibrotic growth factors, exacerbating damage to the kidney vasculature (1). Production of pro-fibrotic growth factors also stimulates myofibroblast differentiation and proliferation, resulting in high levels of collagen production associated with fibrosis (1).

Other perturbations in adaptive repair responses can cause progressive injury. Of note, M1 macrophages may not polarize to M2 with chronic injury, thus prolonging the pro-inflammatory response. Ultimately, maladaptive repair processes lead to the development of renal fibrosis and further damage to renal vasculature, causing progressive loss of renal function and loss of blood flow- both of which are heavily involved in CKD.

\subsection{Chronic Kidney Disease}

Chronic kidney disease is clinically characterized by decreased kidney function as measured by SCr or GFR, presence of proteinuria, and presence of albuminuria for more than three months (38). CKD is a slow, progressive disease that is often asymptomatic in 
its early stages. However, as the disease progresses, interstitial fibrosis and glomerulosclerosis begin to develop leading to a decline in kidney function (38). As interstitial fibrosis worsens, tubule cells in the kidney are separated from peritubular capillaries. This results in a lack of blood flow to the tubule cells, depriving them of oxygen and nutrients, while at the same hindering them from excreting toxins and waste products out of the cell (38). Due to the progressive nature of the disease, many patients will require dialysis on a regular basis, and these patients are more likely to progress to end stage renal disease $(\mathrm{ESRD})(27,38)$. Controlling for comorbidities, there are about 400 deaths per million people from CKD alone (27).

\subsection{Clinical Strategies for Treating Cisplatin-Induced Kidney Injury}

Patients with either AKI or CKD often have increased mortality rates and may require dialysis and, unfortunately, there are no options available for the treatment of either disease. The goal for treatment of cisplatin-induced kidney injury would be to protect the kidney from injury altogether- highlighting the need for renoprotective therapy options. However, only one drug is available for cisplatin-induced nephrotoxicity. Amifostine was first FDA approved as a renoprotective agent in the early 2000s because of its ability to inactivate the nephrotoxic metabolites of cisplatin within the cell (39). In Phase III clinical trials in patients with ovarian cancer, amifostine lowered the rate of cisplatin nephrotoxicity from $33 \%$ to $10 \%(7,40)$. However, the cisplatin dosing regimen for treating ovarian cancers greatly differs from the dosing regimen used to treat other forms of cancer. Patients with ovarian cancer are administered $90-270 \mathrm{mg} / \mathrm{m}^{2}$ of cisplatin once. Most solid tumor cancers treated with cisplatin use lower doses of cisplatin given 
repeatedly. The use of amifostine with this type of dosing regimen exacerbates nephrotoxicity (41). Due to this, amifostine is rarely used in combination with cisplatin.

Few interventions are available clinically to protect against the development of AKI. As a precaution, patients receive saline infusions prior to cisplatin treatment to protect against the development of AKI. However, even with this precaution in place, approximately $30 \%$ of patients still develop AKI. For patients that have increased $\mathrm{SCr}$ levels during cisplatin treatment, the oncologist has limited options for protecting from further injury. Oncologists may choose to either switch the patient to a less nephrotoxic albeit less potent platinum-based therapy (i.e. carboplatin or oxaliplatin), or skip/lower the next dose of cisplatin. Neither of these options are ideal for the treatment of cancer, which requires an aggressive therapy for a curative effect.

\subsection{Lack of Therapies for Cisplatin-Induced Kidney Injury}

Although many pharmacological strategies have been indicated as having renoprotective potential, few have succeeded in clinical trials (Table 1.1) $(42,43)$. This may be due to the fact that clinical trials for new AKI therapies usually have low enrollment and poor endpoint measures for drug efficacy $(44,45)$. Clinical trials for experimental AKI therapies have a low enrollment number because many patients with AKI have comorbidities (such as pre-existing CKD or diabetes) that can alter their outcome to the drug being tested $(28,44)$. Without a proper sampling group, the statistical power of clinical trials is poor. In the PICARD study, $30 \%$ of patients screened for AKI clinical trials had CKD, 29\% had diabetes, and many had extrarenal organ failure (46). These comorbidities contribute to failed treatment regimens, especially when the endpoint of the clinical trial is mortality. 


\begin{tabular}{|c|c|c|c|}
\hline Compound/Therapy & Mechanism of Action & Outcome Measure & Results \\
\hline Selenium & $\begin{array}{l}\text { Antioxidant to decrease oxidative } \\
\text { damage }\end{array}$ & Restoration of antioxidant levels & Inconclusive \\
\hline $\begin{array}{l}\text { Selenium + Vitamin C + Vitamin } \\
\text { E }\end{array}$ & $\begin{array}{l}\text { Antioxidant to decrease oxidative } \\
\text { damage }\end{array}$ & Decrease in malondialdehyde & Inconclusive \\
\hline ABT-719 & $\begin{array}{l}\text { Alpha melanocortin } 1 \text { receptor } \\
\text { agonist to protect from AKI } \\
\text { resulting from cardiac surgery }\end{array}$ & $\begin{array}{l}\text { Number of patients that develop } \\
\text { AKI; NGAL and KIM-1; Need for } \\
\text { renal replacement therapy }\end{array}$ & $\begin{array}{l}\text { No difference } \\
\text { in rates of AKI; } \\
\text { no difference } \\
\text { in NGAL or } \\
\text { KIM-1 }\end{array}$ \\
\hline Heme-arginate & $\begin{array}{l}\text { Induction of HO-1 to decrease } \\
\text { oxidative damage }\end{array}$ & $\begin{array}{l}\text { HO-1 upregulation; urine } \\
\text { production }\end{array}$ & $\begin{array}{l}\text { No difference } \\
\text { in renal } \\
\text { function due } \\
\text { to low } \\
\text { statistical } \\
\text { power }\end{array}$ \\
\hline AC607 & $\begin{array}{l}\text { Bone marrow-derived } \\
\text { mesenchymal stem cells for } \\
\text { prevention of cardiac surgery- } \\
\text { induced AKI }\end{array}$ & $\begin{array}{l}\text { Time to return to baseline } \\
\text { creatinine; length of hospital } \\
\text { stay; need for renal replacement } \\
\text { therapy }\end{array}$ & $\begin{array}{l}\text { Study } \\
\text { terminated } \\
\text { due to no } \\
\text { improvement } \\
\text { in any } \\
\text { outcome } \\
\text { measures } \\
\text { examined }\end{array}$ \\
\hline
\end{tabular}

Table 1.1. Failed clinical trials for AKI renoprotective strategies. Although some renoprotective therapies have made it to early stage clinical trials, few have advanced beyond this point.

In addition, the endpoint measure for many AKI studies is $\mathrm{SCr}$. However, $\mathrm{SCr}$ is a poor biomarker and does not truly reflect kidney function. Serum creatinine levels can be affected by age, gender, and muscle mass; therefore even baseline levels are not reflective of kidney function $(44,47,48)$. Finally, changes in SCr usually do not occur until there is $50 \%$ nephron loss- a relatively high level of damage to the kidney.

Aside from poor clinical trial design, there is a lack of a physiologically relevant animal models to study cisplatin-induced AKI. The commonly used mouse model of 
cisplatin-induced kidney injury consists of administering one, very high dose of cisplatin $(10-30 \mathrm{mg} / \mathrm{kg})$ that causes mice to die 3-4 days after injection (49-51). This dosing regimen does not take into consideration that patients often receive repeated, lower doses of cisplatin to maintain therapeutic efficacy while curtailing nephrotoxic side effects. Furthermore, this model does not allow long-term studies of renal outcomes with cisplatin pertaining to the development of CKD. Combined, poor clinical trial design and lack of relevant pre-clinical models provide insight into why there are no therapeutic options for treatment of cisplatin-induced kidney injury.

\subsection{Current Limitations in the Experimental Model of Cisplatin-Induced AKI}

Addressing the limitations of the established mouse model of cisplatin-induced AKI is essential for identifying new therapeutic targets. In particular, developing a dosing regimen that better recapitulates the therapeutic regimen patients receive can provide better mechanistic insight into injury associated with repeated dosing of cisplatin. Concurrently, including comorbidities such as age and cancer in the mouse model would improve the relevancy of the model, as only patients with cancer receive cisplatin, and the median age of all cancer diagnoses is 65 years old (52).

During normal aging, studies in mice and humans have indicated that physiological changes in the kidney occur over time, leading to a progressive decline in kidney function. In humans, this process begins around age 30 (53). In addition, inflammatory and immune function within the kidney is altered with aging. Combined, these changes could increase susceptibility to cisplatin-induced AKI. Furthermore, treatment of cancer has improved greatly over the last decade, meaning that there is now a rising population of patients who will have to deal with long-term outcomes associated with cisplatin 
treatment. Thus, developing a mouse model to combine both a clinically relevant dosing regimen of cisplatin and to study the effects cancer and aging may have on altering injury outcomes would provide comprehensive insight into cisplatin-induced AKI and its progression to CKD.

\subsection{Developing a Clinically Relevant Mouse Model of Cisplatin-Induced Kidney \\ Injury}

Several groups have developed more relevant cisplatin dosing regimens in which mice are given the drug repeatedly and at lower doses to recapitulate how patients receive cisplatin (Table 1.2). Torres et al demonstrated that administering $15 \mathrm{mg} / \mathrm{kg}$ cisplatin, followed by a second dose two weeks later, led to changes in kidney function indicative of AKI (54). This loss of kidney function was accompanied by high levels of apoptotic cell death, and slight changes to glomerular capsules which correlated to a decrease in GFR. However, there was little collagen accumulation, no increase in macrophage activity, and no increase in myofibroblast proliferation suggesting no fibrosis was occurring (54).

Ravichandran et al injected 8-10 week old C57BL6 mice with $10 \mathrm{mg} / \mathrm{kg}$ cisplatin once a week for four weeks. At the end of this dosing regimen, there was a significant increase in both blood urea nitrogen (BUN) and SCr levels in addition to moderate levels of apoptosis and necrosis (55). Collagen accumulation was present at the endpoint of the four week study, but there was no overt fibrosis. Similarly, Katagiri et al injected 7-8 week old C57BL6 mice once a week for three weeks with $10 \mathrm{mg} / \mathrm{kg}$ cisplatin. A week after the final dose, there was a significant increase in BUN and $\mathrm{SCr}$ (56). Further analysis showed loss of brush border and acute tubular necrosis, which are indicative of tubule damage. Furthermore, Katagiri et al. confirmed the presence of fibrosis via Masson's Trichrome 
staining and an increase in both $T g f \beta$ and $\alpha S m a$ mRNA levels (56). However, most mice were unable to survive the entirety of the dosing, suggesting that the accumulation of cisplatin caused severe nephrotoxicity.

The studies described here highlight that AKI can develop with repeated administration of cisplatin, but the ability to use these models to study long-term effects associated with the development of CKD after cisplatin-induced AKI are limited by poor survival or lack of fibrosis. To address these limitations, our lab has developed a repeated dosing regimen that causes fibrosis with a $100 \%$ survival rate (Table 1.2) $(50,51)$. This dissertation focuses on using this repeated dosing regimen as a basis for building a clinically relevant mouse model of cisplatin-induced kidney injury in order to identify novel therapeutic targets. 


\begin{tabular}{|c|c|c|c|c|}
\hline Reference & $\begin{array}{l}\text { Dose of } \\
\text { Cisplatin Used } \\
\text { (mg/kg) }\end{array}$ & $\begin{array}{l}\text { Frequency of } \\
\text { Dose }\end{array}$ & Mice Used & Results \\
\hline Torres et al. & 15 & $\begin{array}{l}\text { Two doses with } \\
\text { a } 2 \text { week } \\
\text { period } \\
\text { between each } \\
\text { dose }\end{array}$ & $\begin{array}{l}10 \text { week old } \\
\text { C57BL/6J }\end{array}$ & $\begin{array}{l}\text { Mild fibrosis, } \\
\text { long-term } \\
\text { survival, } \\
\text { decreased GFR }\end{array}$ \\
\hline $\begin{array}{l}\text { Ravichandran } \\
\text { et al. }\end{array}$ & 10 & $\begin{array}{l}\text { One dose a } \\
\text { week for } 4 \\
\text { weeks }\end{array}$ & $\begin{array}{l}\text { 8-10 week old } \\
\text { C57BL/6J }\end{array}$ & $\begin{array}{l}\text { Mild fibrosis } \\
\text { with high levels } \\
\text { of apoptotic } \\
\text { cell death }\end{array}$ \\
\hline Katagiri et al. & 10 & $\begin{array}{l}\text { One dose a } \\
\text { week for } 3 \\
\text { weeks }\end{array}$ & $\begin{array}{l}\text { 7-8 week old } \\
\text { C57BL/6J }\end{array}$ & $\begin{array}{l}\text { Overt fibrosis; } \\
\text { mice died } \\
\text { within } 4 \text { weeks }\end{array}$ \\
\hline Sharp et al. & 7 & $\begin{array}{l}\text { One dose a } \\
\text { week for } 4 \\
\text { weeks }\end{array}$ & 8 week old FVB & $\begin{array}{l}\text { Overt fibrosis; } \\
\text { long-term } \\
\text { survival }\end{array}$ \\
\hline
\end{tabular}

Table 1.2. Experimental, repeated dosing regimens of cisplatin. Although the amount of cisplatin and how often cisplatin is given varies between these models, repeated administration of cisplatin results in varying degrees of fibrosis, indicative of CKD. 


\section{OVERALL GOALS AND SPECIFIC AIMS}

While the development of therapies for cisplatin-induced kidney injury has been a field of study for several decades, there are still no clinically approved agents. The lack of therapies may be due to poor clinical trial design and outcome predictors, as well as a need for a more clinically relevant pre-clinical mouse model for testing potential renoprotective agents. The current, experiment mouse model of cisplatin-induced AKI consists of injecting mice with one, high dose of cisplatin $(10-30 \mathrm{mg} / \mathrm{kg})$ which causes mice to become moribund 3-4 days after the initial injection. Patients receive cisplatin in low doses over an extended period of time to avoid nephrotoxicity, and the increased risk of mortality associated with it. Thus, it is crucial to develop a dosing regimen that better mimics the dosing regimen patients receive. In addition, an ideal model of cisplatininduced kidney injury would better mimic patient characteristics via examining the effects of cancer and aging in the model.

The overall goal of this dissertation is to establish a clinically relevant mouse model of cisplatin-induced kidney injury that can be used to examine long-term outcomes of cisplatin nephrotoxicity. In addition, this model would be essential for monitoring disease progression from AKI to CKD. The outcomes of this study would aid in identifying novel therapeutic targets for the treatment of cisplatin-induced kidney injury. The specific aims of this project are as follows:

\section{Aim 1: Develop a clinically relevant dosing regimen of cisplatin in mice that models the progression of cisplatin-induced AKI to CKD.}

Repeated, low dose cisplatin administration in mice causes interstitial fibrosis, which is indicative of CKD. CKD is a progressive disease with many other pathologies 
in addition to interstitial fibrosis, including endothelial dysfunction, chronic inflammation, and glomerular pathologies. Mice treated with our repeated cisplatin dosing regimen develop the aforementioned pathologies six months post-treatment. Thus, we have developed a bona fide model of cisplatin-induced CKD.

\section{Aim 2: Determine if aging increases susceptibility to AKI/ causes worsened kidney injury with the clinically-relevant dosing regimen of cisplatin.}

It is hypothesized that aging is a risk factor for developing more severe nephrotoxicity and exacerbated kidney injury due to physiological changes that occur during normal aging. However, the clinical data to support this hypothesis is limited by the fact that older patients that receive cisplatin have comorbidities that may predispose them to AKI or CKD. Animal studies to explore the relationship between aging and worsened renal injury from cisplatin are often hindered by the fact that mice used are usually extremely old (24 months), while most cancer diagnoses are in middle-aged patients (55-65 years old). To improve on this, we utilized 8 and 40 week old mice, and treated them with either the standard or repeated dosing regimen of cisplatin $(25 \mathrm{mg} / \mathrm{kg}$ once or $7 \mathrm{mg} / \mathrm{kg}$ once a week for 4 weeks, respectively) to determine the severity of cisplatin nephrotoxicity. Our data indicate that aging does not have a major effect on renal injury outcomes, and suggests that the risk of cisplatin-induced kidney injury may not be as greatly affected by advanced age as previously believed.

\section{Aim 3: Increase clinical relevancy of the newly established model of cisplatin- induced kidney injury by incorporating cancer.}


Only patients who have cancer will receive cisplatin, thus incorporating cancer into our repeated dosing model of cisplatin is crucial to the clinical validity of our model. Furthermore, rates of AKI are higher in cancer patients than in critically ill patients without cancer. Thus, we utilized an inducible, transgenic mouse model of mutant Kras lung adenocarcinoma in combination with our repeated dosing regimen of cisplatin. Mice with lung adenocarcinoma that received cisplatin had decreased overall survival, earler peaks in kidney injury, and worsened renal fibrosis compared to non cancer mice treated with the cisplatin. Western blot analysis indicated an increase in EGFR protein levels and subsequent differential activation of EGFR-mediated signaling pathways in cancer mice treated with cisplatin. EGFR is highly involved in kidney injury, and is a major contributor to renal fibrosis. Thus, we hypothesized that blocking EGFR activation with erlotinib, an FDA-approved EGFR inhibitor, would protect against the development of renal fibrosis. Combinatorial treatment of both non cancer and cancer with cisplatin + erlotinib caused severe AKI. Thus, erlotinib is not a viable renoprotective agent in our repeated dosing model of cisplatin-induced kidney injury. 
CHAPTER 2

\begin{abstract}
SUB-CLINICAL KIDNEY INJURY INDUCED BY REPEATED CISPLATIN ADMINISTRATION RESULTS IN PROGRESSIVE CHRONIC KIDNEY DISEASE
\end{abstract}

\title{
INTRODUCTION
}

Patients with AKI are ten times more likely to develop CKD than patients without AKI $(25,57)$. This risk further increases with the severity of AKI, as well as having repeated episodes of injury $(1,26,29,58)$. However, whether or not AKI progresses to CKD is still controversial. Many of the same processes involved in adaptive repair after kidney injury can contribute to worsening injury if these processes are sustained for long periods of time. These processes have been termed "mechanisms of maladaptive repair" (Figure 1.1) (1). Most studies of maladaptive repair have been performed with the I/R model of AKI, which focuses more on the vascular injury that occurs with AKI. Therefore, there is a need for a cisplatin model to better understand how these mechanisms of maladaptive repair are important for the progression of cisplatin-induced AKI to CKD.

Our group and others have shown that the repeated administration of low dose cisplatin causes interstitial fibrosis, an endpoint pathology indicative of CKD $(50,51,54-$ $56,59)$. The development of fibrosis in these models is accompanied with little to no cell 
death. Thus, targeting apoptotic and necrotic signaling pathways may be a poor therapeutic option for cisplatin-induced kidney injury (60). Furthermore, these studies highlight that targeting fibrotic signaling pathways may be a better therapeutic option for injury caused by cisplatin. While fibrosis is a hallmark pathology of CKD, CKD is a progressive, multi-faceted disease with many different pathologies including glomerulosclerosis, endothelial dysfunction and damage, and chronic inflammation (1, $38,61)$. For cisplatin-induced kidney injury, there is a lack of knowledge about which mechanisms may contribute to the aforementioned pathologies associated with CKD. Identifying these mechanisms would aid in the development of therapies to target the development and progression of CKD after cisplatin treatment.

We have previously shown that mice develop interstitial fibrosis and are able to survive the entirety of our repeated dosing regimen $(7 \mathrm{mg} / \mathrm{kg}$ cisplatin once a week for 4 weeks) (50). Building upon this work we hypothesized that, due to the progressive nature of CKD, glomerular pathologies and endothelial damage may only be seen at a later time point with our model. To this end, we treated mice with our repeated dosing regimen of cisplatin and examined long-term kidney outcomes at the 6 month time point. To gain insight into components of fibrotic signaling pathways that may be changing early on with repeated cisplatin administration, we also treated mice with only 2 doses of cisplatin and euthanized them at Day 10.

After Dose 2 of cisplatin, there was subtle inflammation, but no overt changes in kidney function, tubular damage, and no interstitial fibrosis. While there was only a subtle change in kidney function after treating mice with 4 doses of cisplatin at Day 24, there was a significant increase in inflammation, endothelial dysfunction, and the 
development of interstitial fibrosis. Six months post cisplatin treatment, inflammation and interstitial fibrosis were worse. At this time point, mice developed glomerular pathologies, and there was development of atherosclerotic lesions in the kidneys as evidenced by increased CD31 expression in the medulla. These data indicate that the nephrotoxic effects of repeated cisplatin dosing are long-lasting, leading to the maintenance of maladaptive repair processes including chronic inflammation, worsened interstitial fibrosis, and glomerular/endothelial changes contributing to decreased kidney function.

\section{MATERIALS AND METHODS}

Animals. For age out studies, 8 week old male FVB/n mice (001800, The Jackson Laboratory, Bar Harbor, ME) were maintained under standard laboratory conditions. All animals were housed under a 12:12 hour light-dark cycle and provided food and water ad libitum. Animal procedures were approved by the Institutional Animal Care and Use Committee (IACUC) at University of Louisville, and followed the appropriate guidelines set forth by the American Veterinary Medical Association. Pharmaceutical grade cisplatin was purchased from the University of Louisville Hospital Pharmacy $(1 \mathrm{mg} / \mathrm{ml}$ in normal saline, Teva Pharmaceuticals). Mice were intraperitoneally injected at 8:00 a.m. with either saline vehicle $\left(0.9 \%\right.$ saline in $\left.\mathrm{ddH}_{2} \mathrm{O}\right)$ or cisplatin $(7 \mathrm{mg} / \mathrm{kg})$ once a week for either two or four doses (Figure 2.1). At Day 10 (3 days after Dose 2), a cohort of saline vehicle and cisplatin treated mice were euthanized. At Day 24 (3 days after Dose 4), a second cohort of saline vehicle and cisplatin treated mice were euthanized. A third cohort of mice received 4 doses of saline vehicle or cisplatin, and were sacrificed 6 months after the final dose. For this experiment, mice were monitored for weight loss or 
high levels of discomfort/stress on a daily basis, and were euthanized if weight loss was greater than $20 \%$.

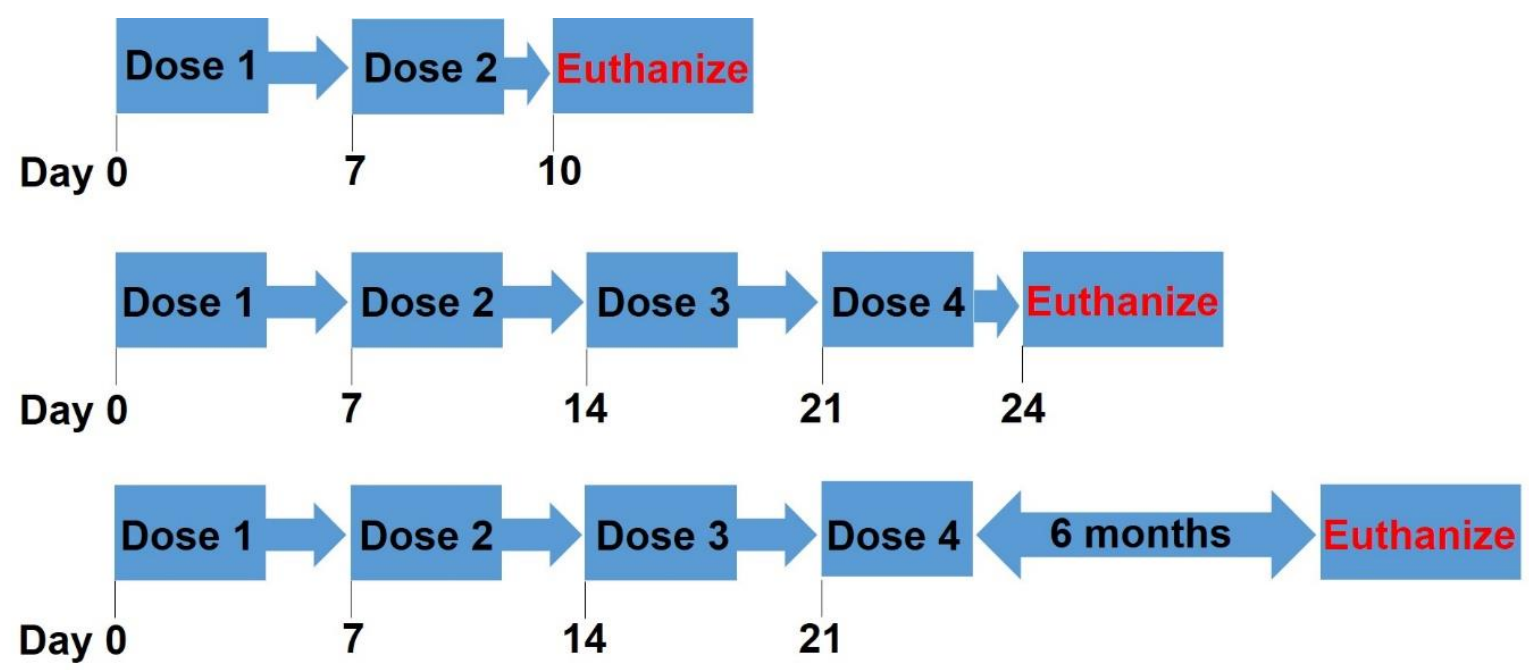

Figure 2.1. Repeated dosing regimen. 8 wk old, male FVB mice were treated with either 2 doses saline vehicle or cisplatin $(7 \mathrm{mg} / \mathrm{kg})$ and euthanized at Day 10. Another group was treated with either 4 doses of saline vehicle or cisplatin $(7 \mathrm{mg} / \mathrm{kg})$ and euthanized at Day 24, or aged out 6 months post-treatment and then euthanized. 
Sample collection and storage. Urine was collected prior to euthanasia and stored at $80^{\circ} \mathrm{C}$. Upon euthanasia, blood was collected and placed on ice. Plasma was then prepared by spinning blood samples at $10,000 \times \mathrm{xg}$ for 10 minutes at $4^{\circ} \mathrm{C}$, and stored at $80^{\circ} \mathrm{C}$. Kidneys were removed, sliced, and tissue was allocated for appropriate use (Figure 2.2). Tissue for protein, RNA, and all extra kidney tissue were flash-frozen in liquid nitrogen, and samples for histology/immunohistochemistry were fixed in 10\% neutral buffered formalin.

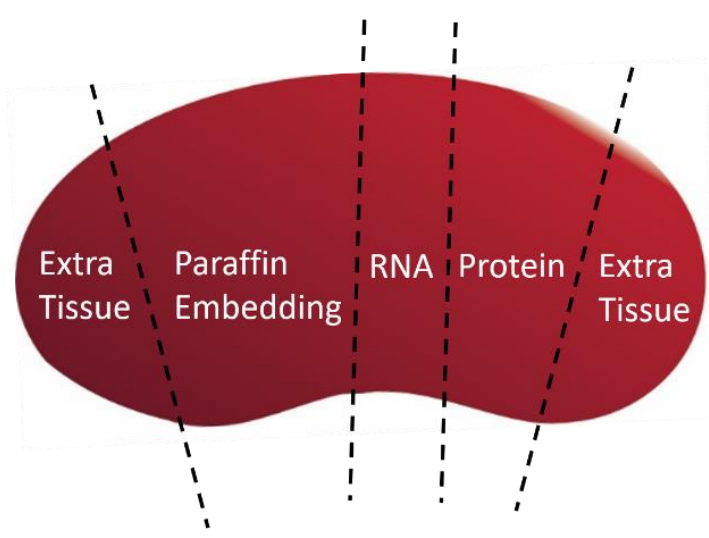

Figure 2.2. Allocation of kidney tissue. Upon euthanasia, the right kidney was removed, and tissue was allocated for protein isolation, RNA isolation, and paraffin embedding as pictured. The left kidney and remaining tissue from the right kidney were stored at $-80^{\circ} \mathrm{C}$.

BUN determination. Blood urea nitrogen (BUN) levels in plasma were determined after Dose 2, 4, and after 6 month age out using a kit from AMS Diagnostics (80146, AMS Diagnostics, Weston, FLA) per the manufacturer's instructions.

ELISAs. ELISAs for neutrophil gelatinase associated lipocalin (NGAL; DY1857, R\&D Systems, Minneapolis, MN) and albuminuria (1011, Exocell, Philadelphia, PA) were 
done on urine samples after Dose 2, 4, and after 6 month age out following manufacturers' protocols.

Gene expression. Total RNA was isolated using the EZNA Total RNA Kit (R6834-02, Omega, Weston, FLA) per manufacturer's protocol. cDNA was made from $1 \mu \mathrm{g}$ of total RNA using High-Capacity Reverse Transcriptase (4368814, Life Technologies, Grand Island, NY) following manufacturer's instructions. Gene-specific cDNA was quantified using real-time QRTPCR and either pre-designed Taqman assays, or self-designed Sybr assays. The following pre-designed primers were used: (Life Technologies, Grand

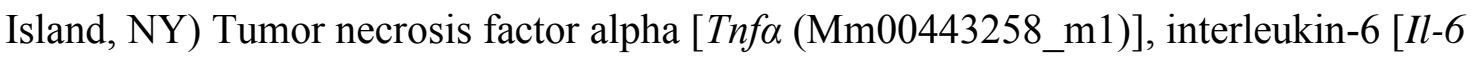
(Mm00446190_m1)], [Cxcl1 (Mm04207460_m1)], monocyte chemoattractant protein-1 [Mcp-1 (Mm00441242_m1)], connective tissue growth factor [ Ctgf (Mm01192932_m1)], collagen subtype 1a1 [Collal (Mm00801666_m1)], arginase-1 [Arg-1 (Mm00475991_m1)] and $\beta_{2}$ microglobulin [B2m (Mm00437762_m1)]. The following gene primers were self-designed: inducible nitrogen oxide synthase (Inos), transforming growth factor beta $1(T g f \beta 1)$, fibronectin $1(F n 1)$, Endothelin-1, TIMP metallopeptidase inhibitor 1 (Timp-1), vascular cell adhesion molecule 1 (Vcam-1), and intercellular adhesion molecule 1 (Icam-1). Primers were used in combination with either iTaq Universal Probes Supermix (172-5134, BioRad, Hercules, CA) or iTaq Universal Sybr Green Supermix (172-5124, BioRad, Hercules, CA).

\section{Preparation of kidney sections for histology and immunohistochemistry. After} fixation in $10 \%$ neutral buffered formalin for 48 hours, kidney samples were stored in $75 \%$ ethanol for at least one hour. Samples were then prepared for paraffin embedding 
(Table 2.1). After embedding, kidney sections were cut 5 microns thick on a microtome, and affixed to slides. Slides were then heated in an oven at $60^{\circ} \mathrm{C}$ for 30 minutes.

\begin{tabular}{|c|c|}
\hline $85 \%$ EtOH & 1 hour, $1 x$ \\
\hline $95 \%$ EtOH & Overnight \\
\hline $100 \%$ EtOH & 1 hour, $3 x$ \\
\hline 100\%EtOH:Histoclear $(1: 1)$ & 30 minutes, $1 x$ \\
\hline Histoclear & 1 hour, $2 x$ \\
\hline Paraplast & 30 minutes, $3 x$ \\
\hline
\end{tabular}

Table 2.1. Preparation of tissue for paraffin embedding. Briefly, samples were run through an ethanol gradient, followed by Histoclear and Paraplast washes before embedding in Paraplast (39502004, McCormick Scientific).

Histology. Kidney sections (5 microns thick) were stained either with Mayer's hematoxylin (MHS32-1L, Sigma) and alcoholic eosin (HT110116, Sigma) (Table 2.2), or Periodic acid-Schiff stain [PAS; (Periodic acid solution: 395132-1L, Sigma) (Schiff's reagent: 3952016-1L, Sigma)] (Table 2.3). Pathological analysis was performed by Dr. Judit Megyesi, a mouse renal pathologist. Measures of both tubular and glomerular damage were evaluated on a scale from 0 to 4 , which ranged from not present $(0)$ to mild (1), moderate (2), severe (3), and very severe (4). 


\begin{tabular}{|c|c|}
\hline Histoclear & 3 minutes, $2 x$ \\
\hline $1: 1$ Histoclear/100\% EtOH & 3 minutes, $1 x$ \\
\hline $100 \%$ EtOH & 3 minutes, $2 x$ \\
\hline $95 \%$ EtOH & 3 minutes, $2 x$ \\
\hline $85 \%$ EtOH & 3 minutes, $1 x$ \\
\hline $75 \%$ EtOH & 3 minutes, $1 x$ \\
\hline Distilled water & 3 minutes, $1 x$ \\
\hline Mayer's Hematoxylin & 15 minutes, $1 x$ \\
\hline Warm running tap water & 15 minutes, $1 x$ \\
\hline Distilled water & 30 seconds, $1 x$ \\
\hline $95 \%$ EtOH & 30 seconds, $1 x$ \\
\hline Alcoholic Eosin & 15 seconds, $1 x$ \\
\hline $95 \%$ EtOH & 3 minutes, $2 x$ \\
\hline 100\% EtOH & 3 minutes, $2 x$ \\
\hline Histoclear & 3 minutes, $2 x$ \\
\hline
\end{tabular}

Table 2.2. Hematoxylin and Eosin staining. Protocol for H\&E staining.

\begin{tabular}{|c|c|}
\hline Histoclear & 3 minutes, $2 x$ \\
\hline $1: 1$ Histoclear $/ 100 \%$ EtOH & 3 minutes, $1 x$ \\
\hline $100 \%$ EtOH & 3 minutes, $2 x$ \\
\hline $95 \%$ EtOH & 3 minutes, $2 x$ \\
\hline $85 \%$ EtOH & 3 minutes, $1 x$ \\
\hline $75 \%$ EtOH & 3 minutes, $1 x$ \\
\hline Distilled water & 3 minutes, $1 x$ \\
\hline Periodic Acid solution & 5 minutes, $1 x$ \\
\hline Distilled water & 30 seconds, $1 x$ \\
\hline Schiff's Reagent & 15 minutes, $1 x$ \\
\hline Running tap water & 5 minutes, $1 x$ \\
\hline Mayer's Hematoxylin & 3 minutes, $1 x$ \\
\hline Running tap water & Until sections turn purple \\
\hline 95\% EtOH & 3 minutes, $2 x$ \\
\hline 100\% EtOH & 3 minutes, $2 x$ \\
\hline Histoclear & 3 minutes, $2 x$ \\
\hline
\end{tabular}

Table 2.3. Periodic acid-Schiff staining. Protocol for PAS staining.

Immunohistochemistry. For alpha smooth muscle actin ( $\alpha \mathrm{SMA})$

immunohistochemistry (IHC), kidney sections (5 microns thick) were rehydrated with

Histoclear (HS-200, National Diagnostics) followed by an ethanol gradient. Sodium

citrate buffer ( $\mathrm{pH}$ 6.0) was used for antigen retrieval. Antigen retrieval was performed in a vegetable steamer for 75 minutes, and slides were cooled at room temperature (RT) for 
60 minutes. Slides were then rinsed in $\mathrm{dH}_{2} \mathrm{O}$ for five minutes. Endogenous peroxidases were blocked with $3 \%$ hydrogen peroxide for 10 minutes, and slides were washed twice in PBS for 5 minutes each. Slides were then blocked for avidin for 10 minutes, washed in PBS for five minutes, and then blocked for biotin for 10 minutes (SP2001, Dako, Burlingame, CA), followed by another PBS wash. Slides were further blocked in 5\% normal goat serum in PBS for 1 hour at RT (PK-7100, Vector Laboratories). Alpha smooth muscle actin primary antibody (ab5694, Abcam) was diluted 1:400 in antibody diluent $[(0.5 \mu \mathrm{g} / \mathrm{ml}), 8112 \mathrm{~L}$, Cell Signaling $]$ and added to sections. Slides were then coverslipped and incubated with the primary antibody overnight at $4^{\circ} \mathrm{C}$. The following morning, slides were rinsed 3 times for 5 minutes each with PBS. Biotinylated goat antirabbit IgG antibody [(1:25000), PK-7100, Vector Laboratories] was added to slides and incubated for 30 minutes at RT. Slides were then rinsed 2 times for 5 minutes in PBS before Vector ABC reagent was added (PK-7100, Vector Laboratories) for 30 minutes at RT. Slides were rinsed two times for 5 minutes with PBS. NovaRed peroxidase substrate solution (SK-4800, Vector Laboratories) was added to slides for seven minutes, and slides were rinsed in $\mathrm{dH}_{2} \mathrm{O}$ for 5 minutes. Slides were counterstained with Modified Mayer's Hematoxylin (72804, Thermo-Scientific) for 30 seconds, and then rinsed with $\mathrm{dH}_{2} \mathrm{O}$ until water ran clear. Bluing solution (1.5\% ammonium hydroxide in $70 \%$ ethanol) was added to slides for 30 seconds, and then rinsed in $\mathrm{dH}_{2} \mathrm{O}$ for 5 minutes. Following this step, slides were dehydrated in the ethanol gradient to Histoclear, fixed with Permount (SP15-100, Fisher), and coverslipped. Images of whole kidney sections were taken using the Aperio Slide Scanner. 
For CD31 IHC, kidney sections were rehydrated as described for $\alpha$ SMA IHC. Sodium citrate buffer $\left(\mathrm{pH}\right.$ 6.0) was used for antigen retrieval for 40 minutes at $95^{\circ} \mathrm{C}$. Slides were serum blocked with goat serum for 2 hours at RT (PK-7100, Vector Laboratories) and washed twice with TBS $0.01 \%$ Triton X-100 for 5 minutes. Slides were then blocked for avidin and biotin as described for $\alpha$ SMA IHC. CD31 antibody (ab124431, Abcam) was added to slides at 1:500 dilution in TBS with 1\% BSA. TBS with $1 \%$ BSA was added to negative controls. Slides were coverslipped and allowed to incubate overnight at $4^{\circ} \mathrm{C}$. The following morning, slides were washed twice with TBS $0.01 \%$ Triton X-100 for 5 minutes. Endogenous peroxidases were blocked with 3\% hydrogen peroxide for 30 minutes, and slides were washed with TBS $0.01 \%$ Triton X100. Biotinylated goat anti-rabbit IgG antibody in TBS with $1 \%$ BSA [(1:25000), PK7100, Vector Laboratories] was added to slides and incubated for 30 minutes at RT. Slides were then rinsed 2 times with TBS $0.01 \%$ Triton X-100 for 5 minutes before Vector $\mathrm{ABC}$ reagent was added (PK-7100, Vector Laboratories) for 30 minutes at room temperature. Slides were then rinsed two times for 5 minutes with TBS $0.01 \%$ Triton X100. NovaRed peroxidase substrate solution (SK-4800, Vector Laboratories) was added to slides for 5 minutes, and then slides were rinsed in $\mathrm{dH}_{2} \mathrm{O}$ for 5 minutes. Slides were counterstained with Modified Mayer's Hematoxylin and processed as described for aSMA IHC.

For F4/80 IHC, kidney sections (5 microns thick) were rehydrated with Histoclear followed by an ethanol gradient. Target retrieval solution (S1699, Dako) was diluted 1:10 in $\mathrm{ddH}_{2} \mathrm{O}$, and slides were incubated in antigen retrieval solution at $72^{\circ} \mathrm{C}$ overnight. The next morning, slides were washed once in TBS for 5 minutes. Slides were then 
blocked with hydrogen peroxide, avidin, and biotin as described above for $\alpha$ SMA IHC, but using TBS for washes. Slides were then washed with TBS $0.01 \%$ Triton X-100 for 5 minutes before blocking with $10 \%$ normal goat serum (31873, Invitrogen) in TBS $0.01 \%$ Triton X-100 for 30 minutes. Primary F4/80 antibody (ab16911, Abcam) was added at a 1:50 dilution in 10\% normal goat serum. Slides were coverslipped and incubated at RT for 2 hours. Slides were then washed with TBS $0.01 \%$ Triton X-100 twice for 5 minutes. Biotinylated rat $2^{\circ}$ antibody (BA-9401, Vector) was added to all sections and incubated for 15 minutes, followed by two washes with TBS for 5 minutes each. ABC reagent (PK6104, Vectastain) was added for 30 minutes, and then slides were washed with TBS twice for 5 minutes each. DAB substrate solution (E885-200ml, Amresco) was added to slides at RT for approximately 10 minutes. Slides were then counterstained, mounted, and imaged as described for $\alpha$ SMA IHC.

Sirius red/ fast green staining for total collagen. Kidney sections (5 microns thick) were rehydrated in Histoclear followed by an ethanol gradient. Slides were then dipped in a Coplin jar containing PBS with $0.1 \%$ Tween 20 (PBST) ten times, and this was followed by a 5 minute wash with PBST. Slides were then washed with distilled water twice for 5 minutes, and incubated in Sirius red/fast green stain $[0.1 \%$ sirius red (sirius $\mathrm{red} /$ direct red 80,365548 , Sigma) and $0.1 \%$ fast green (fast green FCF, F7258, Sigma) in saturated picric acid (5860-32, Ricca Chemicals, Arlington, TX)] for 30 minutes in the dark. Slides were then washed with 5\% glacial acetic acid water until water was clear. Slides were dehydrated through the ethanol gradient, followed by Histoclear before slides were mounted with Permount and coverslipped. Images of whole kidney were taken 
using Aperio Slide Scanner and optical density of Sirius red was quantified using FijiImage $\mathrm{J}$ and the following equation: $\mathrm{OD}=\log$ (maximum intensity/mean intensity).

Statistical analysis. Data are expressed as means \pm SEM for all experiments. Multiple comparisons of normally distributed data sets were analyzed by two way ANOVA, as appropriate, and group means were compared using Tukey posttests. The criteria for statistical differences were: $* \mathrm{P}<0.05, * * \mathrm{P}<0.01,{ }^{* * *} \mathrm{P}<0.001$, and $* * * * \mathrm{P}<0.0001$.

\section{RESULTS}

Kidney injury is detectable after Dose 2 of cisplatin, and kidney function continues to decline 6 months post cisplatin treatment. Mice were treated with either 2 doses or 4 doses of saline or cisplatin $(7 \mathrm{mg} / \mathrm{kg})$ and euthanized 72 hours after the final dose. Another group of mice was treated with 4 doses of saline or cisplatin $(7 \mathrm{mg} / \mathrm{kg})$ and aged for 6 months. BUN and albuminuria are common measures of kidney function, and NGAL is a FDA-approved urinary biomarker for kidney injury $(19,20,62,63)$. BUN was slightly elevated D4 (Dose 4). BUN was significantly increased 6 months post cisplatin treatment (6 mo. post CDDP) (Fig 2.3A). Albuminuria was elevated at D2 and D4 of cisplatin, but returned to baseline 6 mo. post CDDP (Fig 2.3B). NGAL was slightly elevated after Dose 2 (D2), but significantly increased after D4. NGAL levels were not elevated 6 mo. post CDDP (Fig 2.3C). Taken together, these data indicate that mice develop albuminuria after D2, but albuminuria is no longer detectable 6 mo. post CDDP. In comparison, significant changes in BUN do not occur until 6 mo. post CDDP, indicating a progressive and permanent loss of kidney function even when injury, as measured by NGAL, was no longer present. 
(A)

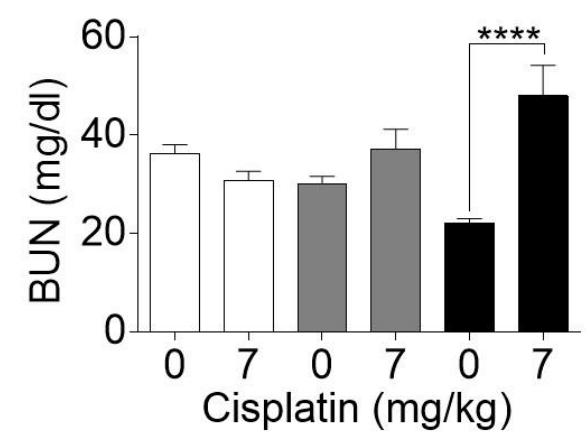

(B)

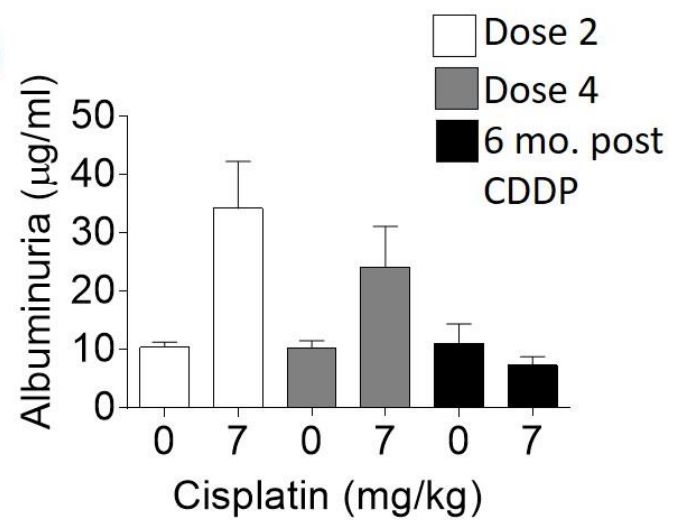

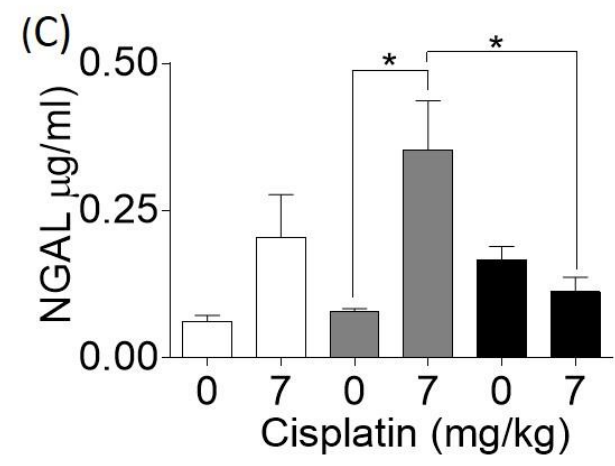

Figure 2.3. Markers of kidney function and injury during and after repeated

cisplatin treatment. 8 wk old FVB mice were treated with either saline vehicle or cisplatin $(7 \mathrm{mg} / \mathrm{kg}$ ) once a week for two weeks and euthanized 3 days after Dose 2. Another group was treated with either saline vehicle or cisplatin $(7 \mathrm{mg} / \mathrm{kg})$ once a week for four weeks and euthanized 3 days after Dose 4, or aged for 6 months following treatment (6 mo. post CDDP). (A) BUN measured in plasma. (B) Albuminuria measured in urine. (C) NGAL measured in urine. Data are expressed as mean $\pm \mathrm{SEM} ; \mathrm{n}=5-10$. Statistical significance was determined by Two-Way ANOVA followed by Tukey post-test. ${ }^{*} \mathrm{P}<0.05,{ }^{* * * *} \mathrm{P}<0.0001$. 


\section{Repeated cisplatin administration causes tubular injury that is still present 6}

months post-treatment. Cisplatin-induced AKI results in proximal tubule cell damage, and we have previously shown that many of the same types of tubular damage occur with repeated cisplatin treatment $(9,10,14,15)$. Tubular dilation was present after D4, and tubules remained dilated 6 mo. post CDDP (Fig 2.4A). There was a slight loss of brush borders after D2, but the severity increased after D4 and damage remained 6 mo. post CDDP (Fig 2.4B). Tubular degeneration occurred after D4 and was still present 6 mo. post CDDP (Fig 2.4C). Severity of tubular cast formation was high after D4 and 6 mo. post CDDP (Fig 2.4D). Interestingly, there was no tubular necrosis with cisplatin treatment (Fig 2.4E). While there was a slight increase in inflammatory cell infiltration after both D2 and D4, there was a significant increase in inflammatory cell infiltration 6 mo. post CDDP (Fig 2.4F). There was also a significant increase in distal tubule damage 6 mo. post CDDP (Fig 2.4G). Taken together, these data indicate that severe tubular injury does not occur until after D4 of cisplatin, and that inflammation and distal damage are progressive. 
(A)

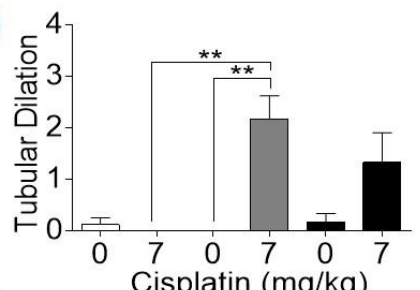

(C)

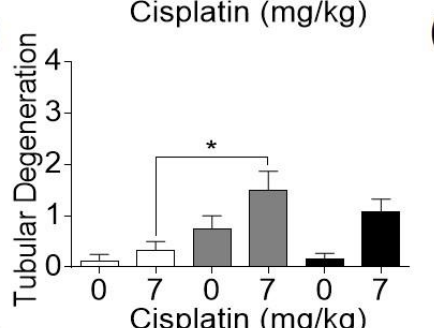

(E)

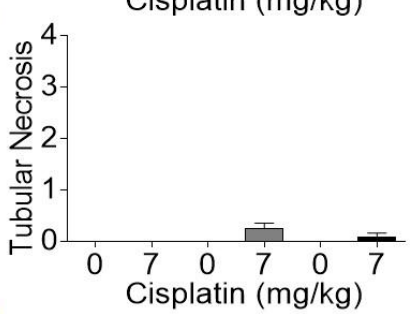

(G)

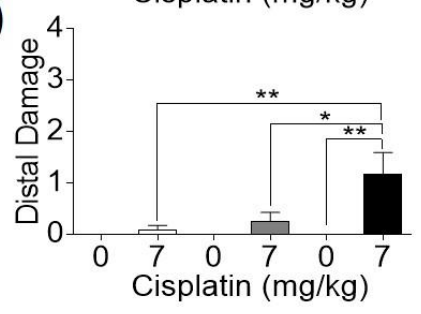

(B)

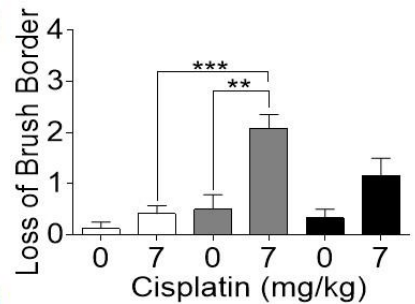

(D)

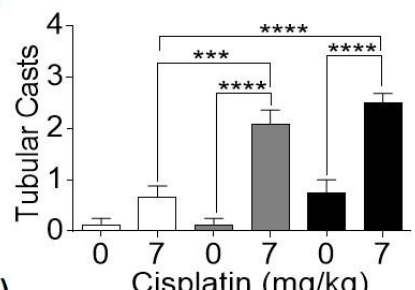

(F)

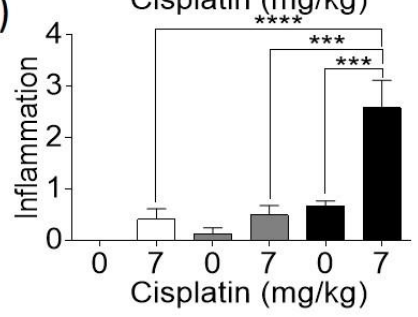

Dose 2

Dose 4

6 mo. post

CDDP

Figure 2.4. Tubular injury during and after repeated cisplatin treatment. $8 \mathrm{wk}$ old FVB mice were treated with either saline vehicle or cisplatin $(7 \mathrm{mg} / \mathrm{kg})$ once a week for two weeks and euthanized 3 days after Dose 2. Another group was treated with either saline vehicle or cisplatin $(7 \mathrm{mg} / \mathrm{kg})$ once a week for four weeks and euthanized 3 days after Dose 4, or allowed to age for 6 months following treatment (6 mo. post CDDP). Severity of (A) tubular dilation, (B) loss of brush border, (C) tubular degeneration, (D) tubular casts, (E) tubular necrosis, (F) inflammation, and (G) distal damage was determined by a renal pathologist. Data are expressed as mean \pm SEM; $n=5-7$. Statistical significance was determined by Two-Way ANOVA followed by Tukey post-test. $* \mathrm{P}<0.05$, ** $\mathrm{P}<0.01$, *** $\mathrm{P}<0.001$, and $* * * * \mathrm{P}<0.0001$. 


\section{Inflammatory cytokine and chemokine levels are altered with repeated cisplatin}

treatment and after 6 month age out. Inflammation is one of the hallmarks of AKI, and chronic inflammation contributes to renal fibrosis $(1,9,10,15,64-66)$. Thus, we wanted to determine levels of disease-related inflammatory cytokines and chemokines. $\mathrm{TNF} \alpha$ is the major mediator of inflammation in cisplatin-induced AKI $(17,67)$. Tnf $\alpha$ mRNA levels were elevated 2-fold after D2, and levels were significantly increased at D4 (Fig 2.5A). Tnf $\alpha$ levels remained significantly elevated 6 mo. post CDDP (Fig 2.5A). Il6 increased at D2 and remained elevated after D4 (Fig 2.5B). 6 mo. post CDDP, Il6 levels increased significantly (Fig 2.5B). Mcp-1, a chemokine for monocyte recruitment, increased slightly after D2, and was elevated 3-fold at D4 and 6 mo. post CDDP (Fig 2.5C). Cxcl1, a chemokine for neutrophil recruitment, increased 5-fold after D2, and was significantly increased at D4 (Fig 2.5D). 6 mo. post CDDP, Cxcll levels were elevated 7-fold (Fig 2.5D). These data indicate that inflammatory cytokine and chemokine levels increase with each dose of cisplatin examined in this study, and remain elevated after cisplatin treatment indicative of chronic inflammation. 
(A)

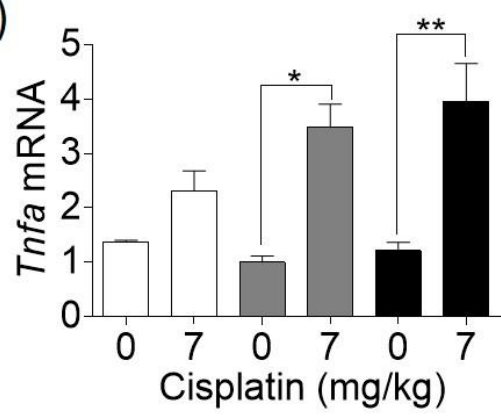

(C)

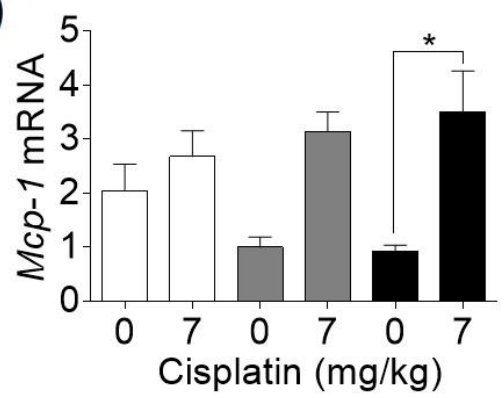

(B)

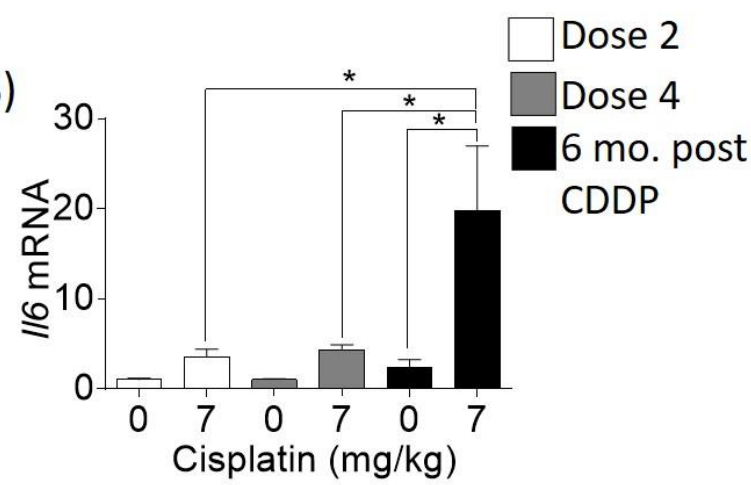

(D)

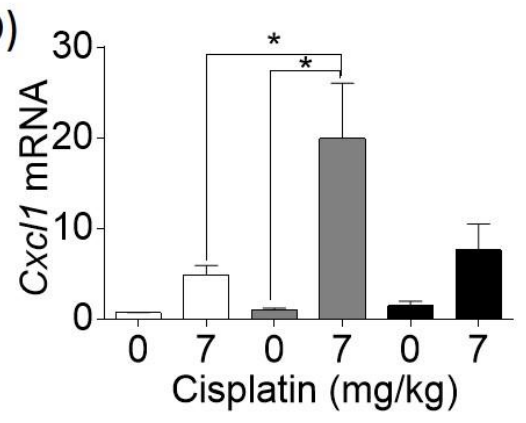

Figure 2.5. Inflammatory cytokine and chemokine levels during and after repeated cisplatin treatment. $8 \mathrm{wk}$ old FVB mice were treated with either saline vehicle or cisplatin $(7 \mathrm{mg} / \mathrm{kg})$ once a week for two weeks and euthanized 3 days after Dose 2. Another group was treated with either saline vehicle or cisplatin (7 $\mathrm{mg} / \mathrm{kg}$ ) once a week for four weeks and euthanized 3 days after Dose 4, or allowed to age for 6 months following treatment (6 mo. post CDDP). Levels of (A) Tnfo, (B) Il6, (C) Mcp-1, and (D) Cxcll were measured in the kidney cortex via real-time QRTPCR. Data are expressed as mean \pm SEM; $n=5-10$. Statistical significance was determined by Two-Way ANOVA followed by Tukey post-test. $* \mathrm{P}<0.05$, $* * \mathrm{P}<0.01$ 


\section{Repeated cisplatin administration causes an increase in M2 macrophages.}

Macrophages mediate kidney injury $(1,10,68,69)$. With AKI, M1 macrophages are proinflammatory, while M2 macrophages are anti-inflammatory, although the roles of these macrophage subtypes are less clearly defined in fibrogenesis and CKD pathogenesis (1, $34,68-70)$. Thus, we wanted to determine the presence of macrophages in our model, as well as subtypes of macrophages. F4/80 IHC for macrophages indicated an increase in total macrophages after D4 (Fig 2.6A). Six mo. post CDDP, more macrophages were present (Fig 2.6A). Inos is a marker of M1 macrophages $(71,72)$. Levels of Inos did not change with cisplatin treatment (Fig 2.6B). Arg-1, a marker of M2 macrophages, increased after D4 (Fig 2.6C) $(71,72)$. Six mo. post CDDP treatment, Arg-1 levels were still elevated (Fig 2.6C). These data indicate an increase in macrophages in the kidney after D4 and 6 mo. post CDDP that have an M2 phenotype. 
(A)
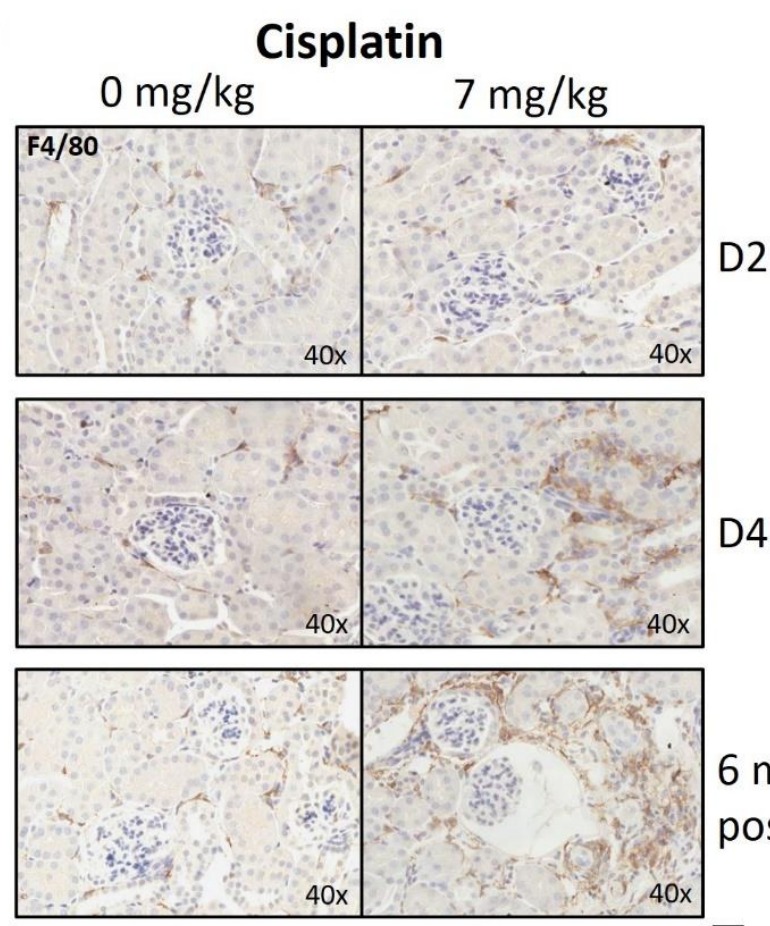

$6 \mathrm{mo}$. post CDDP

(B)

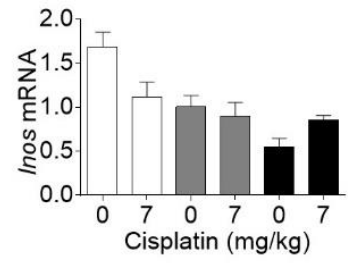

(C) 6 mo. post CDDP

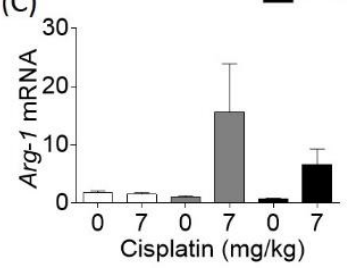

Figure 2.6. Total macrophages and macrophage subtypes during and after repeated cisplatin treatment. 8 wk old FVB mice were treated with either saline vehicle or cisplatin $(7 \mathrm{mg} / \mathrm{kg}$ ) once a week for two weeks and euthanized 3 days after Dose 2. Another group was treated with either saline vehicle or cisplatin $(7 \mathrm{mg} / \mathrm{kg})$ once a week for four weeks and euthanized 3 days after Dose 4, or allowed to age for 6 months following treatment (6 mo. post CDDP). (A) F4/80 IHC staining for total macrophages. Levels of (B) Inos and (C) Arg-1 were measured in the kidney cortex via real-time QRTPCR. Data are expressed as mean \pm SEM; $n=5-10$. 


\section{Repeated cisplatin administration causes chronic pro-fibrotic growth factor}

production. When proximal tubules are injured, they produce growth factors that aid in adaptive repair. However, repeated injury causes proximal tubules to become senescent, leading to the prolonged production of growth factors. Pro-fibrotic factors like TGF $\beta$ and CTGF contribute to the development and maintenance of fibrosis $(1,36,37)$. Tgfbl levels significantly increased after D4 and remained elevated 6 mo. post CDDP (Fig 2.7A). Ctgf increased 2-fold after D4, and remained elevated 6 mo. post CDDP (Fig 2.7B). These data indicate that there is continued production of TGF $\beta$ and CTGF even after repeated cisplatin treatment. 

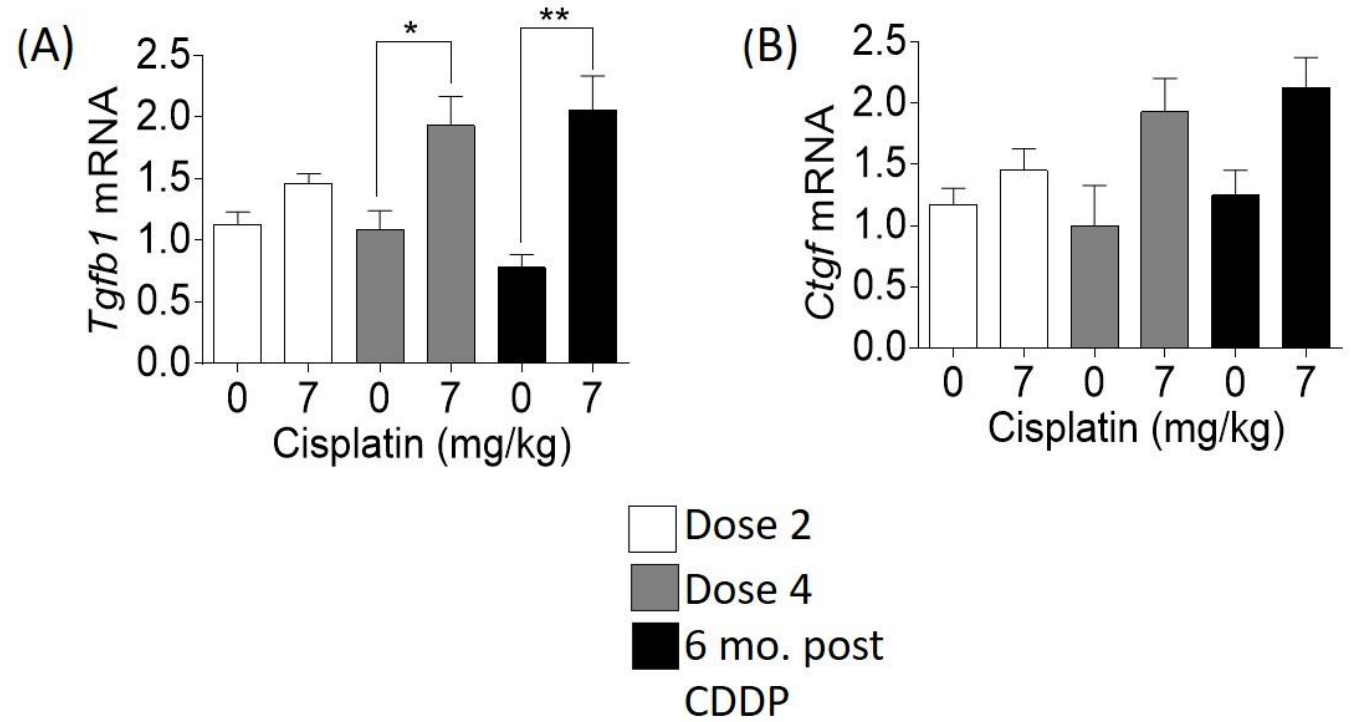

Figure 2.7. Pro-fibrotic growth factor production during and after repeated cisplatin treatment. 8 wk old FVB mice were treated with either saline vehicle or cisplatin $(7 \mathrm{mg} / \mathrm{kg})$ once a week for two weeks and euthanized 3 days after Dose 2. Another group was treated with either saline vehicle or cisplatin $(7 \mathrm{mg} / \mathrm{kg})$ once a week for four weeks and euthanized 3 days after Dose 4, or allowed to age for 6 months following treatment (6 mo. post CDDP). Levels of (A) Tgfbl and (B) Ctgf were measured in the kidney cortex via real-time QRTPCR. Data are expressed as mean \pm SEM; $n=5-10$. Statistical significance was determined by Two-Way ANOVA followed by Tukey post-test. ${ }^{*} \mathrm{P}<0.05, * * \mathrm{P}<0.01$ 


\section{Interstitial fibrosis develops with repeated cisplatin treatment and worsens 6}

months post cisplatin treatment. The development of interstitial fibrosis is a hallmark

of CKD, and fibrosis is progressive $(1,73)$. After D4, interstitial fibrosis was present and severity increased 6 mo. post CDDP (Fig 2.8A). Fibrosis is marked by an increase in both collagen and fibronectin, components of the extracellular matrix (ECM). In addition, myofibroblasts, which deposit collagen, proliferate during fibrogenesis. Myofibroblasts can be detected by aSMA expression, and aSMA IHC indicated an increase in myofibroblasts after D4 (Fig 2.8B) (74). Six mo. post CDDP, there were more $\alpha$ SMA positive myofibroblasts (Fig 2.8B). There was no change in total collagen levels after D2, but there was a significant increase after D4 (Fig 2.8C). Levels of total collagen remained elevated 6 mo. post CDDP (Fig 2.8C). To further confirm the presence of fibrosis, Collal levels were not elevated after D2 of cisplatin, but increased 3-fold after D4 and remained elevated 6 mo. post CDDP (Fig 2.8D). Fibronectin levels increased only after D4, and remained elevated 2-fold 6 mo. post CDDP (Fig 2.8D). Thus, data indicate that fibrosis only develops after four doses of cisplatin, and that fibrosis is progressive. 


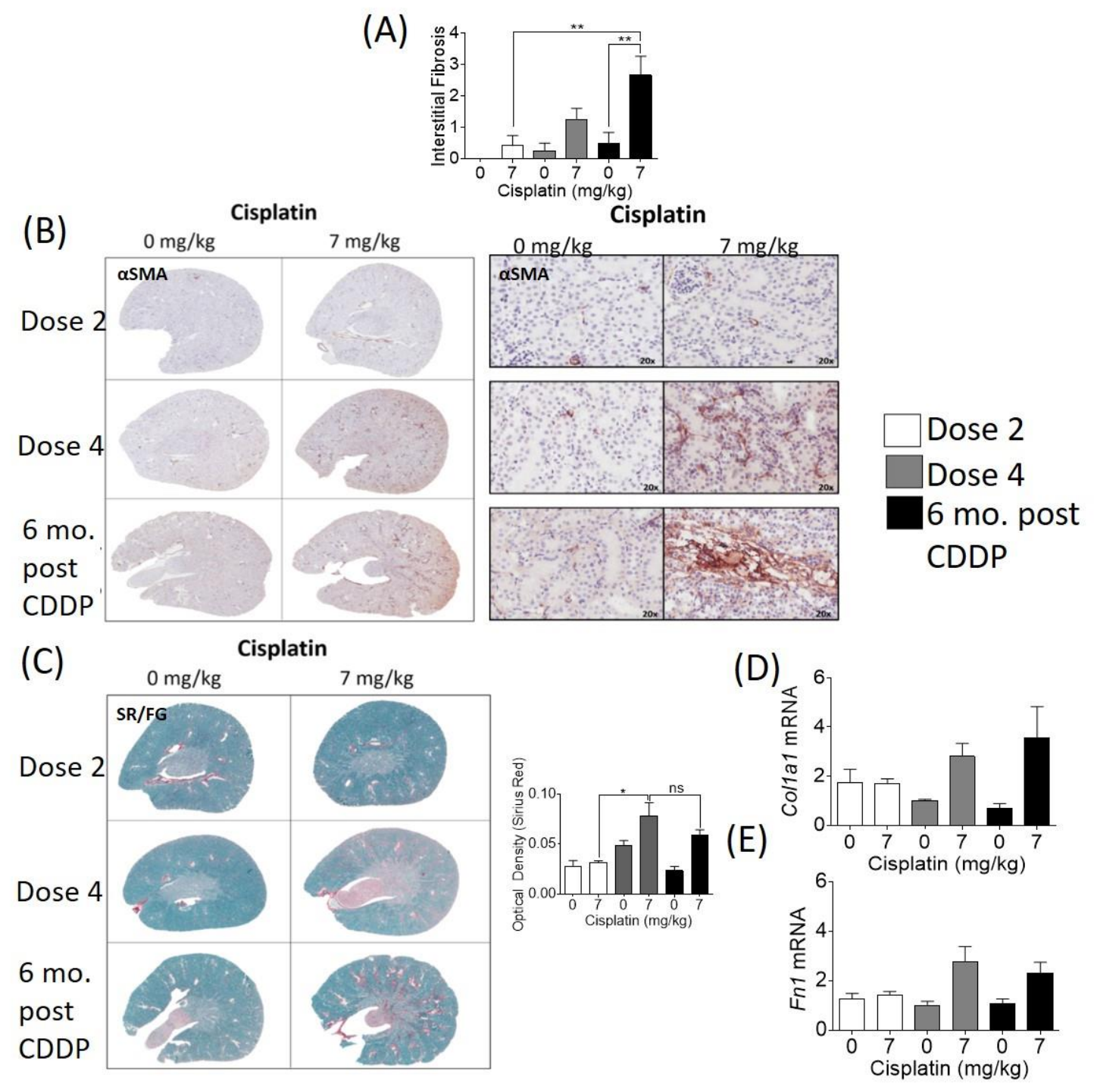




\section{Figure 2.8. Development of interstitial fibrosis during and after repeated}

cisplatin treatment. 8 wk old FVB mice were treated with either saline vehicle or cisplatin $(7 \mathrm{mg} / \mathrm{kg})$ once a week for two weeks and euthanized 3 days after Dose 2. Another group was treated with either saline vehicle or cisplatin $(7 \mathrm{mg} / \mathrm{kg})$ once a week for four weeks and euthanized 3 days after Dose 4, or allowed to age for 6 months following treatment (6 mo. post CDDP). (A) Interstitial fibrosis levels determined by a renal pathologist. (B) Presence of myofibroblasts determined by aSMA IHC. (C) Levels of total collagen determined by SR/FG and optical density quantification of Sirius red staining. Levels of (D) Collal and (E) Fnl measured in kidney cortex via real-time QRTPCR. Data are expressed as mean \pm SEM; $n=5-10$. Statistical significance was determined by two-way ANOVA followed by Tukey posttest. $* \mathrm{P}<0.05, * * \mathrm{P}<0.01$. 
Glomerular damage occurs 6 months post cisplatin treatment. CKD is associated with damage to glomeruli, ultimately resulting in decreased GFR $(38,61,73)$. Renal pathology indicated there was mesangial hypercellularity after D2 and D4 of cisplatin (Fig 2.9A). Interestingly, both saline and cisplatin-treated mice showed increased mesangial hypercellularity in glomeruli 6 mo. post CDDP (Fig 2.9A). Six mo. post CDDP, there was also a significant increase in periglomerular fibrosis and thickening of the glomerular basement membrane (Fig 2.9B,C). Taken together, these data indicate that most glomerular changes occur 6 mo. post CDDP treatment, which may contribute to loss of renal function. 


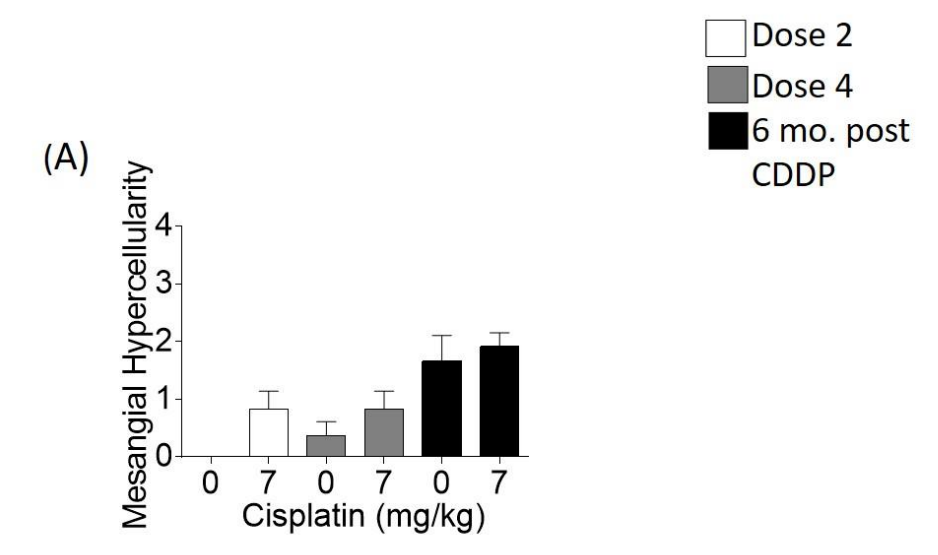

(B)

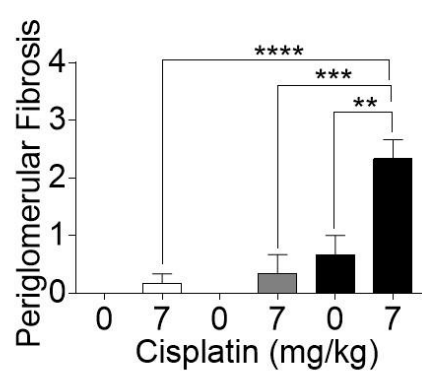

(C)

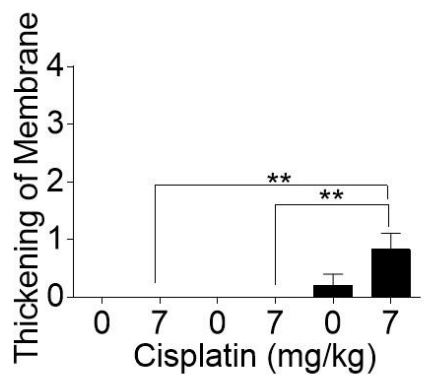

Figure 2.9. Indices of glomerular damage during and after repeated cisplatin

treatment. 8 wk old FVB mice were treated with either saline vehicle or cisplatin (7 $\mathrm{mg} / \mathrm{kg}$ ) once a week for two weeks and euthanized 3 days after dose 2. Another group was treated with either saline vehicle or cisplatin $(7 \mathrm{mg} / \mathrm{kg})$ once a week for four weeks and euthanized 3 days after dose 4, or allowed to age for 6 months following treatment (6 mo. post CDDP). Severity of (A) mesangial hypercellularity, (B) periglomerular fibrosis, and (C) thickening of basement membrane was determined by a renal pathologist. Data are expressed as mean \pm SEM; $n=5-7$. Statistical significance was determined by two-way ANOVA followed by Tukey post-test. $* \mathrm{P}<0.05, * * \mathrm{P}<0.01$. 


\section{Repeated administration of cisplatin causes endothelial dysfunction that is}

maintained 6 months post-treatment. Endothelial dysfunction and subsequent endothelial cell damage are major components of CKD. Endothelial dysfunction is marked by increased production of vasoconstrictors $(3,12)$. During endothelial dysfunction, inflammatory processes are dysregulated, and levels of adhesion molecules are elevated to promote adhesion of leukocytes to endothelial surfaces. Endothelin-1 is a potent vasoconstrictor involved in the regulation of endothelial cells that increases during early endothelial dysfunction (75-78). Endothelin-1 significantly increased after D4, and remained elevated 6 mo. post CDDP (Fig 2.10A). Vcam-1 and Icam-1 levels were slightly elevated after D2, but significantly increased after D4 (Fig 2.10B,C). Levels of Vcam- 1 and Icam- 1 remained slightly elevated 6 mo. post CDDP (Fig 2.10B,C). In addition, Timp- 1 levels increased after D4 and remained elevated 6 mo. post CDDP (Fig 2.10D).

CD31 is highly expressed in endothelial cells, and is used as a marker of angiogenesis during atherosclerotic processes (79). CD31 IHC staining revealed no detectable changes in CD31 in the cortex or medulla after D2 or D4 (Fig 2.10E). However, there was an increase in CD31 staining in the medulla 6 mo. post CDDP (Fig 2.10E). These data indicate that endothelial dysfunction occurs after D2 of cisplatin, worsens after D4, and persists 6 mo. post CDDP. In addition, increased CD31 positivity 6 mo. post CDDP indicates potential angiogenesis associated with the development of atherosclerosis. 
(A)

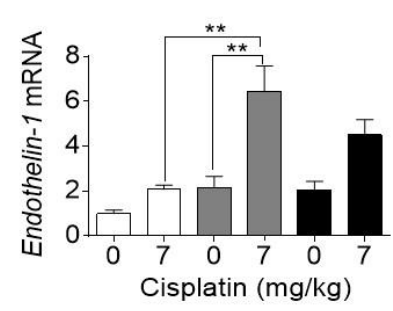

(B)

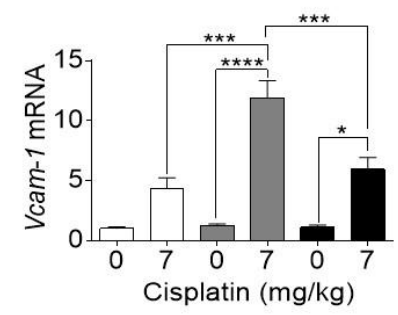

(C)

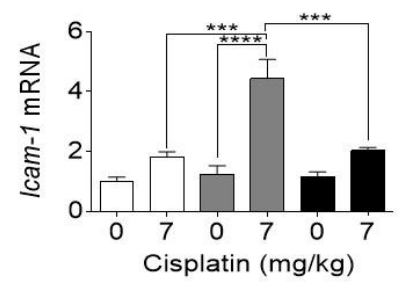

(D)

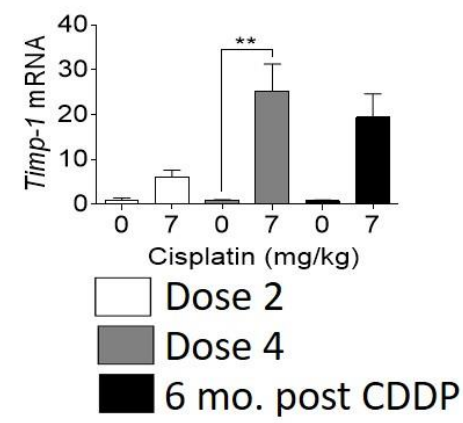

(E)

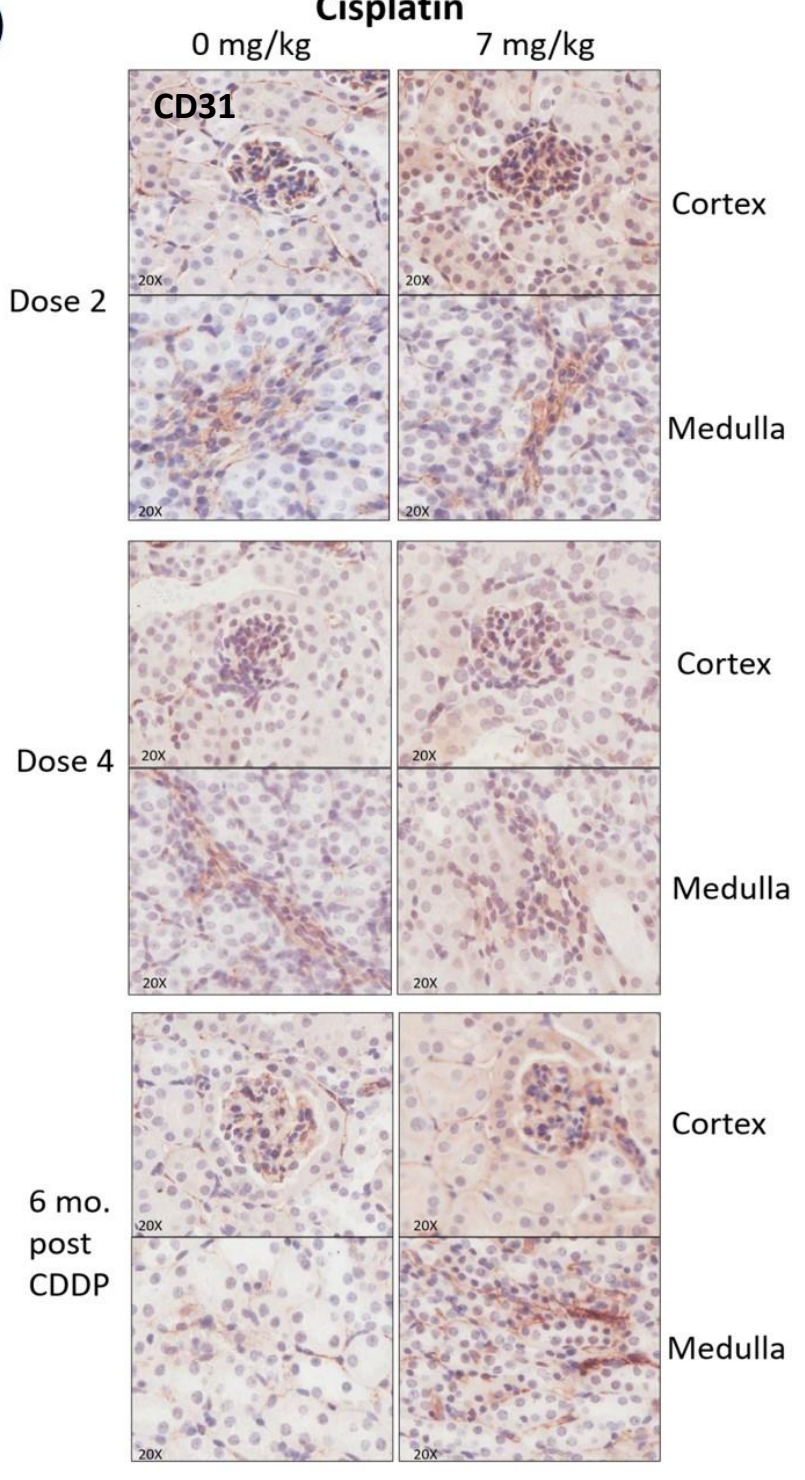


Figure 2.10. Markers of endothelial dysfunction and damage during and after repeated cisplatin treatment. $8 \mathrm{wk}$ old FVB mice were treated with either saline vehicle or cisplatin $(7 \mathrm{mg} / \mathrm{kg}$ ) once a week for two weeks and euthanized 3 days after

Dose 2. Another group was treated with either saline vehicle or cisplatin $(7 \mathrm{mg} / \mathrm{kg})$ once a week for four weeks and euthanized 3 days after Dose 4 or allowed to age for 6 months following treatment (6 mo. post CDDP). Levels of (A) Endothelin-1, (B) Vcam-1, (C) Icam-1, and (D) Timp-1 were measured in kidney via QRTPCR. (E) CD31 IHC stain for endothelial cell damage in cortex and medulla. Data are expressed as mean $\pm \mathrm{SEM} ; \mathrm{n}=5-10$. Data are expressed as mean \pm SEM; $\mathrm{n}=5-10$. Statistical significance was determined by two-way ANOVA followed by Tukey posttest. $* \mathrm{P}<0.05, * * \mathrm{P}<0.01, * * * \mathrm{P}<0.001$, and $* * * * \mathrm{P}<0.0001$ 


\section{DISCUSSION}

By 2020, there will be 15 million new cases of cancer per year worldwide (80). Approximately one fifth of patients will receive cisplatin as part of their therapeutic regimen. While it is well established that patients with AKI are at greater risk for developing CKD, the novel finding of our study is that mild injury caused by repeated low dosing of cisplatin, that does not meet the clinical criteria for AKI, can progress to CKD. Clinically, there is no stringent definition for AKI, but commonly used RIFLE criteria define $\mathrm{AKI}$ as a doubling in $\mathrm{SCr}$, and $30 \%$ of patients that receive cisplatin will develop AKI according to this definition $(19,81,82)$. However, little is known as to whether the $70 \%$ who do not develop clinical AKI are also at risk for poor, long-term outcomes including increased mortality risk, development of CKD, or development of end stage renal disease (ESRD) (24-26, 83). In meta-analyses by Chertow et al. and Coca et al., some cohorts included in these studies defined AKI as a $25 \%$ increase in $\mathrm{SCr}$ $(25,83)$. Even with this mildest form of AKI, there was a $70 \%$ increase in mortality risk $(25,84)$.

$\mathrm{SCr}$ is a poor marker for defining AKI, and does not accurately reflect true renal function as measures are affected by age, gender, protein intake, and drug metabolism (85). Furthermore, changes in $\mathrm{SCr}$ do not occur until there is $50 \%$ or greater loss of nephrons (86). Our model supports the notion that even without meeting the clinical definition of AKI, kidney injury does occur and it leads to poor long-term outcomes. After two doses of cisplatin, we saw tubular pathologies indicative of acute tubular injury, although there was no change in BUN levels. However, there was a 26\% increase in BUN after Dose 4 of cisplatin, and tubular dilation, loss of brush border, tubular 
degeneration, and tubular cast formation were more severe-indicating a mild AKI phenotype. Having ways to detect kidney injury before SCr changes is a clinical challenge and there are few biomarkers available for accurately measuring kidney damage. We believe our model presents the novel opportunity to identify pathologies and their contributing mechanisms that occur early on with cisplatin-induced kidney injury, and in doing so, will aid in the identification of more reliable and accurate biomarkers for both $\mathrm{AKI}$ and $\mathrm{CKD}$. In turn, this may provide clearer, more stringent endpoints for clinical trial design.

As previously discussed, clinically relevant repeated, low dose cisplatin in vivo models have been developed $(50,51,54-56)$. However, our study is the first to look at long-term effects post cisplatin treatment and to show that bona fide CKD can result from repeated cisplatin administration. $\mathrm{FVB} / \mathrm{n}$ mice treated with cisplatin have worsened chronic inflammation, and progressive interstitial fibrosis in the kidney 6 months posttreatment (Table 2.4). These mice also had endothelial dysfunction and glomerular pathologies secondary to tubular injury. This ultimately culminated in a significant decline in kidney function, indicative of CKD.

Of interest, while total inflammation increased 6 months post cisplatin treatment, the only cytokine to increase in this same manner was IL6. IL6 is expressed by podocytes, tubular epithelial cells, and mesangial cells under normal physiological conditions (76). IL6 enhances the response of injured tubular cells to pro-fibrotic stimuli (87). Furthermore, high circulating levels of IL6 are commonly found in ESRD patient, and Zhang et al. have also shown that IL6 levels increase in the kidneys of patients with CKD (88). IL6 activation can also lead to the expression of fibrotic genes and 
endothelin-1, indicating the role of IL6 as a major driver of CKD (88). In addition, pathological levels of IL6 are highly associated with increased macrophages in the kidney, as IL6 is responsible for switching dendritic cells to a macrophage phenotype (76). In our model, $I l 6$ mRNA levels in the kidney were highest in mice 6 months post cisplatin treatment, and this correlated with high $\mathrm{F} 4 / 80$ positive staining for macrophages. The robust increase in $I l 6$ levels at this time point may be indicative of progressive CKD. This provides evidence that IL6 may be a potential therapeutic target for preventing the development and progression of CKD after cisplatin-induced kidney injury, as inhibition or depletion would affect a number of contributing pathways. Indeed, in a model of acute peritoneal inflammation, IL6 $6^{-/-}$mice did not develop fibrosis (64).

An important finding of this study was that total macrophages increased after 4 doses of cisplatin, and more macrophages were detected 6 months post cisplatin treatment. Macrophages are key mediators of AKI, and the prevalence of M1 or M2 macrophages is an indicator of whether adaptive or maladaptive repair is occurring ( 1 , 34, 68, 69). Briefly, M1 macrophages are pro-inflammatory and promote renal injury, whereas M2 macrophages are anti-inflammatory and are essential for injury repair (1). Based on the roles of these subtypes, we would have expected M1 (iNOS expressing) macrophages to be prominent with repeated cisplatin dosing. However, we found that Inos levels did not change with cisplatin treatment. There was a peak in Arg-1 expression (M2 macrophages) after Dose 4, and Arg-1 levels were still elevated 6 months post cisplatin treatment. Thus, macrophages present in the kidney after injury are M2 in nature. This is poignant as the kidney has little to no Arg-1 expressed normally, and increases in $\mathrm{Arg}-1$ can be specifically traced to M2 macrophages $(89,90)$. 
Previous studies have indicated that M2 macrophages are reparative when there is short-term inflammation. In our model chronic, progressive inflammation occurs, indicating M2 macrophages may be contributing to fibrosis with this type of injury. This finding is not surprising as macrophages are known to secrete chemokines involved in the recruitment of fibroblasts, and can activate both resident and recruited myofibroblasts at the site of injury (70). In an I/R study by Kim et al., there was a significant increase in F4/80+ macrophages during the recovery phase after I/R injury, but total number of macrophages continued to increase even after the recovery phase, suggesting their potential role in fibrotic injury (70). There was a shift from M1 to M2 macrophages after the recovery phase, further highlighting the contribution of M2 subtype macrophages in fibrosis (70). To validate the role of M2 macrophages and their contribution to fibrosis, Kim et al. depleted macrophages with liposome clodronate, which improved fibrosis (70). Adoptive transfer studies with both M1 and M2 macrophages indicated that the fibrotic phenotype was only restored with M2 macrophages (70). Our findings with our repeated cisplatin dosing model also indicate the importance of macrophages in fibrogenesis, and that specifically M2 macrophages are contributing to the progression of fibrosis.

While chronic inflammation and M2 macrophages can drive the development of renal fibrosis, this process is also largely mediated by TGF $\beta$. Tgfbl levels significantly increased in the kidney after Dose 4 and remained elevated 6 months post cisplatin treatment. TGF $\beta$ and CTGF are derived from senescent tubular cells that have undergone cell cycle arrest upon injury $(1,36,37)$. These pro-fibrotic growth factors can stimulate proliferation and differentiation of fibroblasts to myofibroblasts (1). $\alpha \mathrm{SMA}+$ 
myofibroblasts increased after Dose 4 and further increased 6 months post cisplatin treatment. Myofibroblasts contribute to accumulation of collagen as evidenced by SR/FG staining, indicative of interstitial fibrosis.

Interstitial fibrosis was detectable after Dose 4, and pathology scoring for interstitial fibrosis indicated increased severity 6 months post cisplatin treatment. Surprisingly, classical markers of interstitial fibrosis such as TGF $\beta$, collagen levels, and fibronectin did not further increase. However, periglomerular fibrosis developed 6 months post cisplatin treatment therefore contributing to the overall presence of fibrosis, and highlighting the development of other glomerular pathologies with this model. The development of interstitial fibrosis and glomerular pathologies after cisplatin treatment recapitulates outcomes in a porcine model of cisplatin treatment (91). Mature, large white female pigs developed interstitial fibrosis in the deep cortex and medulla, and developed glomeruli with thickened Bowman's capsules and capillary changes 24 weeks after intravaneous injection of low dose cisplatin $(91,92)$. We saw a similar interstitial fibrosis pattern in the kidneys of mice with repeated cisplatin treatment after 6 month age-out, as well as glomerular changes at this time point. Porcine kidneys are similar to human kidneys in structure, and have the ability to develop renal fibrosis and glomerular changes that are similar to what is seen with human CKD progression $(93,94)$. However, renal studies with pigs are still relatively new, and mechanistic studies are difficult because genetically manipulated porcine models are still limited (93). Since we are able to recapitulate a similar fibrotic phenotype with our mouse model, this further suggests that our repeated dosing regimen is a clinically relevant model of cisplatin 
nephrotoxicity. In addition, because this model utilizes mice, mechanistic studies are easier and more cost effective.

The development of interstitial fibrosis is often preceded by endothelial dysfunction, a type of injury that occurs early in cisplatin-induced AKI. Endothelial dysfunction also occurs in CKD and diabetes mellitus $(77,78)$. Early dysfunction is marked by an increase in vasoconstrictors and adhesion molecules (78). Endothelin-1 is a sensitive marker of endothelial perturbations, and increases in this vasoconstrictor leads to an increase in arterial stiffness, which if prolonged, could contribute to the development of atherosclerosis (77-79). ICAM-1 and VCAM-1 promote inflammation by allowing leukocytes to adhere to endothelium (78). This process is also promoted by MCP-1 (78). With our model, slight changes in endothelin-1, VCAM-1, and ICAM-1 can be seen after Dose 2 with our repeated cisplatin dosing regimen, these levels are further elevated after Dose 4, and remain slightly elevated 6 months post cisplatin, indicating persistent endothelial dysfunction (79). Furthermore, CD31 expression is often lost in damaged endothelial cells. CD31 IHC in the cortex revealed no overt changes to the endothelium, indicating endothelial dysfunction was occurring without causing actual damage to endothelial cells. However, the role of CD31 in endothelial damage is complex. Aside from endothelial cells, CD31 is highly expressed in leukocytes and tubule cells. CD31 IHC in the medulla showed highly expressing CD31 cells. While further verification needs to be done to determine what these cells are, an increase in CD31 in this context is associated with the development of atherosclerotic lesions (79). Atherosclerosis is another pathology associated with CKD, further 
supporting the notion that repeated cisplatin administration leads to the development of progressive CKD.

In conclusion, our data indicate the effects of repeated treatment with cisplatin, which better mimics the clinical dosing regimen, leads to adverse long-term effects without overt AKI. Specifically, these effects include chronic inflammation, worsened interstitial fibrosis, endothelial dysfunction, declined kidney function, and glomerular pathologies associated with CKD. This model is ideal for both identifying biomarkers for early injury detection, as well as potential targets to halt the development of CKD after cisplatin treatment. This model also provides a clinically relevant model for testing current, potential kidney injury therapeutics that are being developed, with the ultimate goal of finding a potent therapeutic option for cisplatin-induced kidney injury. 


\section{CHAPTER 3}

\section{AGING DOES NOT LEAD TO EXACERBATED AKI OR FIBROSIS}

\section{INTRODUCTION}

In the United States, it is estimated that the elderly population will account for $21 \%$ of the total population by 2030 . In the context of AKI, advanced age is a major health concern. The average age of an in-hospital patient with AKI is 75 years old, and the incidence of AKI increases with each decade of life (1). Experimental data in mice have shown that aging is associated with chronic inflammation, increased oxidative stress, and mitochondrial dysfunction. These physiological changes that occur with aging may contribute to increased susceptibility to AKI or cause exacerbated injury, ultimately resulting in a progressive loss of kidney function. In particular, creatinine clearance begins to decrease after age 30 in humans, and many older people develop glomerulosclerosis, leading to a decline in GFR (95).

The median age for all cancer diagnoses is 65 , and most cancer diagnoses are made in patients over age 55 . Thus, this age group is more likely to be affected by cisplatin nephrotoxicity, and studying the effects of aging in cisplatin-induced kidney injury is important (52). However, it is difficult to draw conclusions about the true risk of cisplatin-induced kidney injury in the elderly population because many patients have comorbidities that make them more susceptible to kidney injury even without cisplatin treatment, particularly cardiovascular disease and diabetes(24). Thus, conclusions about 
increased risk of cisplatin-induced AKI are limited. Furthermore, experimental AKI aging studies often utilize mice at the end of their lifespan (18-24 months old) which do not represent the age group of patients that are often diagnosed with cancer and are most likely to receive cisplatin treatment for their malignancy. In addition, these mouse studies often focus on I/R injury, which focuses mainly on the vascular injury that occurs in AKI rather than the multi-faceted injury that is seen with cisplatin treatment. To improve on previous studies of cisplatin-induced AKI and aging, we utilized 40 week old $\mathrm{FVB} / \mathrm{n}$ mice. These mice better represent a middle-aged population who would be healthy enough to receive cisplatin. We hypothesized that these mice, when treated with one, high dose of cisplatin $(25 \mathrm{mg} / \mathrm{kg})$, would have worsened AKI compared to 8 week old mice treated with the same dose.

Aging is also associated with an increase in cellular senescence that pre-disposes the normal, aging kidney to the development of fibrosis $(1,95)$. Previously, we have developed a more clinically relevant cisplatin dosing regimen that causes fibrosis and CKD (50). Using this repeated dosing regimen, we wanted to determine whether repeated dosing of cisplatin in aged mice would result in worsened fibrosis. To gain insight into this question, a cohort of 8 and 40 week old mice were treated with either saline or $7 \mathrm{mg} / \mathrm{kg}$ cisplatin once a week for four weeks and euthanized at Day 24 .

Data from these studies indicate that aged mice did not have worsened AKI compared to young mice. However, 40 week old mice had basally elevated myofibroblasts and higher total collagen deposition compared to 8 week old mice. Administration of repeated, low dose cisplatin slightly elevated collagen deposition, but there was no difference in overall collagen deposition between cisplatin treated 8 week 
and 40 week old mice. Furthermore, aged mice treated with cisplatin had less of an inflammatory cytokine response compared to young mice. The immune system plays an important role in kidney pathology, and chronic immune cell infiltration is heavily involved in the development of fibrosis $(1,38)$. Immunohistochemistry staining for $\mathrm{T}$ cells and macrophages indicated there were more immune cells in the kidney with cisplatin treatment in 40 week old mice without overt fibrosis. Taken together, our data indicate that aging is not associated with worsened AKI or fibrosis with cisplatin treatment.

\section{MATERIALS AND METHODS}

Animals. Eight and 40 week old, male FVB/n mice were bred in house and provided graciously by Dr. Levi Beverly. Mice were maintained under standard laboratory conditions as previously described in Chapter 2. One cohort of both 8 and 40 week old mice were intraperitoneally injected at 8:00 a.m. with either one dose saline vehicle $\left(0.9 \%\right.$ saline in $\left.\mathrm{ddH}_{2} \mathrm{O}\right)$ or cisplatin $(25 \mathrm{mg} / \mathrm{kg})$, and mice were sacrificed 72 hours after treatment (standard dosing regimen) [Fig 3.1]. Another cohort of mice was intraperitoneally injected at 8:00 a.m. with either saline vehicle $\left(0.9 \%\right.$ saline in $\left.\mathrm{ddH}_{2} \mathrm{O}\right)$ or cisplatin $(7 \mathrm{mg} / \mathrm{kg}$ ) once a week for four weeks and euthanized at Day 24 (repeated dosing regimen) [Fig 3.2]. Mice were monitored for weight loss or high levels of discomfort/stress on a daily basis, and were euthanized if weight loss was greater than $20 \%$. 


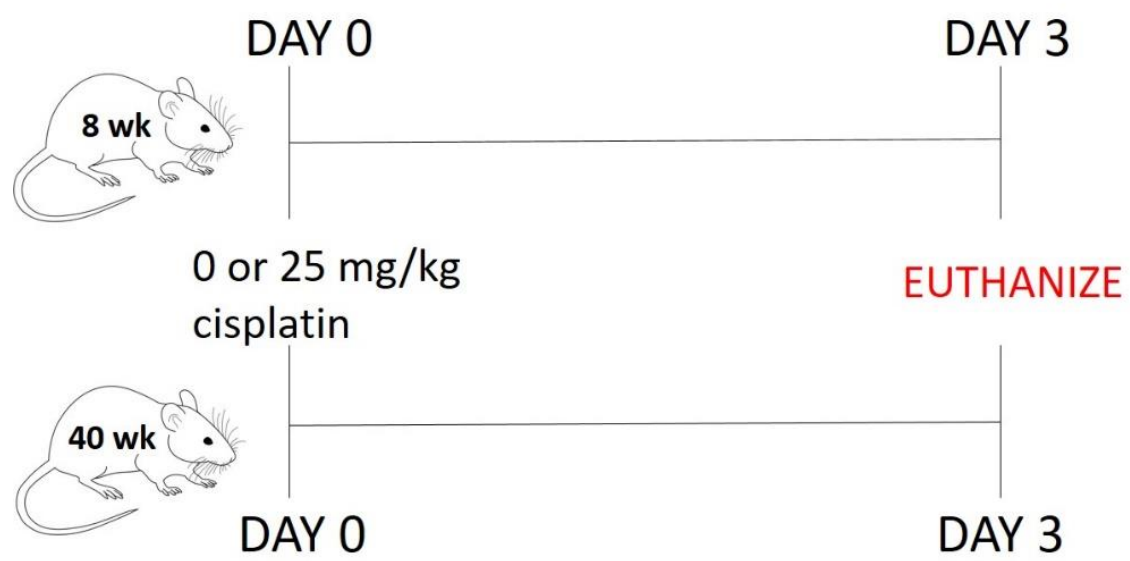

Figure 3.1. Standard dosing regimen of cisplatin. 8 and 40 week old male FVB mice were treated with either saline vehicle $(0 \mathrm{mg} / \mathrm{kg})$ or $25 \mathrm{mg} / \mathrm{kg}$ cisplatin once and euthanized 3 days (72 hours) after initial dosing.

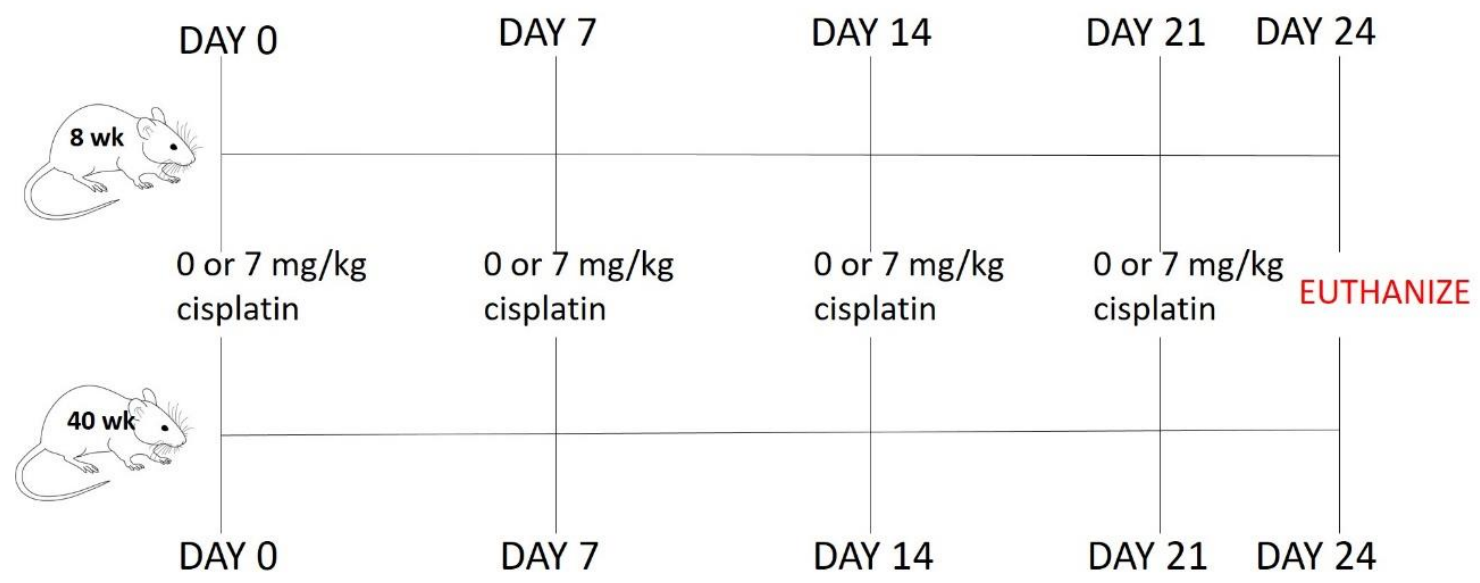

Figure 3.2. Repeated dosing regimen of cisplatin. 8 and 40 week old male FVB mice were treated with either saline vehicle $(0 \mathrm{mg} / \mathrm{kg})$ or $7 \mathrm{mg} / \mathrm{kg}$ cisplatin once a week for four weeks, and euthanized on Day 24.

Antibodies. The following antibodies were purchased from Cell Signaling (Beverly, MA) unless otherwise noted: cleaved caspase-3 (no. 9664), CCAAT/enhancer-binding protein homologous protein (CHOP; no. 2895), transforming growth factor (TGF)- $\beta$ (no. 
3712S), fibronectin (F3648, Sigma-Aldrich, St. Louis, MO), and tubulin (SC-23948, Santa Cruz Biotechnology, Dallas, TX).

Sample collection and storage. Urine was collected and stored at $-80^{\circ} \mathrm{C}$. Blood was collected, and plasma and kidney samples were prepared as previously described in Chapter 2.

BUN determination. Blood urea nitrogen (BUN) levels were determined in plasma using a kit from AMS Diagnostics (80146, AMS Diagnostics, Weston, FLA) per the manufacturer's instructions.

ELISAs. ELISA for neutrophil gelatinase associated lipocalin (NGAL; DY1857, R\&D Systems, Minneapolis, MN) was performed on urine samples using the manufacturers' protocols.

Gene expression. Total RNA was isolated and cDNA was made as outlined in Chapter 2. Gene-specific cDNA was quantified using real-time QRTPCR and either pre-designed Taqman assays, or self-designed Sybr assays. The following pre-designed primers were used: (Life Technologies, Grand Island, NY) Tnf $\alpha$ (Mm00443258_m1), Il6 (Mm00446190_m1), Cxcl1 (Mm04207460_m1), Mcp-1 (Mm00441242_m1), Arg-1 (Mm00475991_m1) and B2m (Mm00437762_m1). Gene primers for Inos and kidney injury molecule-1 (Kim-1) were self-designed. Primers were used in combination with either iTaq Universal Probes Supermix (172-5134, BioRad, Hercules, CA) or iTaq Universal Sybr Green Supermix (172-5124, BioRad, Hercules, CA).

Protein Isolation/Quantification and Western Blot Analysis. Protein was isolated and quantified as previously published (50). Western blot analysis was performed as 
previously published, using 1:5000 dilutions for primary antibodies and 1:40000 dilutions for secondary antibodies. Proteins of interest were detected by chemiluminiscence substrate.

Immunohistochemistry. IHC stainings for $\alpha \mathrm{SMA}$ and F4/80 were performed as previously described (Chapter 2 Materials and Methods). For CD3, deparaffinization, antigen retrieval, and avidin/biotin blocking steps were performed as previously described for $\alpha$ SMA (Chapter 2 Materials and Methods). After avidin/biotin blocking, slides were blocked with 5\% normal goat serum in PBS (Invitrogen 31873) for one hour at room temperature. Rat-anti-human CD3 (BioRad MCA1477) was diluted 1:100 in normal goat serum and applied to tissue sections. Slides were incubated in primary antibody overnight at $4{ }^{\circ} \mathrm{C}$. The next morning, slides were washed three times with PBS for 5 minutes each. Goat-anti-rat biotinylated secondary antibody was diluted 1:250 in PBS and applied to slides for a one hour incubation. During this time $\mathrm{ABC}$ reagent (Vectastain PK-4001) was prepared. After incubation in secondary, slides were washed twice for five minutes in PBS. ABC reagent was then applied to slides for thirty minutes, and slides were processed as previously described for aSMA IHC (Chapter 2 Materials and Methods).

\section{RESULTS}

\section{Overall survival and weight loss in 8 and 40 week old mice treated with the standard} dosing regimen of cisplatin. AKI is associated with a high rate of mortality, ranging from $20-80 \%$. We hypothesized that 40 week old mice (aged mice) treated with high dose cisplatin would have decreased survival, and greater weight loss compared to 8 week old mice (young mice) treated with cisplatin. Young mice that received either 0 
$\mathrm{mg} / \mathrm{kg}$ or $25 \mathrm{mg} / \mathrm{kg}$ cisplatin survived to Day 3, at which point the young, cisplatintreated cohort had an average weight loss of 17.4\% (Fig 3.3A,B). While there was 100\% survival in aged mice that received $0 \mathrm{mg} / \mathrm{kg}$ cisplatin, $80 \%$ of aged mice treated with high dose cisplatin survived (Fig 3.3A). In addition, aged mice treated with cisplatin had lost an average of $11.9 \%$ of total body weight at Day 3, but weight loss was not significantly different than cisplatin-treated young mice (Fig 3.1B). These data indicate that aged mice cannot tolerate high dose cisplatin as well as young mice due to mortality, and may allude to worsened AKI in this aged cohort. 
(A)

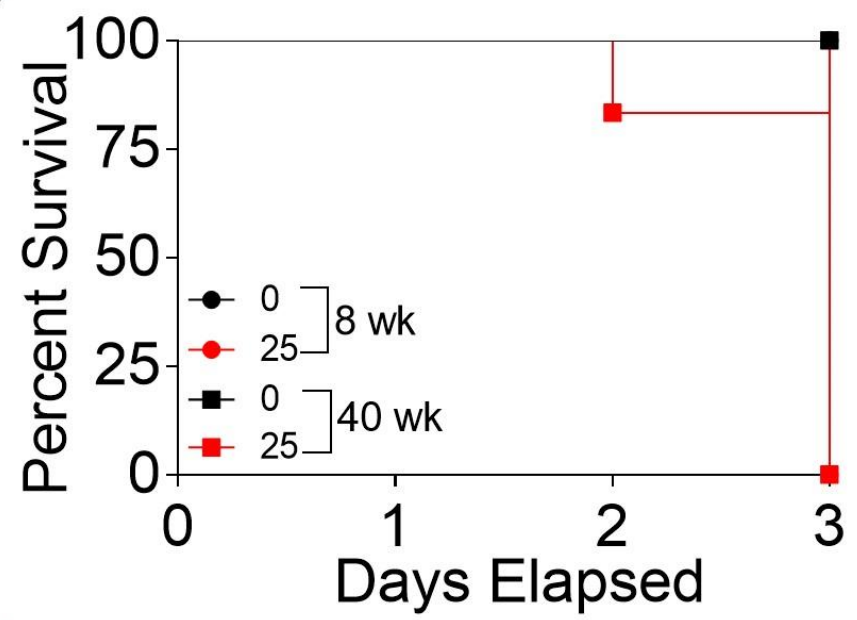

(B)

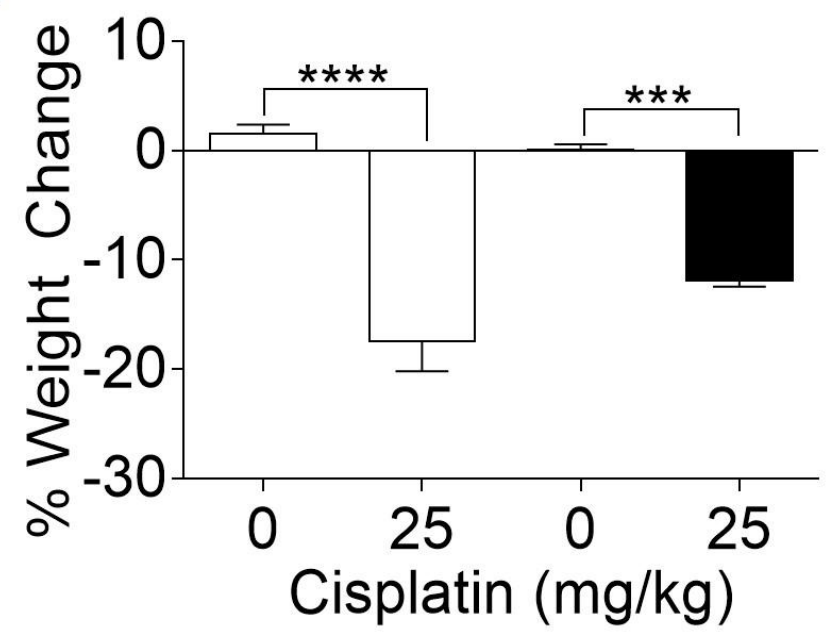

Figure 3.3. Survival and weight loss with the standard dosing regimen of cisplatin. 8 and 40 week old male FVB mice were treated with either $0 \mathrm{mg} / \mathrm{kg}$ or $25 \mathrm{mg} / \mathrm{kg}$ cisplatin once. (A) Percent survival until Day 3. (B) Percent weight change in mice. Data are expressed as mean \pm SEM; $\mathrm{n}=5-10$. Statistical significance was determined by Two-Way ANOVA followed by Tukey post-test. ${ }^{* * *} \mathrm{P}<0.001, * * * * \mathrm{P}<0.0001$. 


\section{BUN and kidney injury levels increase in 8 and 40 week old mice treated with the}

standard dosing regimen of cisplatin. During AKI, there is a rapid decline in kidney function that leads to the buildup of waste products in the blood which can be measured by either SCr or BUN. Kidney function declines with normal aging, thus we hypothesized that aged mice would have increased baseline BUN levels compared to young mice. We further hypothesized that, when treated with the standard dosing regimen of cisplatin, aged mice would have a more severe loss of kidney function compared to young mice treated with the same dosing regimen. BUN levels significantly increased in both young and aged cisplatin-treated mice, but BUN levels were comparable between these two groups (Fig 3.4A). In addition, BUN was not basally elevated in aged mice compared to young mice (Fig 3.4A).

Without overt changes to BUN levels, kidney injury can be detected by more sensitive biomarkers like NGAL and KIM-1. NGAL levels in urine increased approximately 15000 -fold in young mice treated with cisplatin, but only 14.6 -fold in aged mice compared to their respective control (Fig 3.4B). Furthermore, NGAL levels were elevated in aged mice compared to young mice treated with $0 \mathrm{mg} / \mathrm{kg}$ cisplatin (Fig 3.4B). Kim-1 mRNA levels were elevated in young mice treated with cisplatin, and were further increased in aged mice treated with cisplatin. Taken together, these data indicate that treatment of aged and young mice with the standard dosing regimen of cisplatin leads to a comparable loss of function, but higher levels of NGAL in young mice, and higher levels of Kim- 1 in aged mice. Since NGAL is also a marker of inflammation, aged mice may have an altered inflammatory response to cisplatin treatment. 
(A)

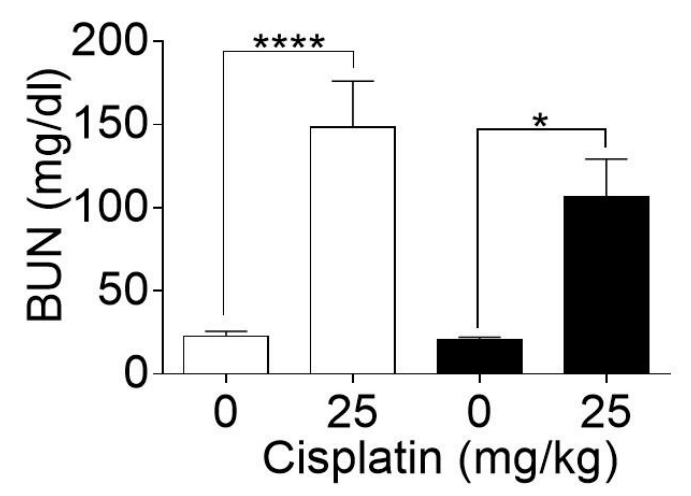

(B)

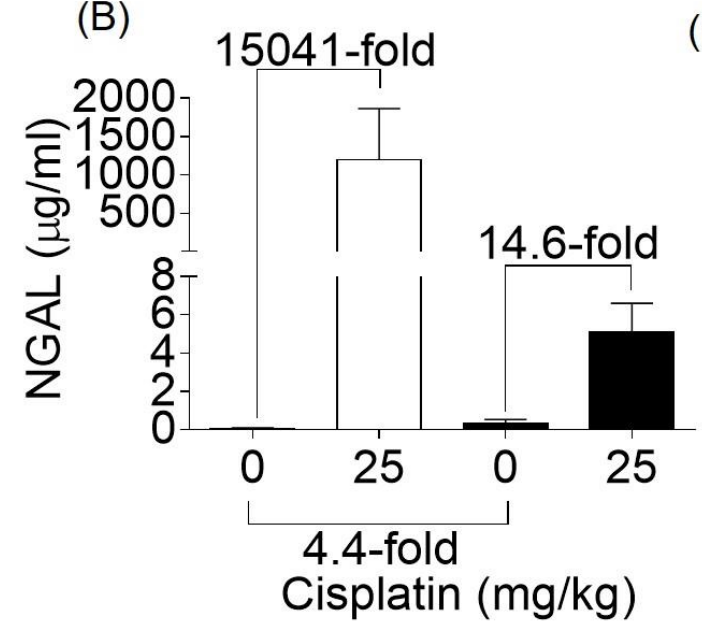

(C)

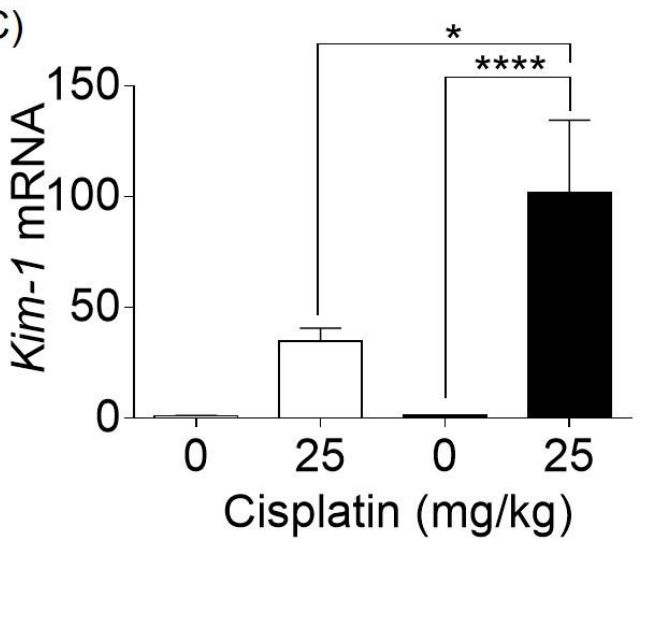

Figure 3.4. Changes in kidney function and injury markers with the standard dosing regimen of cisplatin. 8 and 40 week old male FVB mice were treated with either $0 \mathrm{mg} / \mathrm{kg}$ or $25 \mathrm{mg} / \mathrm{kg}$ cisplatin once. (A) BUN levels measured in plasma. (B) NGAL levels measured in urine. (C) Kim-1 mRNA levels measured in the kidney cortex via QRTPCR. Data are expressed as mean $\pm \mathrm{SEM} ; \mathrm{n}=5-10$. Statistical significance was determined by Two-Way ANOVA followed by Tukey post-test. ${ }^{*} \mathrm{P}<0.05,{ }^{* * *} \mathrm{P}<0.0001$. 


\section{Inflammatory cytokines and chemokines increase in 8 and 40 week old mice treated}

with the standard dosing regimen of cisplatin. The activation of inflammatory cytokines and chemokines plays a major role in the pathogenesis of cisplatin-induced AKI. Of note, TNF $\alpha$ increases early in kidney injury, and initiates the inflammatory response. Tnf $\alpha$ mRNA levels increased approximately 10 -fold in young mice treated with cisplatin, but only 4.7-fold in aged mice (Fig 3.5A). Il6, another pro-inflammatory cytokine, increased 30.8-fold in young mice, but only 11.3-fold in aged mice (Fig 3.5B). Cxcll and Mcp-1, chemokines involved in the recruitment of immune cells to the site of injury, both increased in young and aged mice treated with cisplatin (Fig 3.5C,D). Interestingly, $M c p-1$ levels were basally elevated in aged mice compared to young mice (Fig 3.5D). Thus, these data indicate that aged mice have less of an inflammatory cytokine response with high dose cisplatin without changes in chemokine levels. 


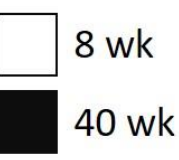

(A)

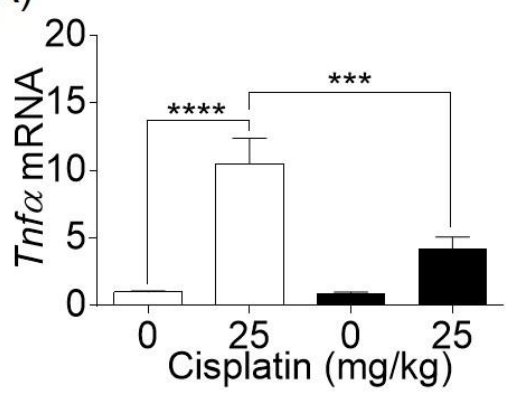

(C) 80

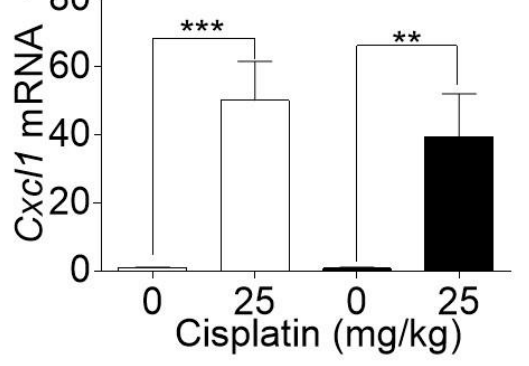

(B)

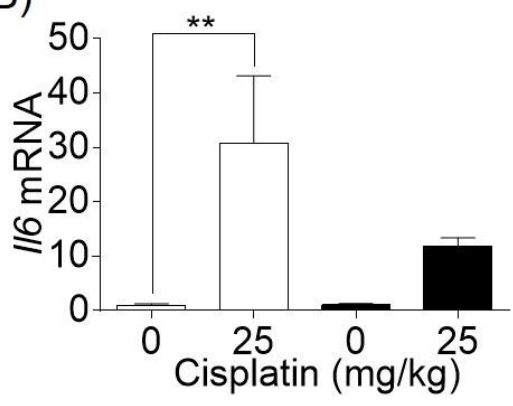

(D)

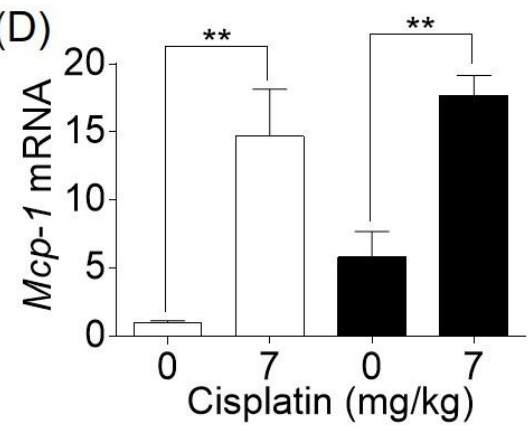

Figure 3.5. Changes in inflammatory cytokine and chemokine levels with standard dosing regimen of cisplatin. 8 and 40 week old male FVB mice were treated with either 0 or $25 \mathrm{mg} / \mathrm{kg}$ cisplatin once. Levels of (A) Tnfa, (B) Il6, (C) Cxcll, and (D) $M c p-1$ were measured in the kidney cortex via real-time QRTPCR. Data are expressed as mean $\pm \mathrm{SEM} ; \mathrm{n}=5-10$. Statistical significance was determined by Two-Way ANOVA followed by Tukey post-test. ${ }^{*} \mathrm{P}<0.05,{ }^{*} \mathrm{P}<0.01$, $* * * \mathrm{P}<0.001$, and $* * * * \mathrm{P}<0.0001$ 


\section{Endoplasmic reticulum (ER) stress and apoptosis occur in the kidney in 8 and 40}

week old mice treated with the standard dosing regimen of cisplatin. A hallmark of AKI is apoptotic cell death mainly affecting tubular epithelial cells. Furthermore, in I/R models of AKI, ER stress occurs thereby activating intrinsic cell death pathways. CHOP is a transcription factor that is elevated during ER stress. Aged mice had high, basal protein levels of CHOP compared to young mice (Fig 3.6). When treated with cisplatin, CHOP levels increased in both young and aged mice (Fig 3.6). Cleaved caspase 3 is an established marker of apoptosis. Treatment with cisplatin led to an increase in CC3 levels in both aged and young mice (Fig 3.6). Taken together, these data indicate that while aged mice have high basal levels of ER stress, the levels of apoptosis that occur with cisplatin treatment in young and aged mice are comparable, indicating aged mice do not have exacerbated AKI. 


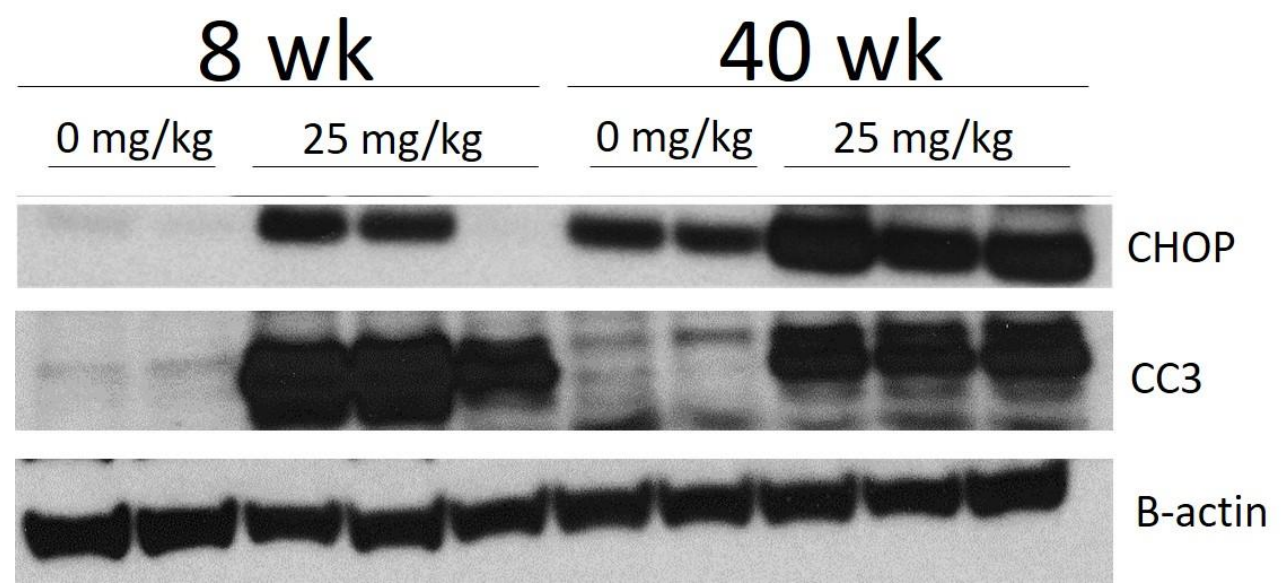

Figure 3.6. Endoplasmic reticulum stress and apoptosis with standard dosing regimen of cisplatin. 8 and 40 week old male FVB mice were treated with either 0 $\mathrm{mg} / \mathrm{kg}$ or $25 \mathrm{mg} / \mathrm{kg}$ cisplatin once. Protein levels of $\mathrm{CHOP}, \mathrm{CC} 3$, and $\beta$-actin were determined in kidney cortex homogenate via Western blot. 
Overall survival and weight loss in 8 and 40 week old mice treated with the repeated dosing regimen of cisplatin. We have previously shown that 8 week old mice treated with repeated, low dose cisplatin ( $7 \mathrm{mg} / \mathrm{kg}$ once a week for four weeks) had a $100 \%$ survival rate at Day 24, and that these mice can be aged out at least six months after treatment with minimal weight loss. Both young and aged mice treated with either repeated dosing of 0 or $7 \mathrm{mg} / \mathrm{kg}$ cisplatin had a 100\% survival rate at Day 24 (Fig 3.7A). In addition, total weight loss for cisplatin treated mice in both age groups was less than $10 \%$ (Fig 3.7B). These data indicate that there is no difference in survival or weight loss in young and aged mice treated with the repeated dosing regimen of cisplatin. 
(A)

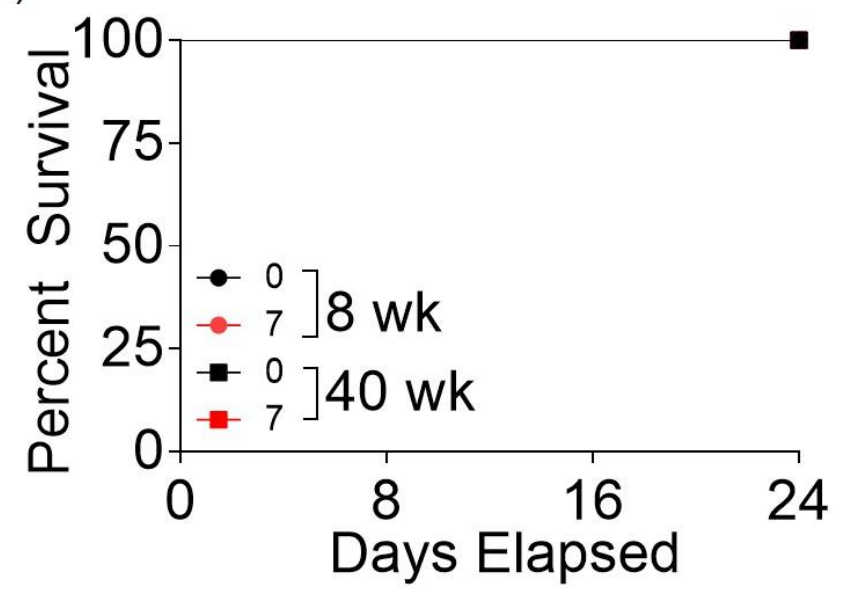

(B)

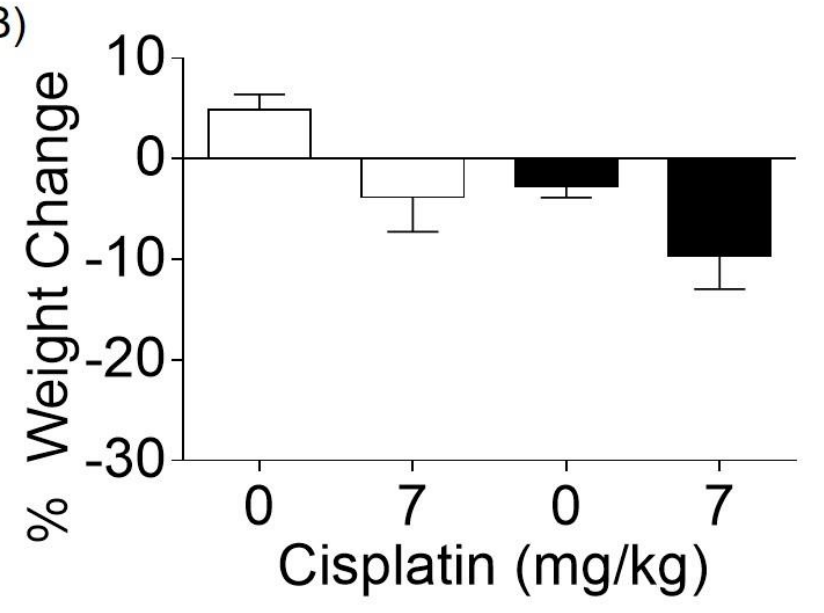

Figure 3.7. Survival and weight loss with the repeated dosing regimen of

cisplatin. 8 and 40 week old male FVB mice were treated with either $0 \mathrm{mg} / \mathrm{kg}$ or 7 $\mathrm{mg} / \mathrm{kg}$ cisplatin once a week for four weeks. (A) Percent survival until Day 24. (B) Percent weight change in mice. Data are expressed as mean \pm SEM; $n=5-10$.

Statistical significance was determined by Two-Way ANOVA followed by Tukey post-test. 


\section{BUN and NGAL levels increase in 8 and 40 week old mice treated with the repeated}

dosing regimen of cisplatin. We have previously shown that repeated, low dose cisplatin treatment causes a slight decline in kidney function, and an increase in NGAL levels indicative of kidney injury. BUN levels in young and aged mice increased with cisplatin treatment, although this increase was not significant (Fig 3.8A). NGAL levels increased 51.2-fold in young mice, and only 9.3-fold in aged mice treated with cisplatin. Basally, NGAL levels were elevated 6.8-fold in aged mice compared to young mice (Fig 3.8B). KIM-1 is also a sensitive marker of kidney injury. Kim-1 mRNA levels were increased 130 -fold in young mice and 72 -fold in aged mice compared to respective controls (Fig 3.8C). Thus, data suggests that while injury markers are already elevated basally in aged mice, there is no difference in the level of kidney injury or loss of function caused by repeated cisplatin treatment of young and aged mice. 
(A)
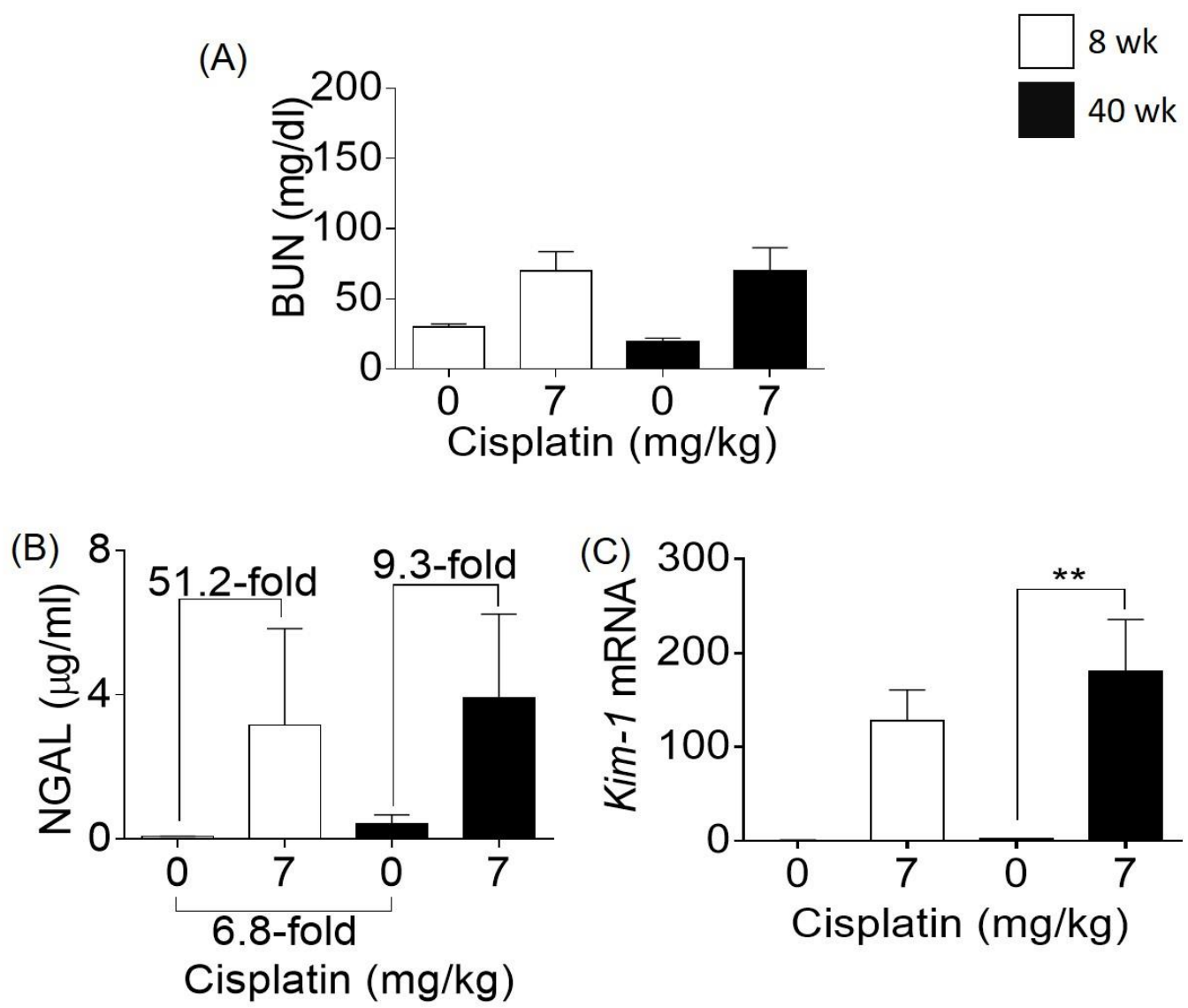

Figure 3.8. Changes in kidney function and injury markers with the repeated dosing regimen of cisplatin. 8 and 40 week old male FVB mice were treated with either $0 \mathrm{mg} / \mathrm{kg}$ or $7 \mathrm{mg} / \mathrm{kg}$ cisplatin once a week for four weeks. (A) BUN levels measured in plasma. (B) NGAL levels measured in urine. (C) Kim-1 levels measured in kidney cortex via real-time QRTPCR. Data are expressed as mean $\pm \mathrm{SEM}$; $\mathrm{n}=5$ 10. Statistical significance was determined by Two-Way ANOVA followed by Tukey post-test. ${ }^{* *} \mathrm{P}<0.01$. 


\section{Inflammatory cytokines and chemokines increase in 8 and 40 week old mice treated}

with the repeated dosing regimen of cisplatin. Chronic inflammation is a major driver of fibrosis. We have shown that many of the same inflammatory cytokines and chemokines that play a role in cisplatin-induced AKI are also involved in the progression of kidney injury to CKD. Tnf $\alpha$ mRNA levels increased 6-fold in young mice treated with cisplatin, but only 4-fold in aged mice (Fig 3.9A). Il6 levels were elevated 8.8-fold in young mice treated with cisplatin, but only 1.8-fold in aged mice (Fig 3.9B).

Inflammatory chemokines $C x c l 1$ and $M c p-1$ significantly increased in both young and aged mice treated with cisplatin, and $M c p-1$ was basally elevated in aged mice compared to young mice (Fig 3.9C,D). Thus, there is less of an inflammatory cytokine response in aged mice treated with cisplatin, similar to the trend seen with the standard dosing model (Fig 3.6). 
$\square$ wk

$40 \mathrm{wk}$

(A)

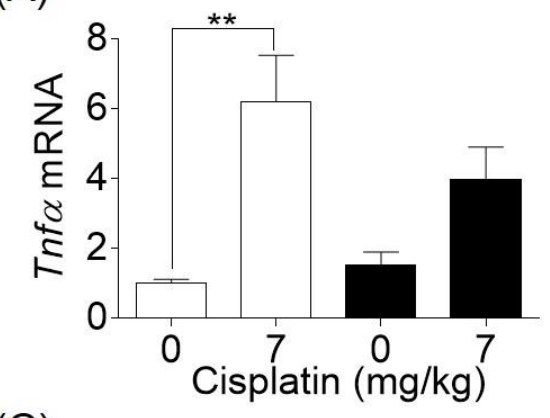

(C)

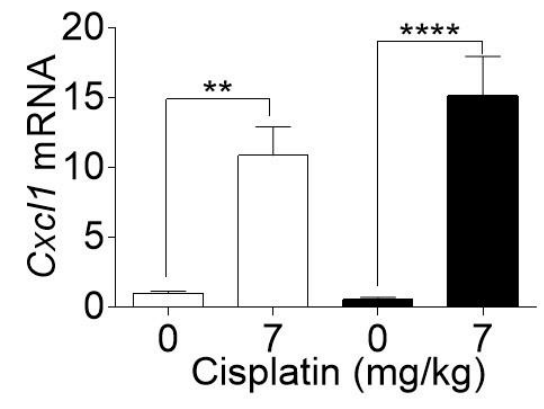

(B)

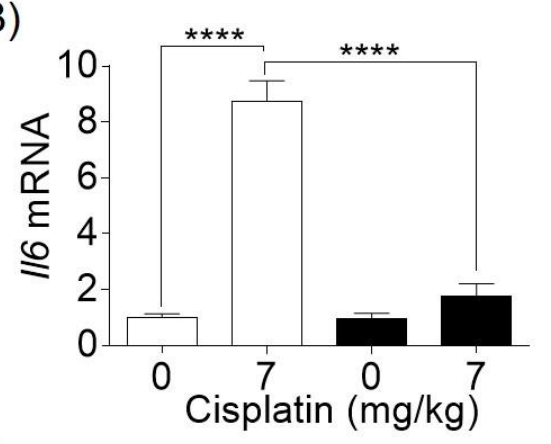

(D)

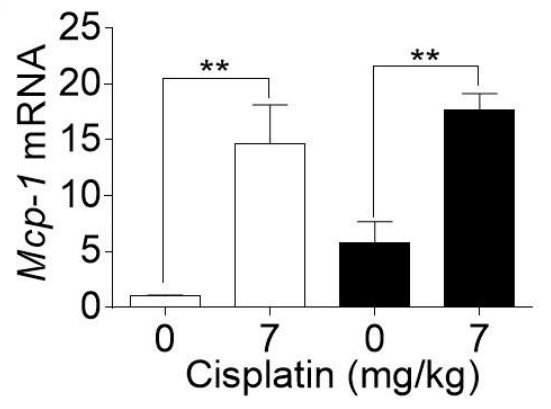

Figure 3.9. Changes in inflammatory cytokine and chemokine levels with the repeated dosing regimen of cisplatin. 8 and 40 week old male FVB mice were treated with either $0 \mathrm{mg} / \mathrm{kg}$ or $7 \mathrm{mg} / \mathrm{kg}$ cisplatin once a week for four weeks. Levels of (A) Tnfa, (B) Il6, (C) Cxcll, and (D) $M c p-1$ were measured in the kidney cortex via real-time QRTPCR. Data are expressed as mean \pm SEM; $n=5-10$. Statistical significance was determined by Two-Way ANOVA followed by Tukey post-test. $* * \mathrm{P}<0.01$ and $* * * * \mathrm{P}<0.0001$ 


\section{Fibrosis develops in 8 and 40 week old mice treated with the repeated dosing}

regimen of cisplatin. Our group and others have demonstrated that repeated, low dose administration of cisplatin induces fibrosis. Pathways of fibrogenesis are basally activated with normal kidney aging, and may contribute to the increased rate of CKD seen in aged populations. Thus, we hypothesized that aged mice treated with the repeated dosing regimen of cisplatin would have an exacerbated fibrotic phenotype compared to young mice treated with cisplatin. Activation of the TGF $\beta$ signaling pathway is one of the major contributors to the development of fibrosis. Furthermore, fibrosis is marked by an increase in ECM components, namely fibronectin and collagen. Both TGF $\beta$ and fibronectin levels increased in young and aged mice treated with cisplatin (Fig 3.10A). Total collagen deposition as evidenced by SR/FG indicated that aged mice had higher collagen levels basally compared to young mice (3.8-fold increase) (Fig 3.10B). However, treatment with cisplatin led to a 5.3-fold increase in collagen in young mice, but only a 1.5-fold increase in aged mice, although overall collagen deposition levels were comparable between the two groups (approximately 11\% Sirius red positive pixels) (Fig 3.10B).

Myofibroblasts produce collagen during fibrogenesis. Immunohistochemistry staining for $\alpha \mathrm{SMA}$ indicated more myofibroblasts were present basally in aged mice compared to young mice (4.1-fold increase) (Fig 3.10C). $\alpha \mathrm{SMA}+$ myofibroblasts increased 4.2-fold in young mice treated with cisplatin, but only 1.6-fold in aged mice (Fig 3.10C). Overall, fibrosis was not worsened in aged mice treated with repeated dosing of cisplatin, even though $\alpha \mathrm{SMA}+$ myofibroblasts and collagen deposition were basally elevated. 
(A)

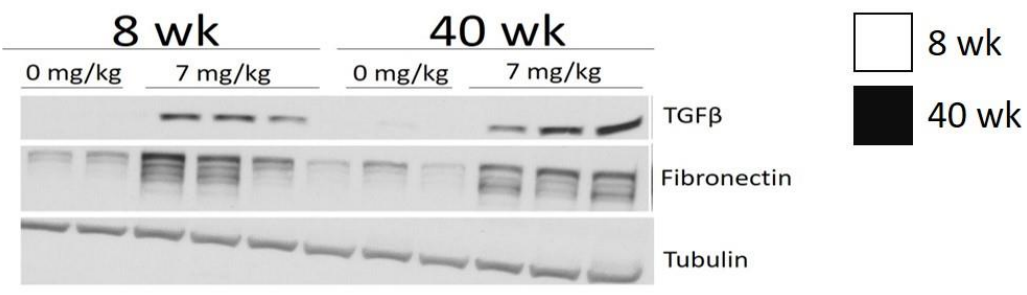

(B)
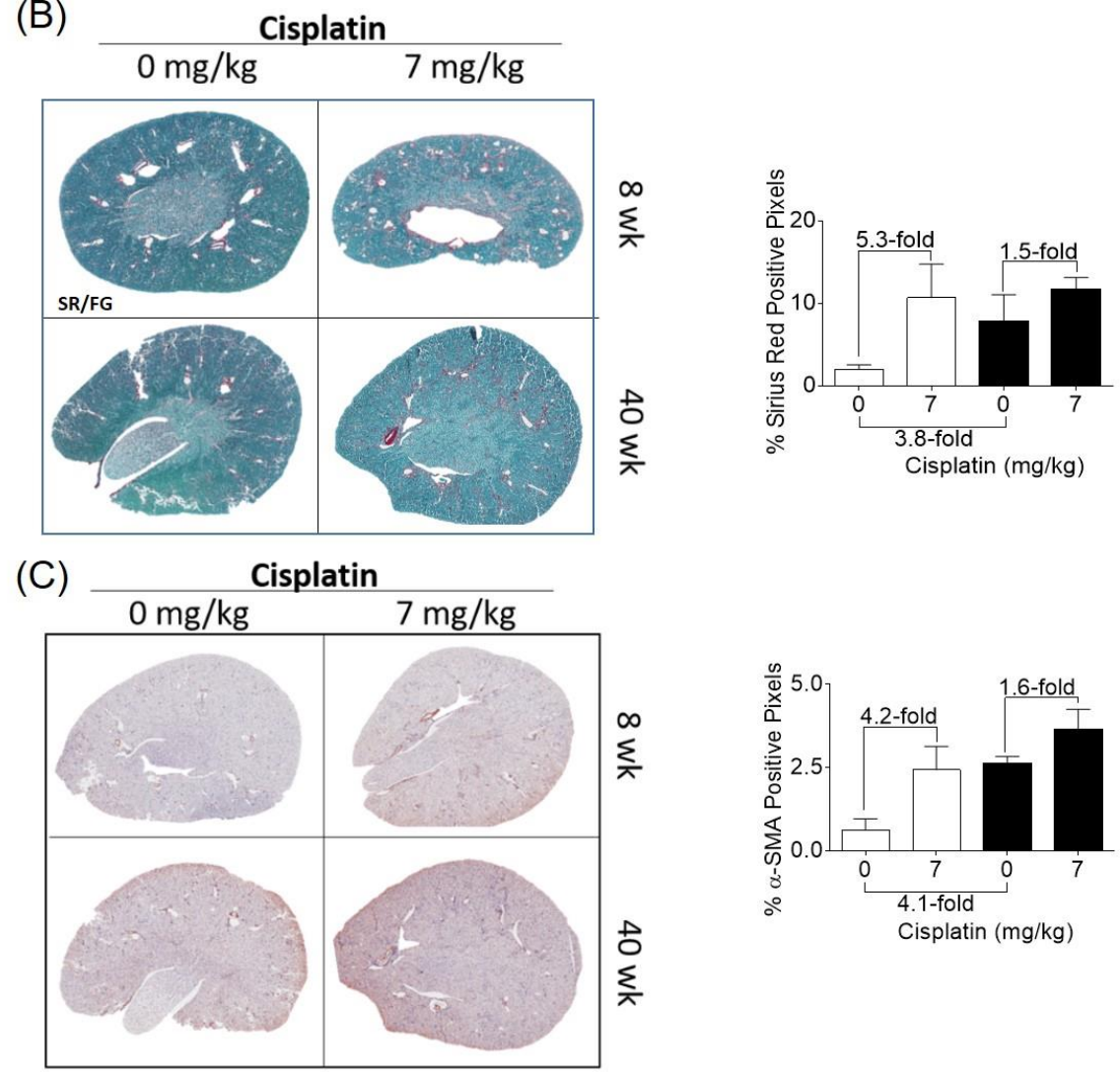
Figure 3.10. Fibrosis develops with the repeated dosing regimen of cisplatin. 8 and 40 week old male FVB mice were treated with either $0 \mathrm{mg} / \mathrm{kg}$ or $7 \mathrm{mg} / \mathrm{kg}$ cisplatin once a week for four weeks. (A) Protein levels of TGF $\beta$ and fibronectin were determined in kidney cortex homogenate with Western blot. (B) Total collagen levels were determine via SR/FG staining and quantified as \% Sirius red positive pixels. (C) Presence of myofibroblasts was determined by aSMA IHC, and quantified as \% aSMA positive pixels. Data are expressed as mean \pm SEM; $n=5-10$. Statistical significance was determined by Two-Way ANOVA followed by Tukey post-test. 


\section{Increased CD3 + $T$ cells and F4/80 + macrophages in the kidneys of aged mice treated}

with the repeated dosing regimen of cisplatin. Immune cells after kidney injury play a dual role in exacerbating injury, as well as initiating adaptive repair responses. We have previously shown that with repeated dosing of cisplatin, there is an increase in macrophages in the kidney that is associated with worsening fibrosis. Aged mice treated with cisplatin had increased F4/80+ staining for macrophages compared to cisplatintreated young mice (Fig 3.11A). ARG-1 and iNOS are markers for M2 and M1 macrophages, respectively. M1 macrophages are pro-inflammatory and lead to worsened injury, but M2 macrophages are involved in adaptive repair due to their antiinflammatory phenotype. Inos mRNA levels did not increase in either cohort of mice treated with cisplatin; however, Arg-1 mRNA levels increased 5.3-fold in young mice, but only 1.8-fold in aged mice (Fig 3.11B,C).

T cells (CD3+) are known to contribute to both cisplatin-induced AKI, and the development of CKD. CD3 $+\mathrm{T}$ cells also increased in aged mice treated with cisplatin compared to young mice (Fig 3.11D). Furthermore, these T cells formed clusters in aged mice, but not in young mice. Taken together, IHC indicates there is more immune cell infiltration in old mice treated with cisplatin, even though fibrosis is not worsened in these mice (Fig 3.9C). 


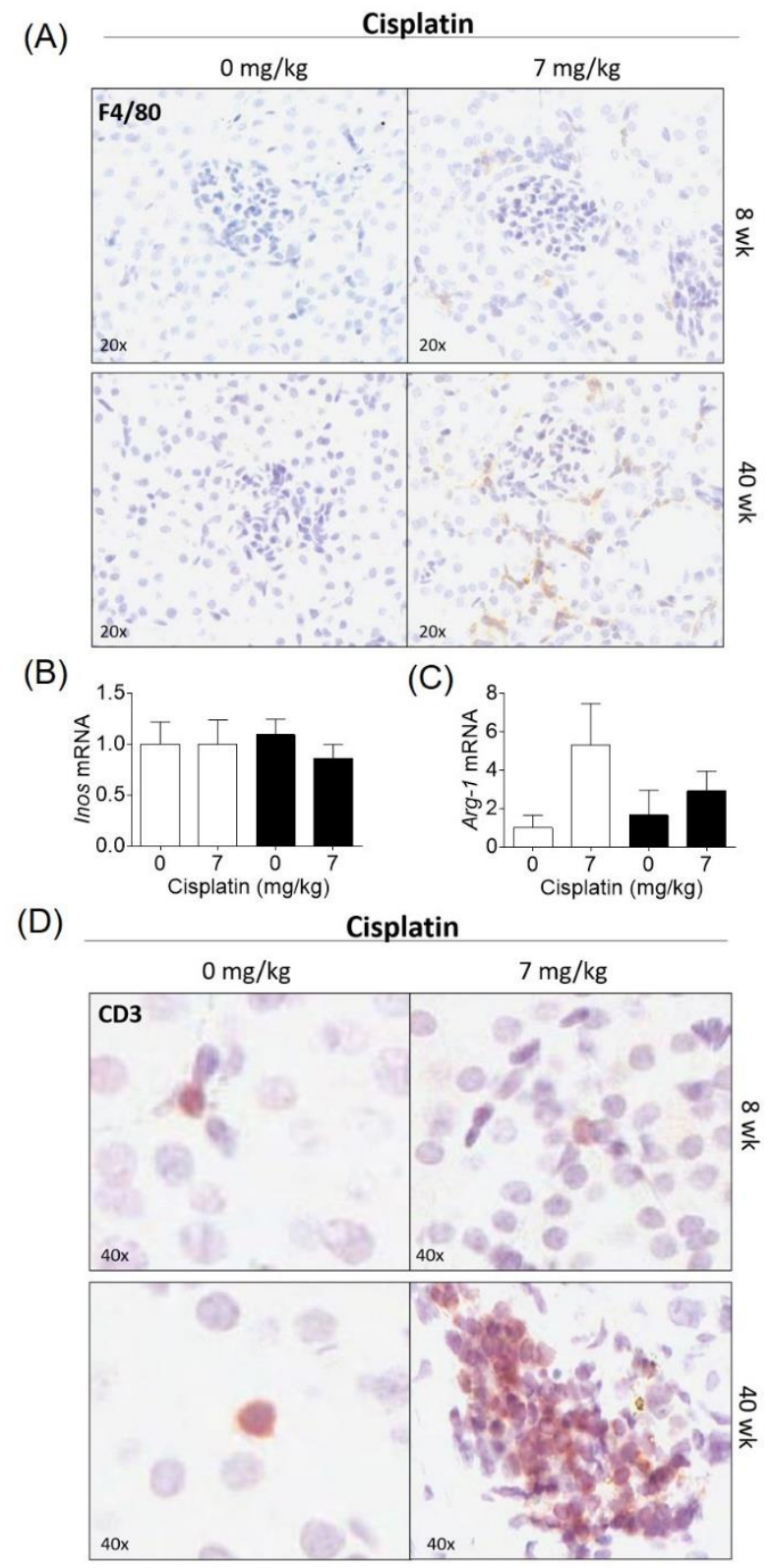

Figure 3.11. Immune cells in the kidney with the repeated dosing regimen of cisplatin. 8 and 40 week old male FVB mice were treated with either $0 \mathrm{mg} / \mathrm{kg}$ or 7 $\mathrm{mg} / \mathrm{kg}$ cisplatin once a week for four weeks. (A) Total macrophages were determined via F4/80 IHC. Levels of (B) Inos and (C) Arg-1 were determined in the kidney cortex via real-time QRTPCR. (D) Total T cells were determined via CD3 IHC. Data are expressed as mean $\pm \mathrm{SEM} ; \mathrm{n}=5-10$. Statistical significance was determined by Two-Way ANOVA followed by Tukey post-test. 


\section{DISCUSSION}

Renal aging is often marked by the development of glomerulosclerosis, tubular atrophy, increased cellular senescence, the development of interstitial fibrosis, and total nephron loss $(1,95)$. These physiological changes ultimately culminate in decreased GFR, the inability to properly conserve and secrete sodium, and difficulties in proper concentration or dilution of urine $(53,95)$. In the context of cisplatin-induced nephrotoxicity, Wen et al found that lung cancer patients receiving one dose of cisplatin had a $9.2 \%$ decrease in GFR, and that age was the only risk factor examined to have a significant relationship with decline in kidney function after the first round of cisplatin treatment (96). However, these patients had also received other chemotherapeutics known to cause kidney injury. Of note, 83 patients that received cisplatin also received gemcitabine, a well-known inducer of nephrotoxicity $(96,97)$. Studies such as this highlight the need to better understand the true contribution of cisplatin to worsened kidney injury with aging.

Furthermore, most experimental studies that examine the effects of aging in regards to increased injury are performed in very old animals, making it difficult to translate these findings into the clinical setting where the median age of all cancer diagnoses is 65 years old (52). Wen et al demonstrated that 18 month old C57BL/6J mice developed more severe kidney injury when treated with $15 \mathrm{mg} / \mathrm{kg}$ cisplatin compared to 3 month old mice (96). These aged mice had increased BUN levels when treated with cisplatin, an increase in TLR4 mediated signaling, increased cisplatin accumulation in the kidney, and a 35\% decrease in protein levels of MATE1, an efflux transporter that is responsible for removal of cisplatin from the kidney (96). 
Despite concern for increased susceptibility to kidney injury in the aged population, several clinical studies have indicated that aging may not be as great of a concern. First, the rate of nephrotoxicity/AKI in pediatric cancer patients treated with cisplatin is comparable to the rate seen in adults. Skinner et al found that $50 \%$ of children treated with either cisplatin or carboplatin had nephrotoxicity post-treatment, ad $36.4 \%$ of these patients had a decreased GFR indicative of CKD (32). The rate of cisplatin nephrotoxicity in adult cancer patients ranges from $20-30 \%$, and approximately $20 \%$ of all adult patients diagnosed with AKI will progress to $\operatorname{CKD}(9,10)$.

In a study by Thyss et al, patients aged 80-87 without underlying cardiovascular disease did not have an increased rate of cisplatin nephrotoxicity compared to younger populations (aged 55 or less) receiving a similar dosing regimen (98). This study further highlighted that comorbidities such as cardiovascular disease may contribute to kidney function changes that are often associated with normal aging, and may attribute to increased susceptibility to cisplatin nephrotoxicity.

In addition, Hrushesky et al reported that patients 60-70 years of age treated with cisplatin had an average $21 \mathrm{ml} / \mathrm{min}$ decline in creatinine clearance, but this decline was $30 \mathrm{ml} / \mathrm{min}$ in patients aged 50 or younger, indicating a greater loss of kidney function in the younger cohort of patients (99). Hrushesky et al concluded that these 60-70 year olds may be slightly protected from cisplatin nephrotoxicity because the external medulla is highly preserved despite aging, and that this was the main site of cisplatin concentration in older patients (99). Finally, with aging there was no change in overall clearance of cisplatin, suggesting concern for treating patients of advanced age may not be as serious as previously believed. 
In this study, we utilized 40 week old FVB mice as our aged cohort. This represents a middle age cohort of mice, as the average lifespan of FVB mice is 16-20 months. We believe using this age of mouse better reflects the cohort of patients who are diagnosed with cancer that are eligible to receive cisplatin treatment. Our data in aged mice treated with cisplatin indicate that there is a difference in both the inflammatory response and immune cell recruitment after injury, without worsening of kidney injury or an overt change in loss of kidney function.

Of note, we saw no age-dependent changes in basal BUN levels, nor difference in BUN between 8 and 40 week old mice treated with either dosing regimen of cisplatin. This challenges the belief that normal kidney aging leads to a decline in kidney function, and highlights that changes in kidney function in older patients may be due to underlying comorbidities. Furthermore, although nephron mass is lost with normal aging, compensatory hypertrophy of the remaining nephrons may help maintain normal kidney function, especially when kidney injury occurs (99).

When measuring kidney injury with sensitive biomarkers, we found that aged mice treated with the standard dosing regimen of cisplatin had much lower levels of NGAL overall, and these levels were comparable to those in 8 and 40 week old mice treated with the repeated dosing regimen of cisplatin. However, basal levels of NGAL were higher in aged mice compared to young mice. Recent studies have indicated that NGAL may be a better biomarker for inflammation, especially as TNF $\alpha$ can directly upregulate NGAL expression (100). Thus, NGAL may be indicative of an altered inflammatory response with cisplatin in aged mice. Indeed, Tnfa mRNA levels in 40 week old mice treated with the standard dosing regimen of cisplatin were significantly 
less than those of 8 week old mice treated with the same regimen. This coincides with data presented in an I/R study by Jang et al, in which TNFa levels were lower with ischemic injury in both 6 and 12 month old C57BL/6J mice compared to 3 month old mice with the same injury (101). This trend was also apparent with the repeated dosing regimen of cisplatin. Furthermore, $I l 6$ mRNA levels were lower in 40 week old mice compared to 8 week old mice treated with either dosing regimen of cisplatin. However, in a study of normal aging and changes to the immune micromilieu, IL6 levels were basally increased in both 6 and 12 month old mice, although these elevated levels did not result in worsened ischemic injury in aged mice (101). Taken together, our data indicate that aged mice have a decreased inflammatory response to cisplatin treatment.

Chronic inflammation also contributes to renal fibrosis (1). Forty week old mice had increased collagen deposition and more myofibroblasts present compared to 8 week old mice basally. However, treatment with repeated dosing of cisplatin did not lead to overt collagen deposition, or the presence of more myofibroblasts in aged mice. This outcome may correlate with the lack of a robust inflammatory response with cisplatin treatment. Interestingly, there was more immune cell infiltration of CD3+ T cell and F4/80+ macrophages in the kidneys of 40 week old mice treated with the repeated dosing regimen of cisplatin than in 8 week old mice. Classically, a robust inflammatory response corresponds to increased immune cell infiltration which can further drive the inflammatory response. However, both $\mathrm{T}$ cells and macrophages play diverse roles in kidney injury, and it has been reported that $\mathrm{T}$ lymphocytes have decreased function with aging, adapting an "immuosenescent" phenotype $(1,9)$. Of note, we saw an increase in M2 macrophages as measured by Arg-1 mRNA expression. Arg-1 levels were higher in 
young mice treated with repeated doses of cisplatin, even though total macrophages were higher in the aged group treated with cisplatin. This finding may further support an immunosuppressive phenotype, as we hypothesize that macrophages in aged mice have less activity and thus lower production of ARG-1, although this must be confirmed in future studies.

Taken together, the cisplatin dosing regimens discussed here represent both AKI and $\mathrm{CKD}$, two disease states that are thought to be more likely to occur in aged populations. Overall, this study indicates that aging does not greatly alter kidney injury or changes in function with both the standard and repeated dosing regimens of cisplatin. Thus, treating older patients with cisplatin may not result in a more severe kidney injury phenotype or increased rate of nephrotoxicity. Furthermore, worsened injury and higher susceptibility to injury may be the result of comorbidities aside from age, such as underlying cardiovascular disease, diabetes, polypharmacy, or genetic polymorphismsfactors that are usually not accounted for in patient aging studies $(1,96)$. 


\section{CHAPTER 4}

\section{TREATMENT WITH REPEATED DOSING OF CISPLATIN CAUSES WORSENED FIBROSIS IN A MOUSE MODEL OF KRAS4BG12D LUNG ADENOCARCINOMA THAT CANNOT BE PREVENTED WITH ERLOTINIB}

\section{INTRODUCTION}

The overall survival of cancer patients has improved over the last ten years, but with this increase in survival comes a potentially greater risk for patients to have longlasting side effects from the cancer itself, or the treatment of cancer. Of note, cancer patients have a higher rate of AKI than critically ill patients without cancer (102). Furthermore, cancer patients often have more severe forms of AKI that will often require renal replacement therapy, and the 28-day mortality rate in these patients ranges from 66$88 \%(102,103)$. In a Danish study of 1.2 million cancer patients, rates of AKI were found to be highest in patients with renal cell carcinoma, leukemia, and liver cancer (103). Of the patients that had one of these types of cancer, the overall one year risk of AKI was $17.5 \%$, and this increased to $27 \%$ over a five year period (103). In addition, $5 \%$ of patients with cancer required dialysis within the first year of diagnosis.

The increased susceptibility to AKI in cancer patients may be attributed to the greater probability of being exposed to known causes of AKI. For example, sepsisinduced AKI is more prevalent in cancer patients (102). Those who develop sepsis are often treated with anti-infectives, of which many cause nephrotoxicity. In addition, 
contrast-induced nephrotoxicity is also high in cancer patients because they often undergo imaging procedures during the initial cancer screening and course of treatment.

Cancer patients also have a higher pre-test probability for certain causes of AKI. For one, tumor lysis syndrome (TLS)-induced AKI is specific to cancer patients (102). Tumor lysis syndrome occurs most often during the treatment of cancer, as dying cancer cells release their intracellular components into the bloodstream (104). This leads to the development of hyperkalemia, hypocalcemia, hyperphosphatemia, and hyperuricemia. Furthermore, the formation of crystals of uric acid and calcium phosphate can cause intratubular obstructions when filtered through the kidney (104). This in turn can lead to a decline in kidney function and cause an inflammatory response. The combination of crystal obstruction and a robust inflammatory response is associated with renal vasoconstriction and reduced renal blood flow, ultimately culminating in severe kidney injury and functional loss.

The use of nephrotoxic chemotherapeutics and other oncotherapies is also specific to cancer patients. Of note, cisplatin is one of the most widely used chemotherapeutics for the treatment of solid tumor cancers. Approximately one third of cancer patients will develop AKI after the first dose of cisplatin, and 20-30\% will develop nephrotoxicity throughout the course of treatment with cisplatin, regardless of the type of cancer $(9,10)$. Since only cancer patients receive cisplatin treatment, it is important to study cisplatininduced AKI experimentally in a mouse model of cancer. However, work in this field is very limited.

In a seminal study by Pabla et al, 7 and 8 week old athymic female mice were injected with ovarian cancer cells subcutaneously. After tumors formed, mice were 
treated with $10 \mathrm{mg} / \mathrm{kg}$ cisplatin weekly (105). Only $30 \%$ of mice with ovarian cancer survived to week 4 with cisplatin treatment, and none were able to receive a fifth dose of cisplatin. These mice also had severe loss of renal function as measured by $\mathrm{SCr}$ and BUN, and a high level of apoptosis in the kidney (105). Utilizing a subcutaneous model of lung adenocarcinoma, Ravichandran et al reported that mice treated with $10 \mathrm{mg} / \mathrm{kg}$ cisplatin once a week for four weeks developed severe kidney injury as measured by NGAL levels in the serum, decreased kidney function, and high levels of acute tubular necrosis and apoptosis (55).

Both studies described here utilize xenograft or subcutaneous models of cancer, which have several limitations for studying the systemic effects of cancer. To address these limitations for incorporating cancer into a clinically relevant mouse model of cisplatin-induced kidney injury, a better approach would be to use a transgenic mouse model with a driver cancer mutation relevant to human cancers. The use of a transgenic model would provide a way to examine cancer in its original location in a mouse with an intact immune system. In addition, the development of cancer in most transgenic mouse models is gradual and better mimics the progression of the human disease. Thus, transgenic mouse models of cancer provide a clinically relevant way to study cancer as a systemic disease, and the potential impact this may have on kidney outcomes with cisplatin-induced kidney injury.

To incorporate the differential effects cancer may have on cisplatin-induced kidney injury, we utilized a Tet-O-Kras4bG12D transgenic mouse model of lung adenocarcinoma. Lung cancer is often treated with low dose cisplatin, making it a relevant cancer to study. Furthermore, while some lung cancers are associated with a 
higher risk of TLS-associated AKI, the risk of cisplatin-induced AKI in patient with lung cancer is not well established $(106,107)$. Based on studies that indicate cancer patients have an increased risk of AKI, we hypothesized that mice with lung adenocarcinoma treated with our repeated dosing regimen of cisplatin would have a higher level of kidney injury and subsequent loss of function compared to non cancer mice. As we have established that repeated administration of cisplatin causes fibrosis, and many of the same processes involved in AKI are also involved in the development of fibrosis, we further hypothesized that mice with lung adenocarcinoma treated with cisplatin may have worsened fibrosis as well, compared to mice treated with cisplatin without lung adenocarcinoma.

To test these hypotheses, 40 week old male and female mice with and without lung adenocarcinoma (non cancer and cancer) were treated with either 0 or $7 \mathrm{mg} / \mathrm{kg}$ cisplatin once a week for four weeks, and euthanized on Day 24. Mice with cancer had a lower rate of survival to Day 24, high levels of NGAL in the urine after Dose 1 and 2, and worsened fibrosis compared to non cancer mice treated with the same cisplatin dosing regimen. To address why mice with cancer treated with cisplatin may have worsened fibrosis, we examined the EGFR signaling pathway. Repeated or sustained activation of EGFR is known to induce renal fibrosis in several experimental mouse models $(74,108,109)$. Western blot analysis indicated that cancer mice treated with cisplatin had increased EGFR levels in the kidney, and subsequently high activation of EGFR. Downstream EGFR signaling pathways were also differentially activated in cancer mice treated with cisplatin. Thus, we hypothesized that inhibition of EGFR signaling could prevent the development of fibrosis, and may improve survival outcomes 
in cancer mice. Using erlotinib, an FDA-approved EGFR inhibitor, we found that inhibition of EGFR caused severe AKI in both cancer and non cancer mice treated with cisplatin. Thus, these data indicate that mice with cancer have worsened kidney injury when treated with our clinically relevant dosing regimen of cisplatin that cannot be prevented by pharmacological inhibition of EGFR.

\section{MATERIALS AND METHODS}

Preparation of methylcellulose and erlotinib. For methylcellulose, $100 \mu 1$ Tween 80

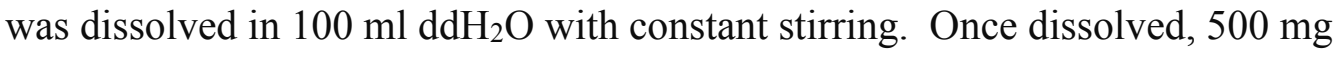
methylcellulose (M0512-100G, Sigma) was added to the solution, and was allowed to stir at room temperature overnight. Methylcellulose solution was then filter sterilized and kept at $4{ }^{\circ} \mathrm{C}$ for 3 months. Erlotinib $\mathrm{HCl}$ (S1023, Selleck Chemicals) was dissolved in methylcellulose at a $5 \mathrm{mg} / \mathrm{ml}$ concentration. Erlotinib solution was sonicated in a $37^{\circ} \mathrm{C}$ sonication bath for 10 minutes, and was then stored at $4^{\circ} \mathrm{C}$ away from light for up to a week.

Animals. For cancer studies, an inducible transgenic mouse model of mutant Kras (Kras4bG12D) lung adenocarcinoma on the FVB/n mouse background was used (Fig 4.1). Briefly, 40 week old male and female single transgenic mice (tet-o-Kras4bG12D or CCSP-rtTA) or double transgenic mice (tet-o-Kras4bG12D-CCSP-rtTA) were intraperitoneally injected at 8:00 a.m. with either saline vehicle $\left(0.9 \%\right.$ saline in $\left.\mathrm{ddH}_{2} \mathrm{O}\right)$ or cisplatin $(7 \mathrm{mg} / \mathrm{kg})$ once a week for four weeks and euthanized at Day 24. Mice were monitored for weight loss or high levels of discomfort/stress on a daily basis, and were euthanized if weight loss was greater than $20 \%$. 
For erlotinib studies, 40 week old male and female single or double transgenic mice were intraperitoneally injected at 8:00 a.m. with either saline vehicle $(0.9 \%$ saline in $\left.\mathrm{ddH}_{2} \mathrm{O}\right)$ or cisplatin $(7 \mathrm{mg} / \mathrm{kg})$ once on Day 1 . These mice also received methylcellulose vehicle or $25 \mathrm{mg} / \mathrm{kg}$ erlotinib intraperitoneally on Day 1-6, and were euthanized on Day 7 (Figure 4.2). Mice were monitored for weight loss or high levels of discomfort/stress on a daily basis, and were euthanized if weight loss was greater than $20 \%$.
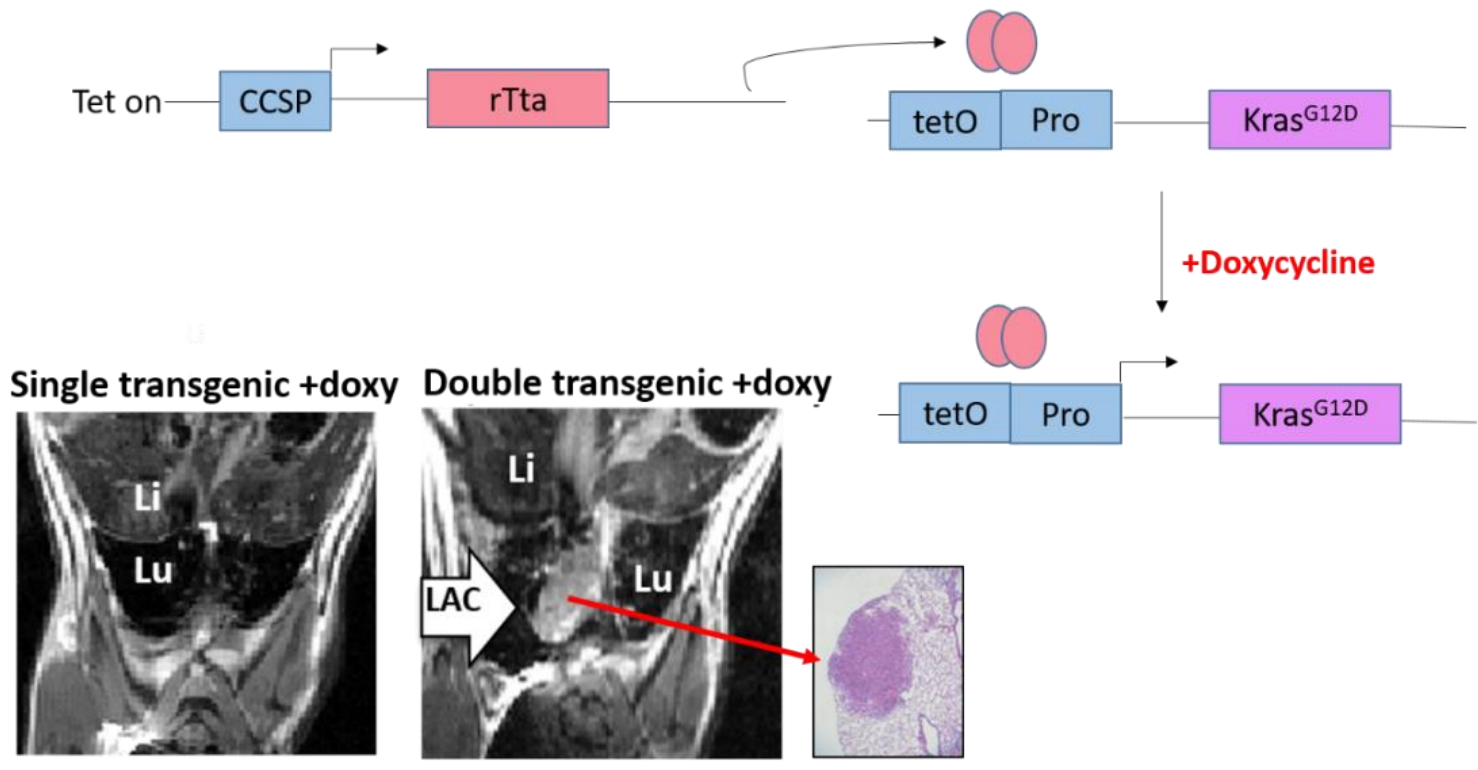

Figure 4.1. Inducible, transgenic model of mutant Kras lung adenocarcinoma.

In this double transgenic model, the reverse tetracycline transactivator (rtTA) is constitutively expressed in type II lung epithelial cells via a Clara cell specific promoter (CCSP). rtTA, in the presence of doxycycline, binds to the tet operon of Kras4bG12D (KrasG12D), thereby activating expression of mutant Kras. Only mice that have both transgenes for CCSP-rtTA/tet-o-Kras4bG12D and that are on a doxycycline diet will develop lung adenocarcinoma. 

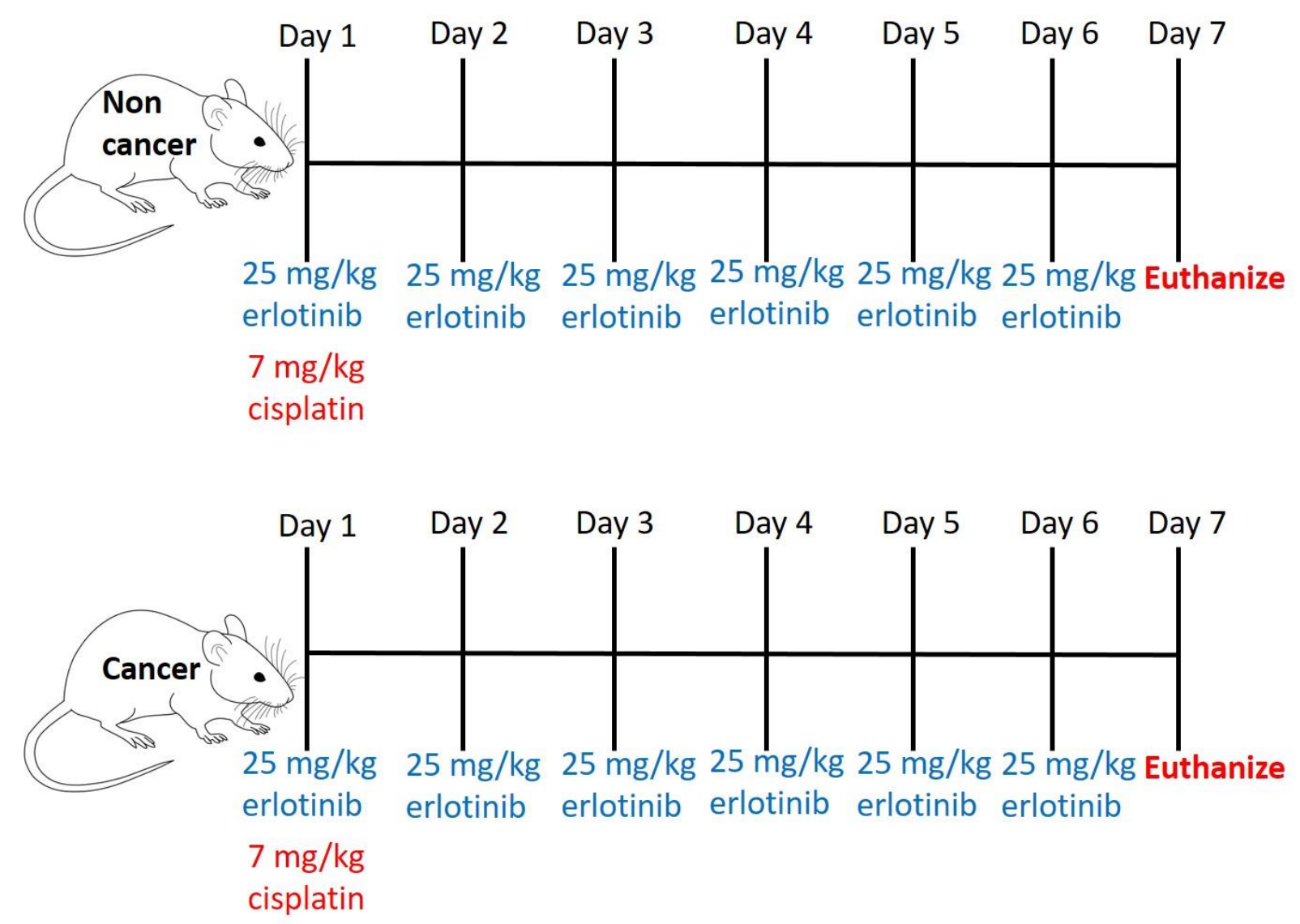

Figure 4.2. Cisplatin and erlotinib dosing regimen. Non cancer and cancer mice were treated with one dose of either 0 or $7 \mathrm{mg} / \mathrm{kg}$ cisplatin on Day 1 . These mice were then treated with either 0 or $25 \mathrm{mg} / \mathrm{kg}$ cisplatin on Days 1-6. On Day 7, mice were euthanized and kidneys, blood, and urine were harvested

Sample collection and storage. Urine was collected and stored at $-80^{\circ} \mathrm{C}$. Blood was collected, and plasma and kidney samples were prepared as previously described in Chapter 2.

BUN determination. Blood urea nitrogen (BUN) levels were determined using a kit from AMS Diagnostics (80146, AMS Diagnostics, Weston, FLA) per the manufacturer's instructions. 
ELISAs. ELISA for neutrophil gelatinase associated lipocalin (NGAL; DY1857, R\&D Systems, Minneapolis, MN) was performed on urine samples using the manufacturers' protocol.

Gene expression. Total RNA was isolated and cDNA was made as outlined in Chapter 2. Gene-specific cDNA was quantified using real-time RTPCR and either pre-designed Taqman assays, or self-designed Sybr assays. The following pre-designed primers were used: (Life Technologies, Grand Island, NY) were used: Tnf $\alpha$ (Mm00443258_m1), Cxcl1 (Mm04207460_m1), Mcp-1 (Mm00441242_m1), and B2m (Mm00437762_m1). Primers were used in combination with iTaq Universal Probes Supermix (172-5134, BioRad, Hercules, CA).

Antibodies. The following antibodies were purchased from Cell Signaling (Beverly, MA) unless otherwise noted: epidermal growth factor receptor (EGFR, no. 9664), pEGFR Y1068 (no. 2895), c-jun N-terminal kinase (JNK, no.9252), pJNK (no.4668), nuclear factor kappa-light-chain enhancer of activated B cells (NFkB, no.8242), pNFkB (no.3033), transforming growth factor (TGF)- $\beta$ (no. 3712S), fibronectin (F3648, SigmaAldrich, St. Louis, MO), and tubulin (SC-23948, Santa Cruz Biotechnology, Dallas, TX).

Protein Isolation/Quantification and Western Blot Analysis. Protein was isolated and quantified as previously published (50). Western blot analysis was performed as previously published, using 1:5000 dilutions for primary antibodies and 1:40000 dilutions for secondary antibodies. Proteins of interest were detected by chemiluminiscence substrate. 
Preparation of kidney sections for histology/IHC. Kidney sections were fixed, embedded, and cut as previously described in Chapter 2.

Immunohistochemistry. aSMA was performed as previously described in Chapter 2 .

Sirius red/fast green staining for total collagen. SR/FG staining was performed as previously described in Chapter 2 .

Statistical analysis. Data are expressed as means \pm SEM for all experiments. Multiple comparisons of normally distributed data sets were analyzed by two way ANOVA, as appropriate, and group means were compared using Tukey posttests. The criteria for statistical differences were: $* \mathrm{P}<0.05,{ }^{*} \mathrm{P}<0.01,{ }^{* * *} \mathrm{P}<0.001$, and $* * * * \mathrm{P}<0.0001$. For erlotinib studies, data sets were analyzed using one way ANOVA.

\section{RESULTS}

\section{Overall survival is decreased in cancer mice treated with repeated dosing of} cisplatin. We have previously published that mice treated with $7 \mathrm{mg} / \mathrm{kg}$ cisplatin once a week for four weeks have a $100 \%$ survival rate to Day 24. Forty week old non cancer mice treated with either 0 or $7 \mathrm{mg} / \mathrm{kg}$ cisplatin had 100\% survival at Day 24 (Fig 4.3). Cancer mice treated with $0 \mathrm{mg} / \mathrm{kg}$ cisplatin had $83 \%$ survival, and cancer mice treated with $7 \mathrm{mg} / \mathrm{kg}$ cisplatin had $47 \%$ overall survival (Fig 4.3 ). These data indicate that cancer mice treated with cisplatin have a substantial decrease in survival rate compared to non cancer, cisplatin treated mice that may be due to worsened kidney injury. 


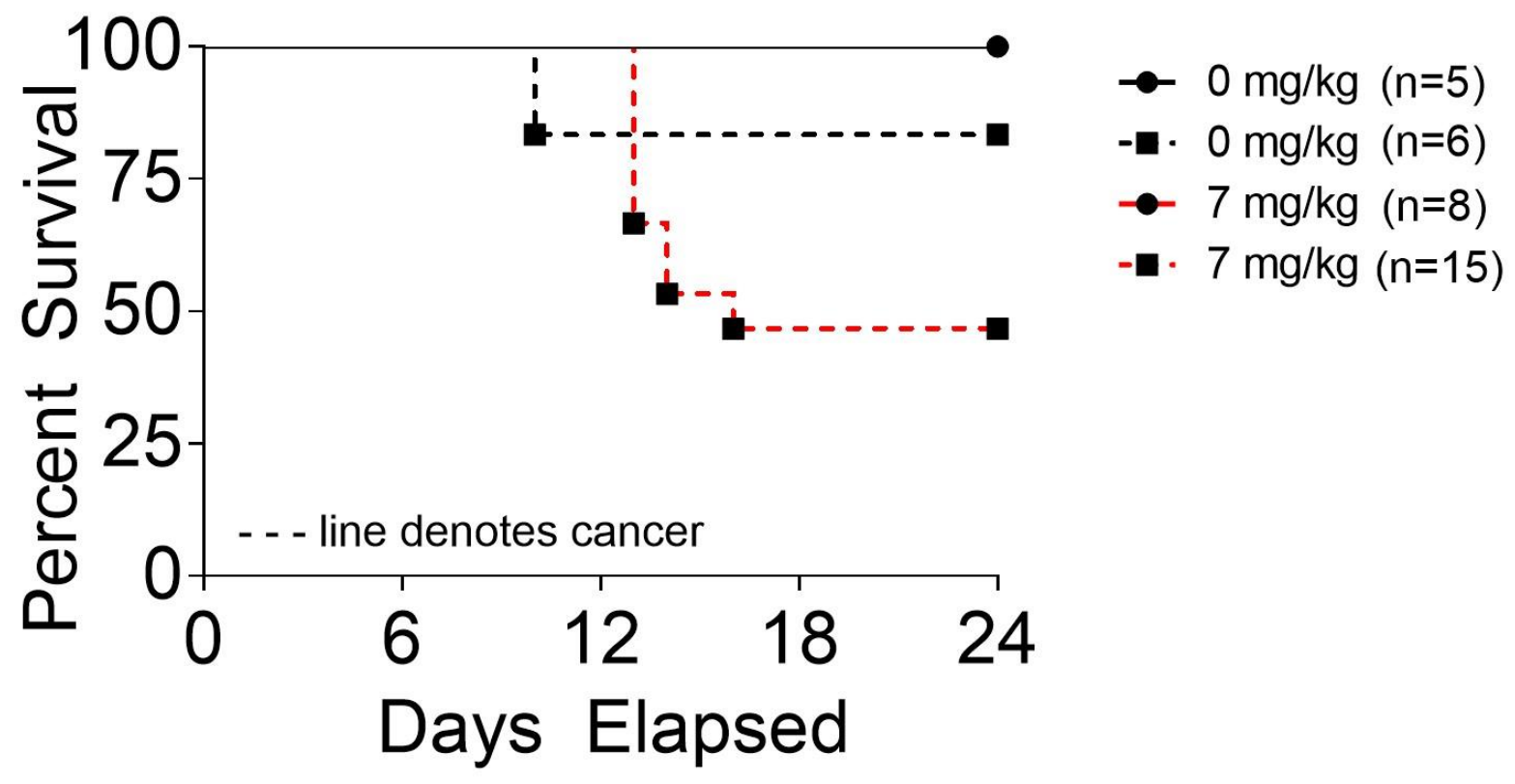

Figure 4.3. Treatment with repeated administration of cisplatin decreases overall survival in cancer mice. Forty week old male and female mice with and without cancer were treated with either 0 or $7 \mathrm{mg} / \mathrm{kg}$ cisplatin once a week for four weeks. Mice that showed obvious signs of discomfort/stress, or that had $\geq 20 \%$ weight loss were euthanized before the end of the study. 


\section{BUN and NGAL levels increase with repeated administration of cisplatin in non}

cancer and cancer mice. BUN is a standard marker of kidney function, and NGAL is an FDA-approved biomarker for kidney injury. AKI is more prevalent in cancer patients when compared to critically ill patients without cancer. Furthermore, cancer mice treated with cisplatin had worsened survival compared to non cancer mice treated with cisplatin (Fig 4.3). Therefore, we hypothesized that BUN and NGAL levels would be higher in cancer mice treated with cisplatin than in non cancer mice treated with cisplatin. BUN levels increased in both non cancer and cancer mice treated with $7 \mathrm{mg} / \mathrm{kg}$ cisplatin, but there was no difference in BUN levels between these two groups (Fig 4.4A). At Day 24, NGAL levels increased 21-fold in non cancer mice treated with cisplatin, but only 4-fold in cancer mice treated with cisplatin that survived to Day 24 (Fig 4.4B). However, NGAL levels were increased 16-fold and 76-fold at Day 6 and 13 respectively in cancer mice treated with cisplatin compared to non cancer mice treated with cisplatin (Fig 4.4C). Thus, cancer mice treated with cisplatin have earlier, worsened kidney injury compared to non cancer mice treated with the same dosing regimen. 
(A)

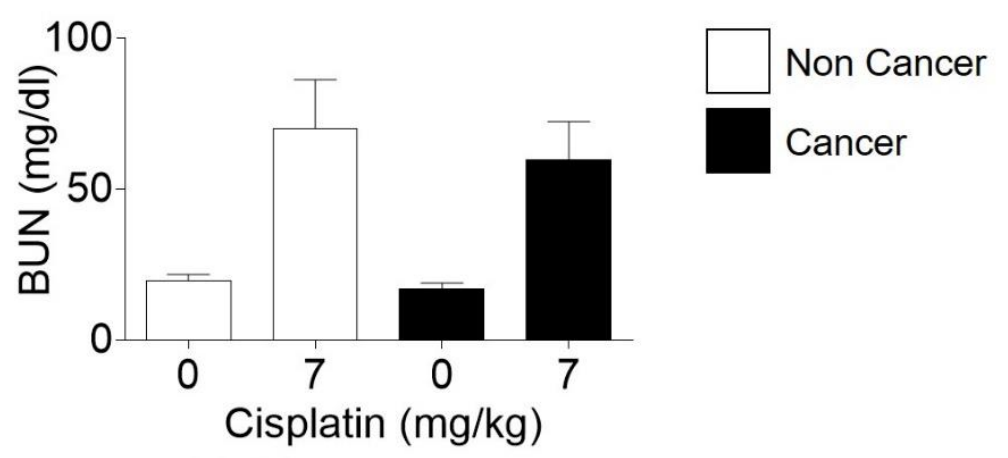

(B)

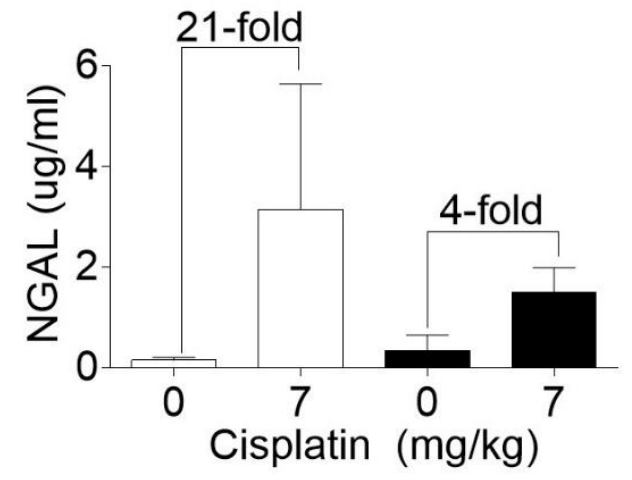

(C)

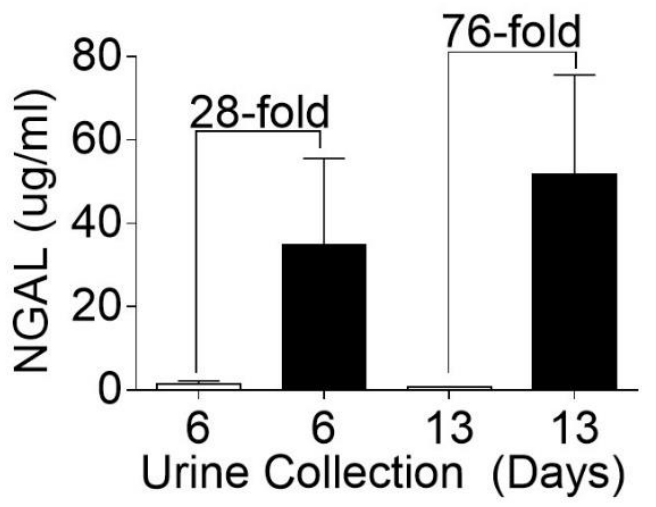

Figure 4.4. Kidney function and injury in non cancer and cancer mice treated with repeated dosing of cisplatin. Forty week old male and female mice with and without cancer were treated with either 0 or $7 \mathrm{mg} / \mathrm{kg}$ cisplatin once a week for four weeks. (A) BUN levels measured in blood plasma. (B) NGAL levels measured in urine at Day 24. (C) NGAL levels measured in urine at Day 6 and 13 in non cancer and cancer mice treated with $7 \mathrm{mg} / \mathrm{kg}$ cisplatin. Statistical significance was determined by Two-Way ANOVA followed by Tukey post-test. 
Increase in inflammatory cytokine and chemokine levels in non cancer and cancer mice treated with repeated dosing of cisplatin. Inflammation is a major pathophysiology involved in cisplatin-induced AKI and CKD. We hypothesized that since cancer mice treated with cisplatin had worsened kidney injury, that these mice would also have higher levels of inflammatory cytokines and chemokines. Tnf $\alpha, l l 6$, Mcp-1, and Cxcll were increased with cisplatin treatment in both non cancer and cancer mice (Fig 4.5A-D). Thus, cancer mice do not have a worsened inflammatory response with cisplatin treatment compared to treated non cancer mice at Day 24. 
(A)

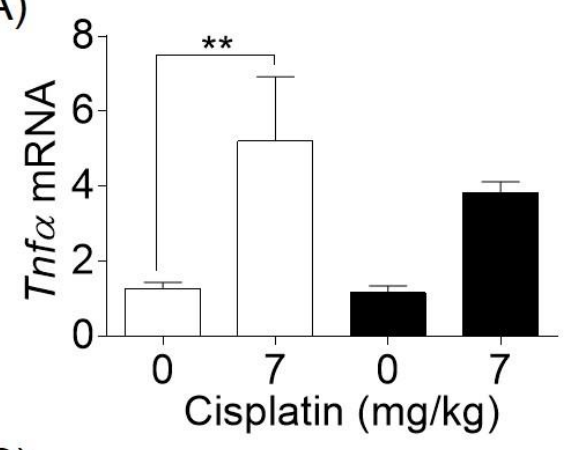

(C)

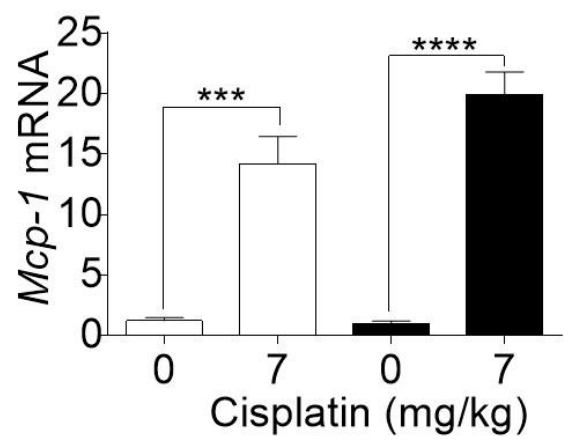

(B)

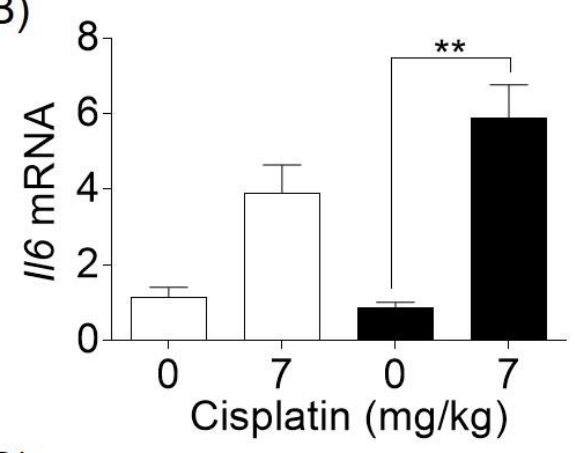

(D)

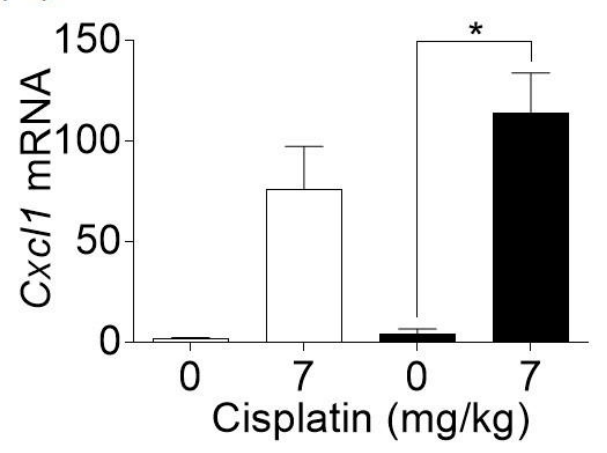

Figure 4.5. Changes in inflammatory cytokine and chemokine levels in non cancer and cancer mice treated with repeated dosing of cisplatin. Forty week old male and female mice with and without cancer were treated with either 0 or $7 \mathrm{mg} / \mathrm{kg}$ cisplatin once a week for four weeks. Levels of (A) Tnfa, (B) Il6, (C) Mcp-1, and (D) Cxcll measured in the kidney cortex via QRTPCR. Statistical significance was determined by Two-Way ANOVA followed by Tukey post-test. ${ }^{*} \mathrm{p}<0.05,{ }^{* *} \mathrm{p}<0.01,{ }^{* *} \mathrm{p}<0.001$, and $* * * * p<0.0001$. 


\section{Cancer mice develop worsened renal fibrosis when treated with the repeated dosing}

regimen of cisplatin. We have previously shown that repeated administration of low dose cisplatin induces fibrosis. TGF $\beta$ is a canonical signaling pathway in fibrogenesis, and TGF $\beta$ protein levels increased with cisplatin treatment in non cancer mice, and were further elevated in cancer mice treated with cisplatin (Fig 4.6A). Fibronectin, an ECM component, increased in the same manner (Fig 4.6A). To further confirm the presence of fibrosis, SR/FG staining for total collagen deposition and $\alpha \mathrm{SMA}$ IHC for myofibroblasts was performed. Treatment with cisplatin led to an increase in collagen deposition in non cancer mice, but levels of collagen were significantly higher in cancer mice treated with cisplatin (Fig 4.6B). Similarly, cancer mice treated with cisplatin also had increased aSMA positivity (Fig 4.6C). Taken together, these data indicate that cancer mice treated with cisplatin develop worsened renal fibrosis when compared to non cancer mice treated with the same dosing regimen. 
(A)

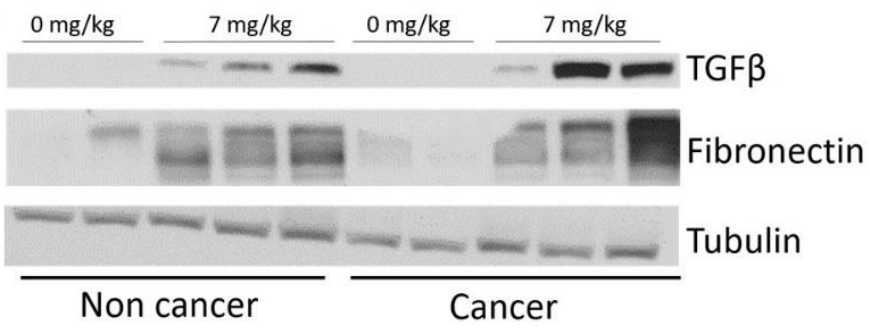

(B)

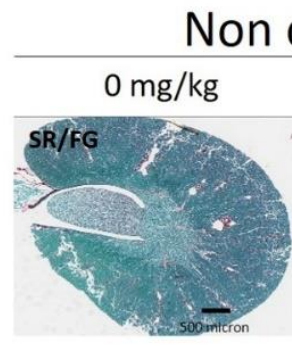

cancer

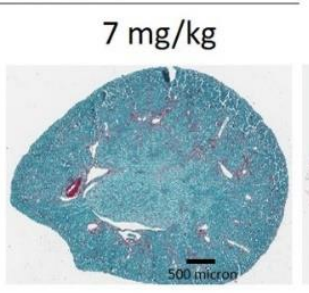

Cancer

(C)
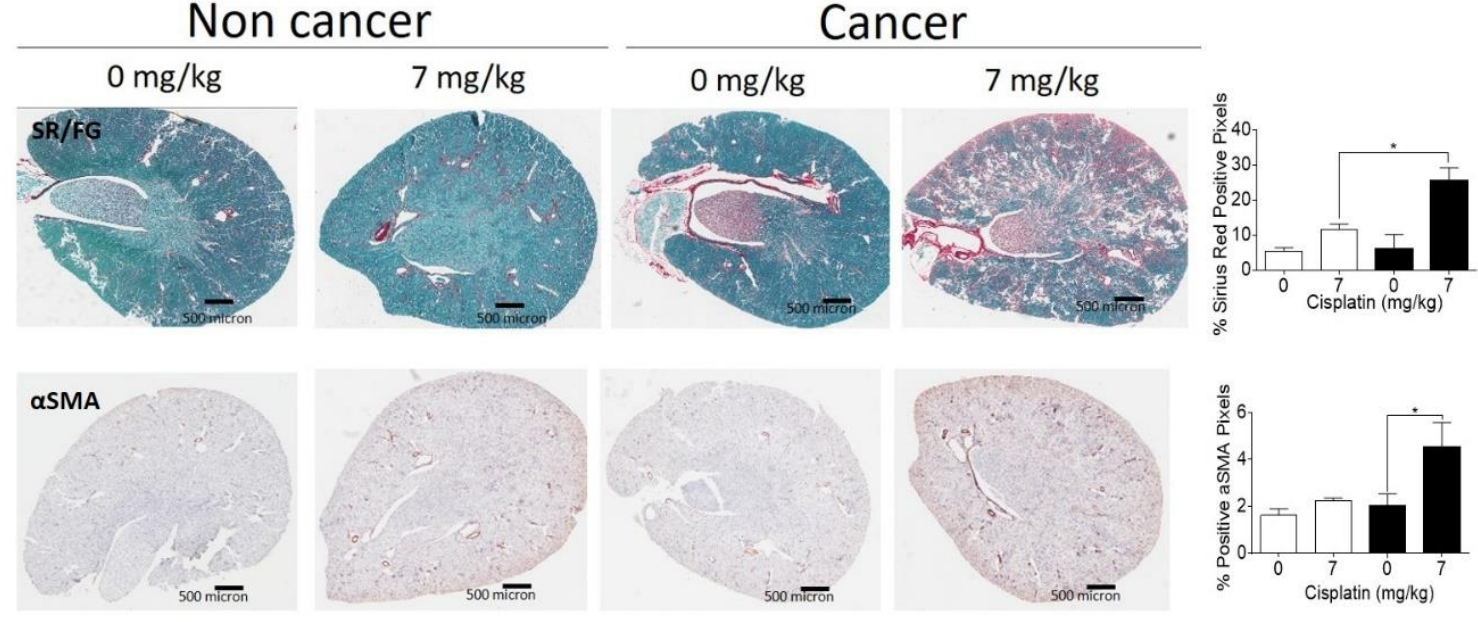

Figure 4.6. Development of fibrosis in non cancer and cancer mice treated with repeated dosing of cisplatin. Forty week old male and female mice with and without cancer were treated with either 0 or $7 \mathrm{mg} / \mathrm{kg}$ cisplatin once a week for four weeks. (A) Protein levels of TGF $\beta$ and fibronectin in kidney cortex homogenates were determined by Western blot. (B) SR/FG stain for total collagen levels and quantification of percent Sirius red positive pixels. (C) $\alpha \mathrm{SMA}$ IHC for total myofibroblasts and quantification of percent $\alpha$ SMA positive pixels. Statistical significance was determined by Two-Way ANOVA followed by Tukey post-test. 


\section{Epidermal growth factor receptor (EGFR) levels increase in cancer mice treated}

with repeated dosing of cisplatin. EGFR is upregulated and activated to increase cellular proliferation of injured proximal tubule cells. However, sustained activation of EGFR plays a role in fibrogenesis. Western blot data indicated increased EGFR protein levels in the kidneys of cancer mice treated with cisplatin (Fig 4.7A). In addition, cancer mice treated with cisplatin also had increased pEGFR Y1068 levels, indicating activation of EGFR, compared to non cancer mice treated with cisplatin (Fig 4.7A). To further confirm the differential role of EGFR in cancer mice treated with cisplatin, we utilized markers of EGFR downstream signaling pathways. Activation of JNK ( $\mathrm{pJNK}$ ) and NFKB p65 (pNFkB p 65) was higher in cancer mice treated with cisplatin than non cancer mice (Fig 4.7B). Therefore, these data indicate the role of EGFR in worsened renal fibrosis in cancer mice treated with the repeated dosing regimen of cisplatin. 


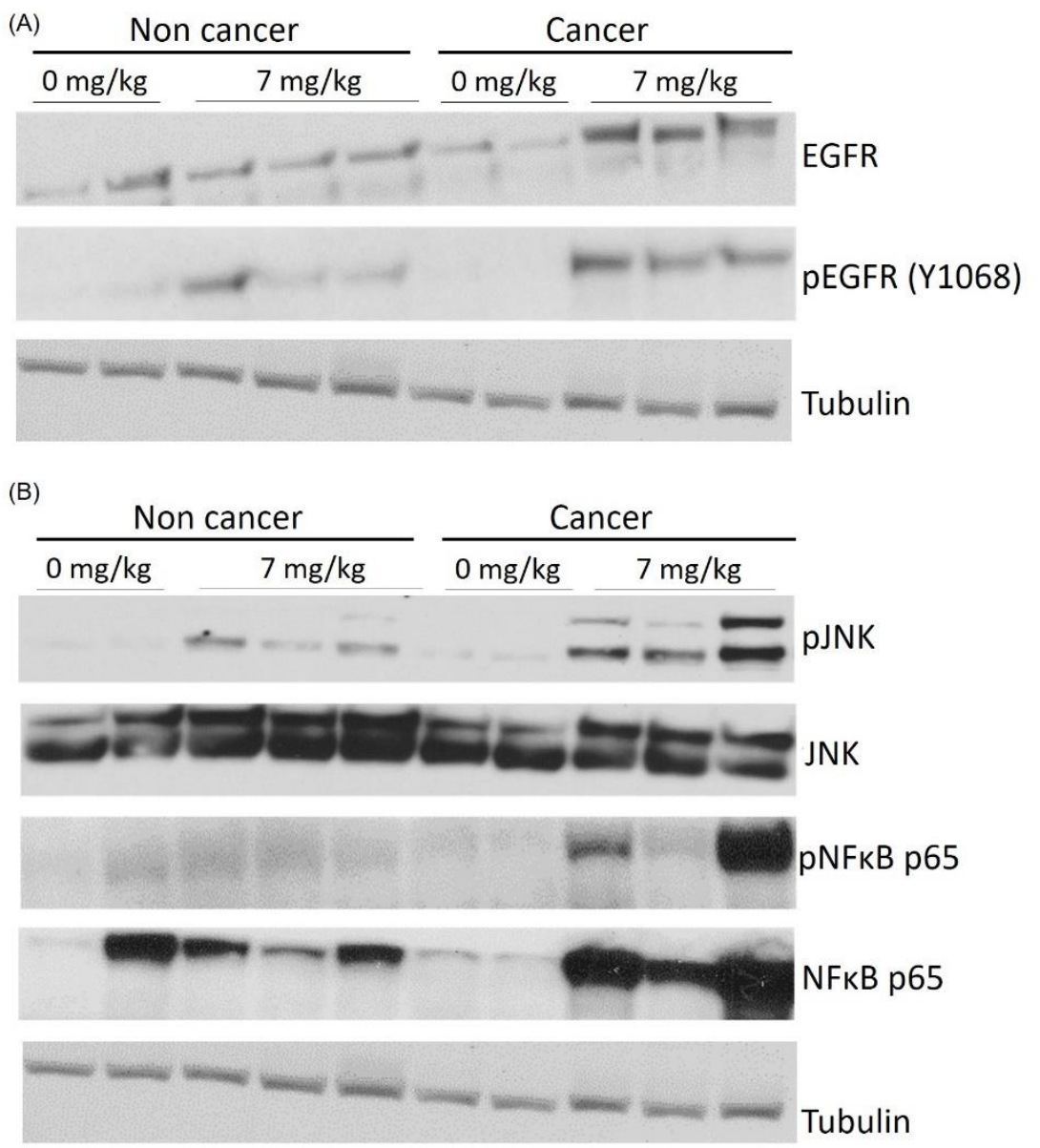

Figure 4.7. EGFR activation and downstream signaling pathway activation in non cancer and cancer mice treated with repeated dosing of cisplatin. Forty week old male and female mice with and without cancer were treated with either 0 or $7 \mathrm{mg} / \mathrm{kg}$ cisplatin once a week for four weeks. (A) Protein levels of EGFR, pEGFR (Y1068),

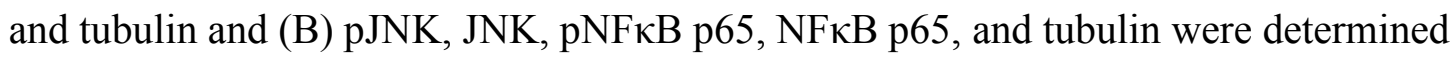
in kidney cortex homogenates were determined by Western blot. 


\section{Inhibition of EGFR leads to worsened survival and severe weight loss when mice are}

treated with cisplatin. Cancer mice treated with cisplatin had increased EGFR and subsequently increased EGFR activation (Fig 4.7A). We hypothesized that inhibition of EGFR with erlotinib in these mice would protect from the development of fibrosis, or decrease the severity of fibrosis that occurrs with cisplatin. To test this hypothesis, cohorts of non cancer and cancer mice were treated with either $0 \mathrm{mg} / \mathrm{kg}$ cisplatin and 0 $\mathrm{mg} / \mathrm{kg}$ erlotinib (V), $0 \mathrm{mg} / \mathrm{kg}$ cisplatin and $25 \mathrm{mg} / \mathrm{kg}$ erlotinib (E), $7 \mathrm{mg} / \mathrm{kg}$ cisplatin and $0 \mathrm{mg} / \mathrm{kg}$ erlotinib (C), or $7 \mathrm{mg} / \mathrm{kg}$ cisplatin and $25 \mathrm{mg} / \mathrm{kg}$ erlotinib. One dose of cisplatin and one dose of erlotinib was administered at Day 0 , followed by either 0 or $25 \mathrm{mg} / \mathrm{kg}$ erlotinib Day 1-6. Both non cancer and cancer mice treated with V, E, or C had 100\% survival to Day 7 (Fig 4.8A). However, non cancer mice treated with $\mathrm{C}+\mathrm{E}$ had $67 \%$ total survival at Day 6, and cancer mice treated with $\mathrm{C}+\mathrm{E}$ had $80 \%$ survival at Day 6 (Fig 4.8A). By Day 7, all mice had to be euthanized in the $\mathrm{C}+\mathrm{E}$ treatment groups. Weight loss in $\mathrm{C}+\mathrm{E}$ treated non cancer mice was $17 \%$, and $11 \%$ in cancer mice (Fig 4.8B). These data indicate that mice cannot survive combinatorial treatment with cisplatin and erlotinib, and this may be due to the development of AKI. 
(A)

Non cancer

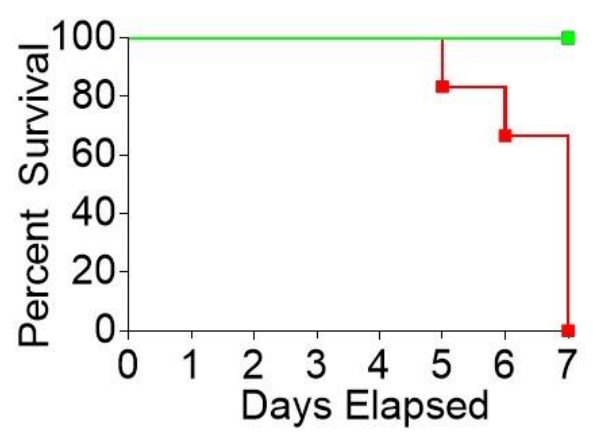

(B)

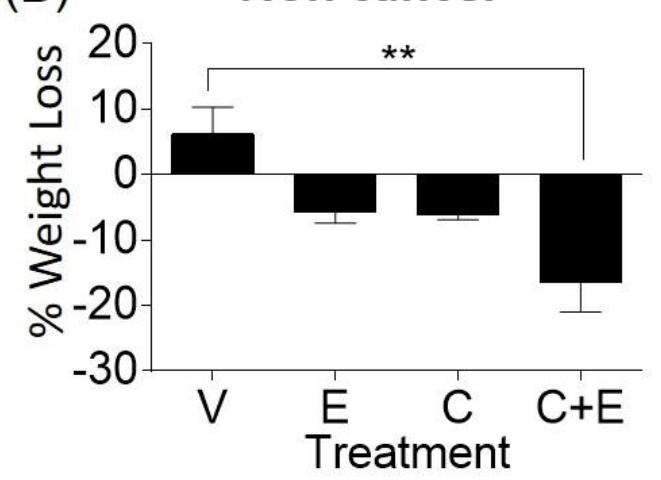

Cancer

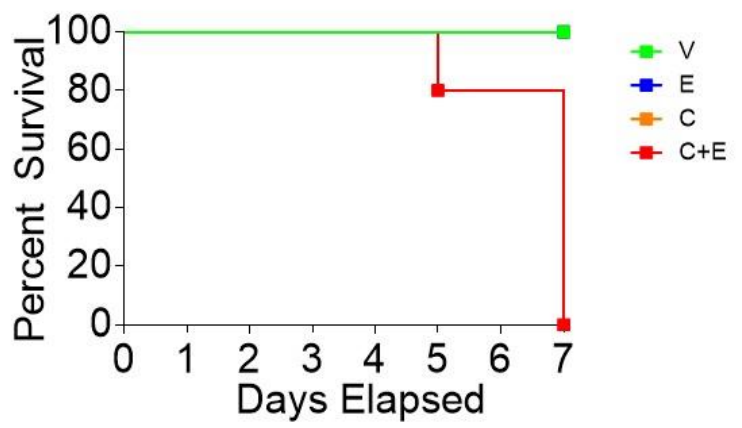

Cancer

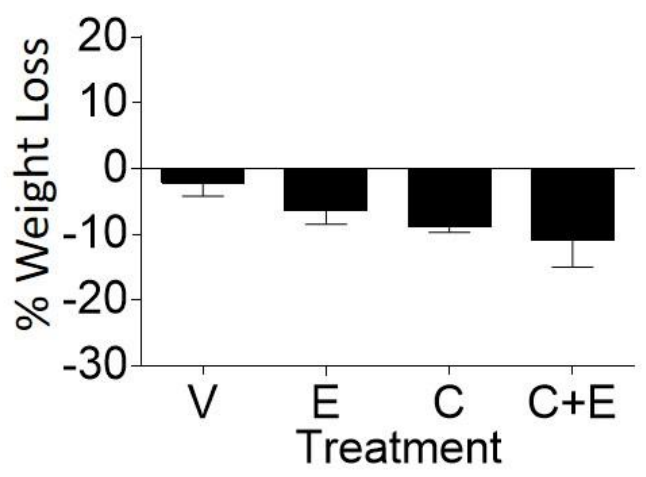

Figure 4.8. Overall survival and weight loss with cisplatin + erlotinib. Forty week old male and female mice with and without cancer were treated with either saline vehicle once (V), $25 \mathrm{mg} / \mathrm{kg}$ erlotinib once a day for 6 days (E), $7 \mathrm{mg} / \mathrm{kg}$ cisplatin once (C), or $7 \mathrm{mg} / \mathrm{kg}$ cisplatin once and $25 \mathrm{mg} / \mathrm{kg}$ erlotinib for 6 days (C+E). (A) Percent survival of mice to Day 7. (B) Total percent weight loss at Day 7. Statistical significance was determined by One-Way ANOVA followed by Tukey post-test. ${ }^{* *} \mathrm{p}<0.01$ 


\section{BUN and NGAL levels are markedly increased in non cancer and cancer mice}

treated with combinatorial cisplatin and erlotinib treatment. Based on poor survival outcomes and weight loss, we hypothesized that both cohorts of mice treated with $\mathrm{C}+\mathrm{E}$ would have increased BUN and NGAL levels, indicative of severe AKI. BUN was slightly increased with cisplatin treatment in non cancer and cancer mice, but was highly elevated in both cohorts treated with C+E (Fig 4.9A). Similarly, NGAL levels were markedly increased with $\mathrm{C}+\mathrm{E}$ treatment in both groups (Fig 4.9B). Thus, mice treated with both erlotinib and one, low dose of cisplatin develop severe AKI. 
(A)

Non cancer

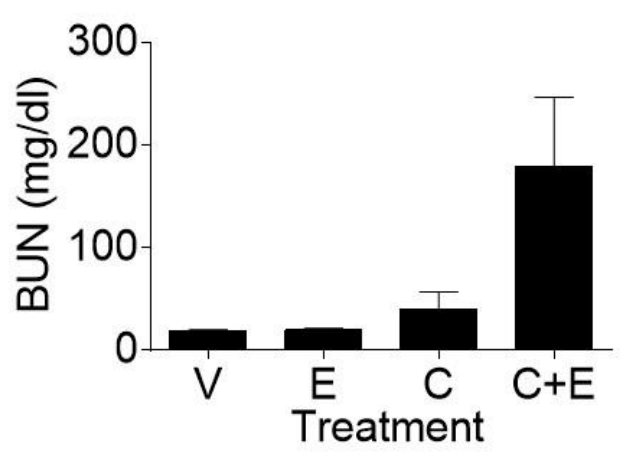

(B)

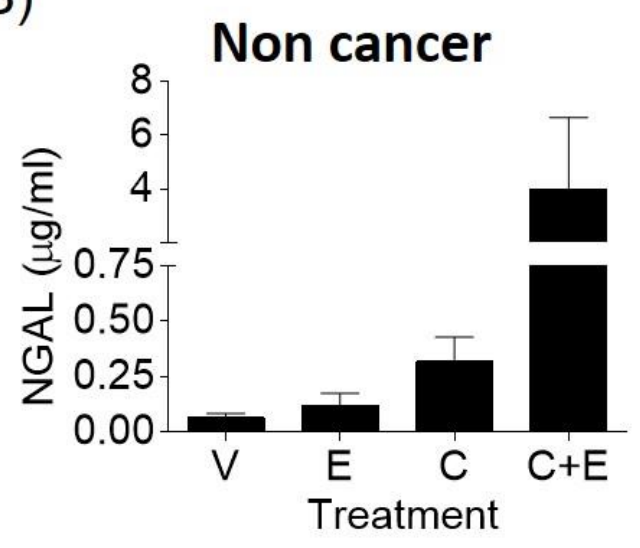

Cancer
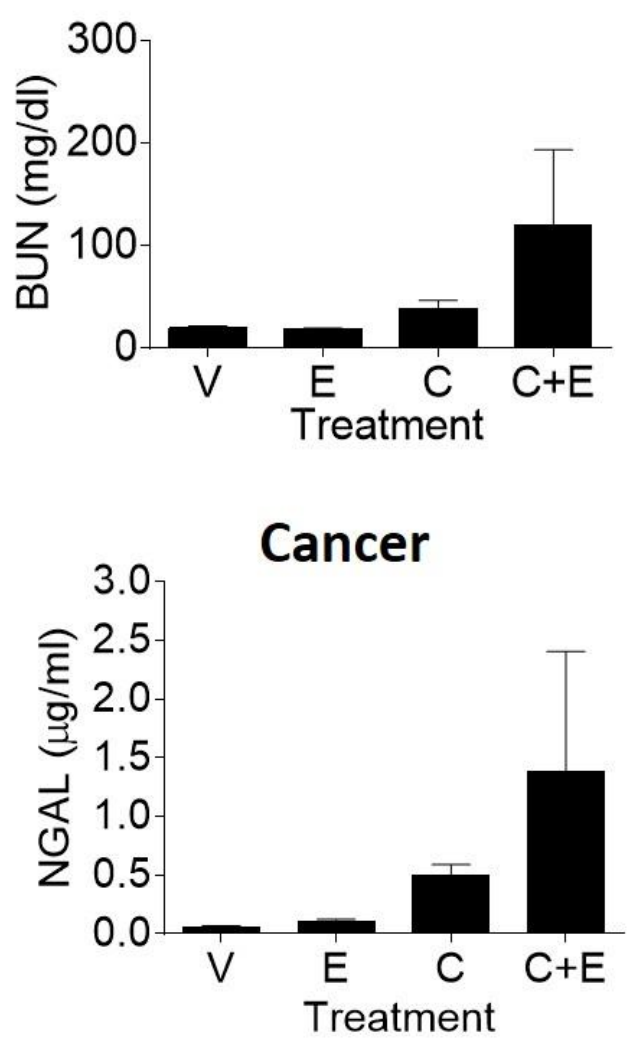

Figure 4.9. Kidney injury and loss of function with cisplatin + erlotinib. Forty week old male and female mice with and without cancer were treated with either saline vehicle once (V), $25 \mathrm{mg} / \mathrm{kg}$ erlotinib once a day for 6 days (E), $7 \mathrm{mg} / \mathrm{kg}$ cisplatin once (C), or $7 \mathrm{mg} / \mathrm{kg}$ cisplatin once and $25 \mathrm{mg} / \mathrm{kg}$ erlotinib for 6 days (C+E). (A) BUN levels measured in plasma. (B) NGAL levels measured in urine. Statistical significance was determined by One-Way ANOVA followed by Tukey post-test. 


\section{Inflammatory cytokine and chemokine levels in non cancer and cancer mice treated}

with cisplatin+erlotinib. Treatment with repeated administration of cisplatin causes an inflammatory response marked by increases in $T n f \alpha, I l 6, M c p-1$, and $C x c l-1$. In noncancer mice, $\operatorname{Tn} f \alpha$ was significantly increased with cisplatin treatment, but levels were not elevated with $\mathrm{C}+\mathrm{E}$ treatment (Fig 4.10A). $M c p-1$ and $C x c l-1$ were elevated with cisplatin (Fig 4.10B,C). Mcp-1 was slightly elevated with $\mathrm{C}+\mathrm{E}$, but $C x c l-1$ levels were increased approximately 100-fold in non cancer mice (Fig 4.10B,C). In mice with cancer, there was not an increase in $\operatorname{Tnf} \alpha$ levels in any treatment group (Fig 4.10A). However, there was a slight increase in $M c p-1$ levels with cisplatin treatment, and $C x c l-1$ was slightly elevated with $\mathrm{C}+\mathrm{E}$ treatment (Fig 4.10B,C). Additionally, non cancer and cancer mice treated with erlotinib had decreased $M c p-1$ levels. These data indicate a differential activation of inflammatory cytokines and chemokines in non cancer and cancer mice when treated with cisplatin or combinatorial $\mathrm{C}+\mathrm{E}$ treatment. 
Non Cancer

(A)

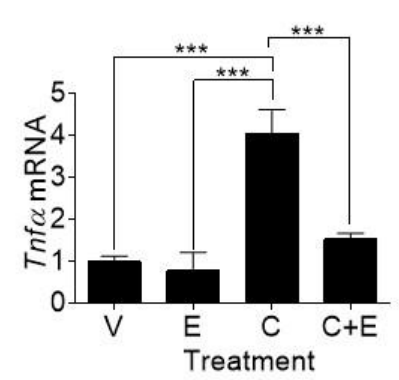

(B)

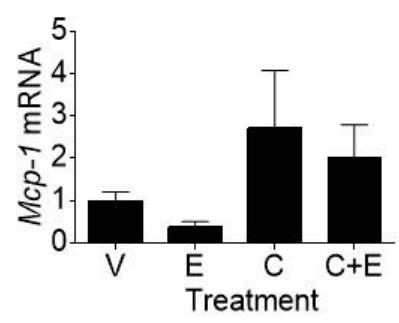

(C)

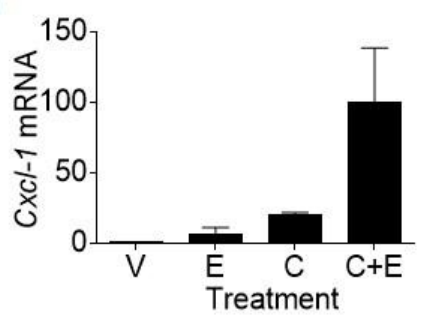

Cancer
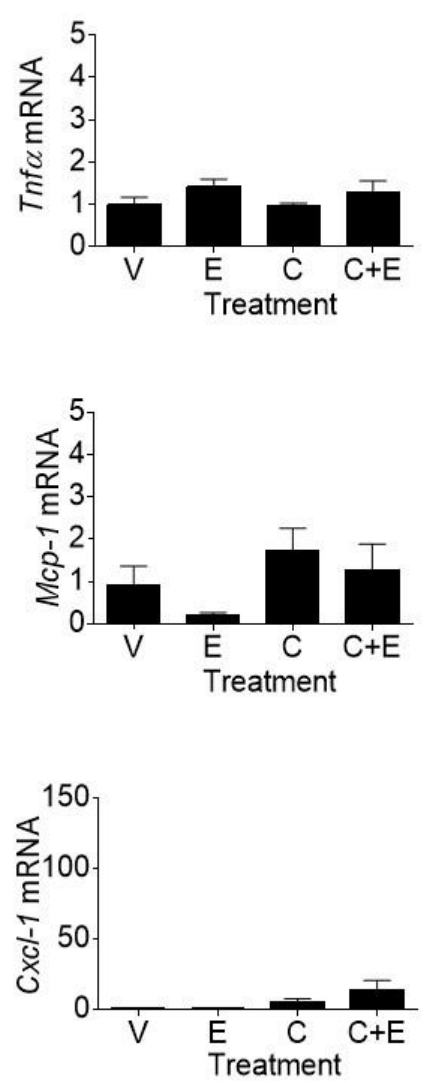

Figure 4.10. Changes in inflammatory cytokine and chemokine levels with cisplatin+erlotinib. . Forty week old male and female mice with and without cancer were treated with either saline vehicle once (V), $25 \mathrm{mg} / \mathrm{kg}$ erlotinib once a day for 6 days (E), $7 \mathrm{mg} / \mathrm{kg}$ cisplatin once (C), or $7 \mathrm{mg} / \mathrm{kg}$ cisplatin once and $25 \mathrm{mg} / \mathrm{kg}$ erlotinib for 6 days (C+E). Levels of (A) Tnf $\alpha$, (B) $M c p-1$, and (C) $C x c l-1$ were measured in the kidney via QRTPCR. Statistical significance was determined by One-Way ANOVA followed by Tukey post-test; *** indicates $\mathrm{p}<0.001$. 


\section{DISCUSSION}

Cancer patients have a higher rate of AKI compared to critically ill patients without cancer $(102,103)$. Of the causes of AKI, drug-induced nephrotoxicity is more prevalent, as only patients with cancer receive chemotherapy. Cisplatin has a high rate of nephrotoxicity in cancer patients, leading to the development of AKI in $20-30 \%$ of patients.

Studies utilizing experimental models of cancer to study the development of cisplatin nephrotoxicity are limited. Furthermore, these experiments are usually performed in xenograft or subcutaneous cancer models which do not allow for studies of cancer as a systemic disease. Mouse xenograft models are not ideal for studying the systemic effect cancer may have in relation to the development of AKI. First, tumor cells injected subcutaneously will develop at the site of injection, rather than the original site from which the cancer cells were derived. The tumor microenvironment plays a crucial role in the response of the cancer to chemotherapy, thus this relationship cannot be studied $(110,111)$. Furthermore, xenograft models that utilize human cancer cell lines require the use of immunocompromised mice for proper engraftment. A major component of cisplatin-induced kidney injury and subsequent recovery is the immune cell response $(9,10,15)$. Not being able to incorporate this essential component may affect kidney injury outcomes.

To improve on these limited xenograft studies, we utilized a transgenic mouse model of Kras4bG12D lung adenocarcinoma combined with our clinically relevant dosing regimen of cisplatin. Mice with transgenes for both Tet-o-Kras4bG12D and CCSP-rtTA develop lung adenocarcinoma in six months when doxycycline is supplied in 
the diet. This is a relevant cancer model because patients with lung cancer are often treated with repeated, lower doses of cisplatin. Furthermore, previous studies have indicated that there is lung/kidney crosstalk that may suggest a worsened kidney injury phenotype in the context of lung cancer (112). Specifically, AKI is associated with respiratory failure, prolonged ventilation, and prolonged ventilator weaning. Lung complications are associated with an increased mortality risk in the setting of AKI (112114). This is attributed to the fact that the lung contains a vast microcapillary network, making it susceptible to inflammatory cytokines and chemokines released from injured, distant organs. Furthermore, injury to the kidney may stimulate leukocytes that can exacerbate lung injury and vice versa $(113,114)$. However, the true risk of cisplatininduced kidney injury in lung cancer patients is largely unknown. The study we present here enables us to study cancer as a systemic disease, and potential effects it may have on distal organs as the result of cisplatin-induced kidney injury.

Our data indicate that mice with cancer have a higher death rate when treated with cisplatin than do age-matched non cancer mice. This seems counterintuitive as cisplatin treatment is supposed to treat cancer. However, analysis of kidney injury revealed a high peak in NGAL levels at Day 6 and 13 in cancer mice treated with cisplatin, indicative of an AKI phenotype. Mice that were able to survive to Day 24 had relatively low levels of NGAL, suggesting some mice fare better with cisplatin than others. Although we are uncertain as to why some mice with cancer die early with cisplatin treatment, we hypothesize this may due to the development of TLS or acute lung injury. Examining markers associated with these pathologies will be important in future studies. 
This study is the first to examine the development of fibrosis indicative of CKD in the context of cancer. Cancer mice treated with cisplatin had worsened fibrosis compared to non cancer mice treated with cisplatin. Taken together, this study indicates that cancer is a risk factor for the development of both cisplatin-induced AKI and subsequent CKD. This aligns with patient data in which AKI is more prevalent in patients with cancer (103).

Delving into the mechanism as to why cancer mice treated with cisplatin have worsened kidney injury outcomes, we found expression of EGFR in cancer mice treated with cisplatin was more pronounced than in non cancer mice. This differential expression of EGFR in cancer mice makes it a desirable target for treatment of cisplatininduced kidney injury. Overexpression and over-activation of EGFR have both been documented in many experimental forms of $\mathrm{AKI}$ and $\mathrm{CKD}$, including cisplatin nephrotoxicity, I/R, and folic acid nephropathy models $(74,108,109,115,116)$. However, the effects of blocking EGFR and its activation remain controversial, due to the fact that EGFR plays different roles in AKI and fibrosis.

Using a proximal tubule-specific EGFR knockout (KO) mouse in combination with the experimental I/R model, Chen et al found that although initial injury levels were comparable after surgery in both WT and KO mice, KO mice had more injury present 6 days post-surgery compared to WT mice (115). Thus, this study indicates that EGFR plays an important role in recovery after injury. However, sustained activation of EGFR can promote renal fibrosis $(74,108)$. In the angiotensin II model of renal fibrosis, EGFR is constitutively activated (108). Constitutive activation of EGFR promoted ERK signaling, which in turned led to sustained activation of TGF $\beta$, ultimately resulting in 
fibrosis. Pharmacological inhibition of EGFR decreased collagen levels and activation of TGF $\beta$ signaling (108). Thus, inhibition or KO of EGFR in the context of AKI may exacerbate injury, but be protective in the development of fibrosis.

Many studies that have examined the potential renoprotective effects of EGFR inhibition have used pharmacological inhibition with small molecule inhibitors. Of these, erlotinib is commonly used. Erlotinib is a small molecule receptor tyrosine kinase inhibitor (RTKI) with high specificity to EGFR (117). Erlotinib competes with adenosine triphosphate to inhibit EGFR autophosphorylation, thereby blocking activation of EGFR-mediated downstream signaling pathways (117). Ultimately, this culminates in $\mathrm{G}_{0} / \mathrm{G}_{1}$ cell cycle arrest, an increase in $\mathrm{p} 27$ expression, and apoptosis.

Due to its apoptotic effects, erlotinib is commonly used in the treatment of cancer, specifically in cancers that have EGFR mutations, including colon, pancreas, breast, ovarian, NSCLC, and bladder cancers (117-119). Clinical trials have shown elrotinib is also effective in cancers without EGFR mutations. Furthermore, erlotinib has commonly been tested in combination with platinum-containing chemotherapeutic regimens. In A549 lung cancer cells, erlotinib had additive effects when combined with gemcitabine, and erlotinib was synergistic with cisplatin (120). In a Phase III clinical trial, patients with NSCLC received either cisplatin+gemcitabine+placebo or cisplatin+gemcitabine+erlotinib as a first line treatment. Patients that received cisplatin+gemcitabine+erlotinib did not show a significant increase in overall survival (120). However, in a Phase III study where erlotinib was used as a maintenance treatment after platinum-doublet chemotherapy, patients with and without EGFR 
overexpressing cancers showed a $40.8 \% 12$ week disease control rate, compared to the placebo group (27.4\% 12 week disease control rate) (118).

The potential anti-tumor efficacy of erlotinib with other chemotherapeutics, especially cisplatin, makes it an even more attractive target for its use as a renoprotective agent. In a study by Wada $e t a l$, rats were treated with one dose of erlotinib and cisplatin to determine if erlotinib was renoprotective. By Day 4, rats treated with erlotinib and cisplatin had better renal function and decreased tubulointerstitial injury compared to rats that received cisplatin alone (121). In addition, erlotinib and cisplatin combinatorial treatment caused less apoptosis and a decrease in proliferating cells, indicative of less renal injury. Erlotinib and cisplatin treatment also decreased mRNA expression levels of profibrogenic markers, specifically TGF $\beta$ (121). However, erlotinib in combination with cisplatin had no overall effect on inflammatory cytokine expression, or immune cell infiltration.

While the study by Wada et al highlights the promise of erlotinib as a renoprotective agent, our study indicates that administration of erlotinib with cisplatin exacerbates nephrotoxicity and causes severe AKI compared to mice treated with cisplatin alone. Combinatorial treatment of cisplatin+erlotinib caused severe kidney injury and loss of function. Our data coincides with clinical data that has shown that patients treated with cisplatin+gemcitabine+erlotinib have an increase in renal failure compared to patients who receive chemotherapy without erlotinib, although increased renal failure was attributed to insufficient hydration caused by erlotinib-associated diarrhea (120). Other studies have indicated that small molecule receptor tyrosine kinase inhibitors (RTKIs) have a serious impact on electrolyte balance which can contribute to 
renal injury (122). In an FDA toxicity study, there were 63 reported cases of renal impairment with erlotinib, and the most common renal side effects included hypertension, hyponatremia, and hypokalemia (123). Furthermore, Dimke et al found that treating mice with high dose erlotinib for 23 days caused a decrease in $\mathrm{Mg}^{2+}$ serum levels associated with altered magnesium handling (124). In a clinical trial examining the toxic profile of erlotinib and sorafenib (a VEGFR-2 inhibitor), 34\% of patients developed hypophosphatemia compared to treatment with sorafenib alone (119).

Although it is unclear how erlotinib causes these electrolyte imbalances, we hypothesize that cisplatin+erlotinib may cause severe AKI via damage to proximal tubule cells in combination with electrolyte imbalance. While we did not measure electrolyte levels in the serum in this study, this is a necessary and important follow-up study. Furthermore, while our study indicates that erlotinib is not a good choice as a renoprotective agent in cisplatin-induced kidney injury, it may have some utility as an injury ameliorating drug in our model. Western blots indicated an increase in EGFR and pEGFR (Y1068) at Day 24 in cancer mice treated with cisplatin, suggesting there may be sustained activation of EGFR. Since sustained activation of EGFR promotes renal fibrosis, treating mice with erlotinib once fibrotic processes are already activated may hinder further injury or ameliorate fibrosis that has already developed.

Taken together, our data highlight the importance of studying cisplatin-induced kidney injury by utilizing a cancer mouse model. Cancer mice have worsened kidney outcomes compared to non cancer mice treated with our repeated dosing regimen of cisplatin. Our data recapitulates clinical data that indicates there is a greater rate of AKI in cancer patients than in critically ill, non cancer patients. While this statistic may be 
due to the fact that cancer patients are more likely to be exposed to kidney insults, our data suggests that, at least in the context of lung cancer, there may be an interaction between the kidney and cancer that exacerbates kidney injury. 


\section{CHAPTER 5}

\section{OVERALL SUMMARY}

Cisplatin has been a mainstay in the treatment of many solid tumor cancers either alone or in combination with surgery, radiation, or other chemotherapeutics since the 1970's (2). While cisplatin is a potent therapeutic for treating cancer, its dose-limiting side effect of nephrotoxicity causes AKI in $20-30 \%$ of patients. AKI itself is a risk factor for the development of CKD, a progressive disease marked by the development of renal fibrosis $(26,57)$. Both $\mathrm{AKI}$ and $\mathrm{CKD}$ often require dialysis and are associated with an increased mortality risk, however, there are no pharmacological interventions for either form of kidney injury $(9,27,57)$. For cisplatin-induced kidney injury specifically, we believe that this deficit in therapeutic approaches is because of the lack of a relevant experimental mouse model of cisplatin nephrotoxicity. Thus, the overall goal of this dissertation was to develop a clinically relevant mouse model of cisplatin-induced kidney injury in hopes of identifying novel therapeutic targets for the prevention or treatment of the disease.

To develop this clinically relevant model, we had two main objectives: (1) develop a dosing regimen of cisplatin that recapitulates how patients receive cisplatin, and (2) include aging and cancer, as these are pertinent risk factors for AKI. Our work and the work of others have shown that low dose, repeated administration of cisplatin induces renal fibrosis with minimal cell death. While fibrosis is indicative of CKD, we 
wanted to determine if it was possible to develop bona fide CKD after cisplatin-induced AKI. Thus, we treated mice with our repeated dosing regimen of cisplatin $(7 \mathrm{mg} / \mathrm{kg}$ cisplatin once a week for four weeks) and allowed mice to age for 6 months posttreatment. At this time point, mice had progressive interstitial fibrosis and inflammation, persistent endothelial and tubular injury, and the development of glomerular pathologies that contributed to a significant decline in renal function (Table 5.1). Thus, this work validates the notion that $\mathrm{AKI}$ can progress to $\mathrm{CKD}$, and that only mild, sub-clinical injury is necessary for the development of fibrosis. Our work indicates that more patients treated with cisplatin may be at risk for developing poor long-term kidney outcomes than previously thought. 


\begin{tabular}{|c|c|c|c|}
\hline & Dose 2 & Dose 4 & 6 months post-treatment \\
\hline BUN & No Change & Slight increase & Significant increase \\
\hline NGAL & Slight increase & Significant increase & Return to baseline \\
\hline $\begin{array}{l}\text { Inflammation/ Immune cell } \\
\text { infiltration }\end{array}$ & $\begin{array}{l}\text { Tnfo, II6, Mcp-1, and Cxcl1 } \\
\text { levels increase slightly; no } \\
\text { macrophage infiltration }\end{array}$ & $\begin{array}{l}\text { Tnfa significantly increases, } \\
\text { II6 remains slightly elevated, } \\
\text { and } \mathrm{Cxcl} 1 \text { significantly } \\
\text { increases; mild macrophage } \\
\text { infiltration }\end{array}$ & $\begin{array}{l}\text { Tnfa remains elevated and } \\
1 / 6 \text { significantly increases; } \\
\text { severe macrophage } \\
\text { infiltration }\end{array}$ \\
\hline Tubular Damage & Slight damage & Severe damage & $\begin{array}{l}\text { Persistent damage; distal } \\
\text { damage }\end{array}$ \\
\hline Endothelial Dysfunction & $\begin{array}{l}\text { Slight elevation of } \\
\text { endothelin-1, Icam-1, Vcam- } \\
\text { 1, Timp-1 }\end{array}$ & $\begin{array}{l}\text { Significant elevation of } \\
\text { endothelin-1, Icam-1, Vcam- } \\
\text { 1, and Timp-1 }\end{array}$ & $\begin{array}{l}\text { Persistent endothelial } \\
\text { dysfunction; cells in medulla } \\
\text { overexpress CD31 indicative } \\
\text { of atherosclerosis }\end{array}$ \\
\hline Fibrosis & Not present & $\begin{array}{l}\text { Pro-fibrotic growth factor } \\
\text { levels increase; fibrosis } \\
\text { present }\end{array}$ & $\begin{array}{l}\text { Pro-fibrotic growth factor } \\
\text { levels remain elevated; } \\
\text { fibrosis is progressive }\end{array}$ \\
\hline Glomerular Damage & Mesangial hypercellularity & Mesangial hypercellularity & $\begin{array}{l}\text { Mesangial hypercellularity, } \\
\text { periglomerular fibrosis, and } \\
\text { thickening of glomerular } \\
\text { basement membrane }\end{array}$ \\
\hline
\end{tabular}

Table 5.1. Overview of injury with the repeated dosing regimen of cisplatin.

After Dose 2 of cisplatin, there is minimal damage to the kidney. However, after

Dose 4 there is severe endothelial dysfunction and tubular damage, and fibrosis

develops. Six months after treatment, inflammation and fibrosis are worsened, and glomerular pathologies indicative of CKD.

Furthermore, it has been hypothesized that the risk of developing certain forms of AKI increases with aging. The median age of all cancer diagnoses is 65 , and therefore this population may be at risk for developing exacerbated cisplatin-induced kidney injury, although previous patient and experimental data have been unclear about what the true risk is. Combining our clinically relevant dosing regimen and aging, we have provided a comprehensive study of aging and cisplatin nephrotoxicity. Our data indicate that aged mice do not develop worsened AKI or fibrosis indicative of CKD compared to young mice (Table 5.2A,B). However, aged mice have higher levels of collagen deposition, 
myofibroblasts, and injury basally in the kidney (Table 5.2B). Furthermore, while repeated administration of cisplatin in aged mice leads to increased immune cell infiltration, there was less of an inflammatory cytokine response, and fibrosis was not exacerbated (Table 5.2). This lack of difference between young and aged mice in terms of injury may suggest that age is not as big of a patient risk factor for cisplatin nephrotoxicity as initially believed. 
(A)

\begin{tabular}{|c|c|c|}
\hline $\begin{array}{l}\text { Standard dosing model of cisplatin } \\
\text { (AKI) }\end{array}$ & Young mice & Aged mice \\
\hline Survival & $100 \%$ survival & $\begin{array}{l}80 \% \text { survival in mice treated with } \\
\text { cisplatin }\end{array}$ \\
\hline Weight Loss & $\begin{array}{l}17.4 \% \text { average weight loss with } \\
\text { cisplatin }\end{array}$ & $\begin{array}{l}11.9 \% \text { average weight loss with } \\
\text { cisplatin }\end{array}$ \\
\hline BUN & Significant increase & Significant increase \\
\hline NGAL & 15000 -fold increase with cisplatin & $\begin{array}{l}\text { 15-fold increase with cisplatin; } \\
\text { NGAL basally elevated }\end{array}$ \\
\hline Inflammation & $\begin{array}{l}\text { Significant increase in } \operatorname{Tnf} \alpha, 116 \text {, } \\
C x c l-1 \text {, and } M c p-1 \text { levels }\end{array}$ & $\begin{array}{l}\text { Tnf } \alpha \text { and } / / 6 \text { slightly elevated; } C x c l-1 \\
\text { significantly increased and } M c p-1 \\
\text { elevated }\end{array}$ \\
\hline Cell Stress/Cell Death & $\begin{array}{l}\mathrm{CHOP} \text { and } \mathrm{CC} 3 \text { elevated with } \\
\text { cisplatin }\end{array}$ & $\begin{array}{l}\text { CHOP basally elevated; } \mathrm{CHOP} \text { and } \\
\mathrm{CC} 3 \text { elevated with cisplatin }\end{array}$ \\
\hline $\begin{array}{l}\text { Repeated Dosing Model of } \\
\text { Cisplatin }\end{array}$ & Young mice & Aged mice \\
\hline Survival & $100 \%$ survival & $100 \%$ survival \\
\hline Weight Loss & $<10 \%$ weight loss with cisplatin & $<10 \%$ weight loss with cisplatin \\
\hline BUN & Slight increase & Slight increase \\
\hline NGAL & 51.2-fold increase with cisplatin & $\begin{array}{l}\text { NGAL basally elevated; 9.3-fold } \\
\text { increase }\end{array}$ \\
\hline Inflammation & $\begin{array}{l}\text { Tnfo, II6, Mcp-1, and Cxcl-1 levels } \\
\text { significantly increased }\end{array}$ & $\begin{array}{l}\text { Tnfo and } / 16 \text { slightly elevated; Mcp- } \\
1 \text { and } C x c l-1 \text { levels significantly } \\
\text { increased }\end{array}$ \\
\hline Fibrosis & Fibrosis present with cisplatin & $\begin{array}{l}\text { Collagen levels and myofibroblasts } \\
\text { basally elevated; minimal increase } \\
\text { with cisplatin treatment }\end{array}$ \\
\hline Immune Cell Infiltration & $\begin{array}{l}\mathrm{F} 4 / 80+\text { macrophages and } \mathrm{CD} 3+\mathrm{T} \\
\text { cells present }\end{array}$ & $\begin{array}{l}\text { More } \mathrm{CD} 3+\mathrm{T} \text { cells and } \mathrm{F} 4 / 80+ \\
\text { macrophages present; } \mathrm{CD} 3+\mathrm{T} \text { cells } \\
\text { form clusters }\end{array}$ \\
\hline
\end{tabular}

Table 5.2. Differences between 8 and 40 week old mice treated with either the standard dosing or repeated dosing regimen of cisplatin. (A) With the standard dosing regimen of cisplatin, aged mice have a decreased overall survival, and less of an inflammatory response. (B) With repeated dosing, collagen levels and myofibroblasts are basally elevated in 40 week old mice, but these mice do not develop worsened fibrosis compared to 8 week old mice treated with cisplatin. In addition, aged mice have increased immune cell infiltration. 
In addition to the incorporating aging into our model, it was important to also include cancer. Cancer patients have a greater rate of developing AKI in general than critically ill patients without cancer, and only patients with cancer receive cisplatin. Using a transgenic mouse model of lung adenocarcinoma in combination with our repeated dosing regimen of cisplatin, we found that cancer mice had decreased survival, increased kidney injury, and worsened renal fibrosis compared to non cancer mice treated with the same dosing regimen (Table 5.3A). Further analyses revealed an upregulation of EGFR and pEGFR Y1068 in cancer mice treated with cisplatin, making EGFR a good potential therapeutic target. However, administration of erlotinib, an FDA-approved EGFR inhibitor, in combination with cisplatin caused severe AKI (Table 5.3B). Thus, erlotinib was not a viable renoprotective agent. 
(A)

\begin{tabular}{|c|c|c|}
\hline $\begin{array}{l}\text { Repeated Dosing Regimen of } \\
\text { Cisplatin }\end{array}$ & Non cancer mice & Cancer mice \\
\hline Survival & $100 \%$ survival & $\begin{array}{l}83 \% \text { survival with vehicle, }<50 \% \\
\text { survival with cisplatin }\end{array}$ \\
\hline BUN & BUN levels increased & BUN levels increased \\
\hline NGAL & 21-fold increase at Day 24 & $\begin{array}{l}\text { 4-fold increase at Day } 24 \text {, but NGAL } \\
\text { levels very high after D1 and D2 } \\
\text { compared to non cancer mice }\end{array}$ \\
\hline Inflammation & $\begin{array}{l}\text { Tnf } \alpha \text { and } M c p-1 \text { levels significantly } \\
\text { increased; } 1 / 6 \text { and } C x c /-1 \text { elevated }\end{array}$ & $\begin{array}{l}\text { II6, Mcp-1, and } C x c l-1 \text { level } \\
\text { significantly increased; Tnf } \alpha \\
\text { elevated }\end{array}$ \\
\hline Fibrosis & Slight fibrosis & Exacerbated fibrosis \\
\hline $\begin{array}{l}\text { Combinatorial Treatment with } \\
\text { Cisplatin + Erlotinib }\end{array}$ & Non cancer mice & Cancer mice \\
\hline Survival & $67 \%$ survival to Day 7 & $80 \%$ survival to Day 7 \\
\hline Weight Loss & $\begin{array}{l}17 \% \text { weight loss with } \\
\text { cisplatin+erlotinib }\end{array}$ & $\begin{array}{l}11 \% \text { weight loss with } \\
\text { cisplatin+erlotinib }\end{array}$ \\
\hline BUN & BUN highly elevated & BUN highly elevated \\
\hline NGAL & NGAL highly elevated & NGAL highly elevated \\
\hline Inflammation & $\begin{array}{l}\text { Cxcl-1 highly elevated with } \\
\text { cisplatin+erlotinib }\end{array}$ & $\begin{array}{l}\text { Cxcl-1 slightly elevated with } \\
\text { cisplatin+erlotinib }\end{array}$ \\
\hline
\end{tabular}

Table 5.3. Differences in injury of non cancer and cancer mice treated with the repeated dosing regimen of cisplatin. (A) Mice with cancer have decreased overall survival to Day 24 and higher levels of NGAL earlier with cisplatin treatment compared to non cancer mice. Cancer mice treated with cisplatin that survive to Day 24 also have worsened fibrosis. (B) Combinatorial treatment with cisplatin + erlotinib causes severe AKI in both non cancer and cancer mice.

In total, work outlined in this dissertation highlights the need for designing clinically relevant animal models of disease. Using our newly established mouse model of cisplatin-induced kidney injury, we hope to identify novel therapeutic targets for treating $\mathrm{AKI} / \mathrm{CKD}$, as well as identifying potential biomarkers for monitoring disease progression. Furthermore, utilization of this model to test already developed therapeutics 
for the treatment of $\mathrm{AKI} / \mathrm{CKD}$ will hopefully lead to better results once these drugs are put into clinical trials.

\section{STRENGTHS/LIMITATIONS}

\section{STRENGTHS}

The work presented here is, to our knowledge, the first comprehensive experimental study of cisplatin-induced kidney injury leading to CKD. Previous work in this field has focused on AKI using a non-physiologically relevant mouse model of cisplatin nephrotoxicity. Data published with this standard model of cisplatin nephrotoxicity have indicated that targeting apoptosis or necrosis is important for protecting against AKI. However, pharmacological inhibitors of apoptosis have not fared well in clinical trials. We have demonstrated that when a more clinically relevant dosing regimen of cisplatin is used, there is minimal cell death. Thus, our data challenges the field of cisplatin-induced AKI research to seek out alternative therapies for the prevention of nephrotoxicity- namely targeting fibrotic pathways instead of cell death pathways.

Our work also challenges the notion that aging is a risk factor for worsened kidney injury with cisplatin treatment. Data in this field is rather limited, and most of the work focuses on the development of AKI. However, our data indicate that in both the standard model of cisplatin-induced AKI and our newly developed model in which mice develop fibrosis indicative of CKD, that middle-aged mice do not have worsened kidney outcomes when treated with cisplatin. This is particularly interesting because aged mice do have kidney injury, higher collagen levels, and more myofibroblasts present basally in comparison to untreated, young mice. We believe our work may suggest that aged mice 
are somewhat protected from the development of kidney injury, although we do not know the mechanism by which this is occurring.

Finally, we are the first to demonstrate that mice with cancer treated with our clinically relevant dosing regimen of cisplatin develop more severe kidney injury and fibrosis compared to non cancer mice treated with cisplatin. Our novel approach was to use a transgenic mouse model of lung adenocarcinoma, which is preferable to xenograft models. Utilizing a transgenic model of cancer enables us to study cancer as a systemic disease and truly understand the effect cancer itself may have on distal organs, particularly the kidney. Furthermore, our data with this cancer model suggest that cancer is a risk factor for kidney injury. This recapitulates human data studies where patients with cancer have a higher rate of AKI than non cancer, critically ill patients. Incorporation of cancer with repeated, low dose administration of cisplatin is the crux of making the model presented in this dissertation translationally relevant. We have further demonstrated this point by identifying EGFR as a therapeutic target for protecting against the development of fibrosis. However, inhibition of EGFR with erlotinib caused severe AKI. Again, this recapitulates data from clinical trials that have shown erlotinib in combination with other chemotherapeutics or targeted therapies can cause electrolyte imbalances which can contribute to functional loss of the kidney.

\section{LIMITATIONS}

While we believe that our model has the potential to be a paradigm shift in the way we study and think about cisplatin-induced kidney injury, there are several limitations associated with the model. First, we do not know how the dose given to mice compares to the clinical dose given to humans. Even when body surface area is taken into account, it 
is difficult to calculate dose equivalencies between mice and humans. This is further hindered by a difference in cisplatin clearance. Mice have 2.4-20 times greater plasma concentration of cisplatin than humans, but the half-life of cisplatin is also shorter in mice (125). Thus, there is a faster distribution of cisplatin to tissues in mice, suggesting mice may be more susceptible to nephrotoxicity than humans. Regardless, our repeated dosing regimen combined with dosing regimen of others, regardless of dose, all indicate that fibrosis is the result of repeated administration of cisplatin.

While our model indicates that fibrosis, not cell death, is the result of repeated administration of cisplatin, very little clinical data exists to indicate that patients also develop fibrosis with cisplatin alone. The lack of data is due to the fact that many patients have comorbidities that may predispose them to CKD and the development of fibrosis, namely cardiovascular disease or diabetes regardless of cisplatin treatment. In addition, kidney biopsies are not routinely taken during the course of cisplatin treatment. In fact, we tried to utilize kidney biopsy banks from O'Brien centers across the U.S. and were unable to find any samples from patients who did not also have diabetes or other comorbidities.

Finally, our model utilizes FVB/n background mice, but most renal studies are performed in $\mathrm{C} 57 \mathrm{BL} / 6 \mathrm{~J}$ mice. $\mathrm{C} 57 \mathrm{BL} / 6 \mathrm{~J}$ mice are known to be resistant to some forms of renal fibrosis. In a model of Type 1 diabetic nephropathy utilizing streptozocin, C57BL/6J mice did not develop interstitial fibrosis (126). In a model of BSA-induced renal fibrosis, C57BL/6J mice had significantly lower levels of collagen type 1 expression, a major component of extracellular matrix produced during fibrosis $(59,127,128)$. In fact, treating C57BL/6J mice with $7 \mathrm{mg} / \mathrm{kg}$ cisplatin once a week for four weeks (repeated dosing regimen) does not result in fibrosis. However, preliminary data from our lab indicates that 
increasing the dose to $9 \mathrm{mg} / \mathrm{kg}$ is sufficient to induce a similar level of fibrosis to what FVB/n mice develop with $7 \mathrm{mg} / \mathrm{kg}$ (Fig 5.1). Thus, the utility of our model is flexible by means of adjusting the dosage used in other strains of mice.

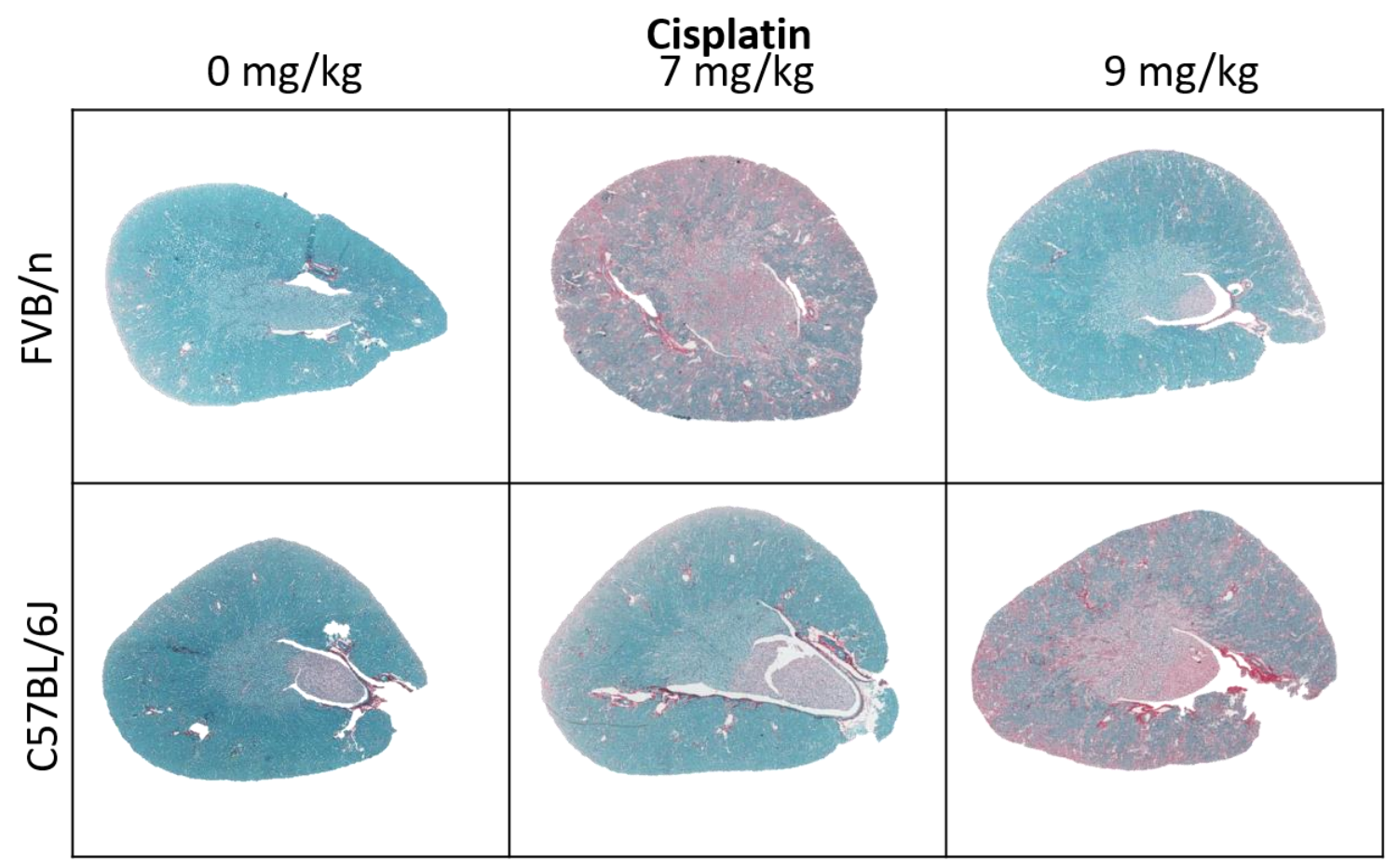

Figure 5.1. Development of renal fibrosis in FVB/n and C57BL/6J mice.

C57BL/6J mice are known to be resistant to the development of renal fibrosis in many experimental models of kidney injury. FVB/n mice develop interstitial fibrosis with repeated dosing of $7 \mathrm{mg} / \mathrm{kg}$ cisplatin, but C57BL/6J do not develop fibrosis with this dose. However, increasing the dose to $9 \mathrm{mg} / \mathrm{kg}$ results in fibrosis in C57BL/6J mice.

\section{FUTURE DIRECTIONS}

While we have worked extensively to characterize and validate our clinically relevant mouse model of cisplatin-induced kidney injury, the mechanisms underlying some of our results have yet to be elucidated. The future directions are outlined below: 


\section{Understanding the role of immune cells in the development of renal fibrosis.}

Immune cells play an essential role in kidney injury, and we have shown that increased infiltration of macrophages into the kidney is associated with the development of fibrosis. However, in the context of aging, immune cell infiltration does not necessarily indicate that injury or fibrosis will be worse. In fact, despite more immune cell infiltration of macrophages and T cells in the kidneys of aged mice treated with repeated dosing of cisplatin, the increase in collagen levels is rather low. In this way, it might be interpreted that aged mice may be protected from the development of fibrosis due to an immunosuppressive phenotype as evidenced by less of an inflammatory response with cisplatin treatment. In order to gain further insight into this hypothesis, it would be interesting to replace bone marrow in aged mice with bone marrow from young mice and vice versa, thereby giving these mice a different immune system. We would expect that if immunosuppression protects from the development of fibrosis that young mice with aged mouse bone marrow would have less fibrosis. Additionally, aged mice with young bone marrow would develop more fibrosis. This would challenge the commonly held dogma that an intact, fully functioning immune system is important for injury repair and better prognoses.

\section{Determine the efficacy of erlotinib as an injury-ameliorating strategy. Data} outlined in Chapter 4 indicate that inhibiting EGFR activation after one, low dose of cisplatin $(7 \mathrm{mg} / \mathrm{kg})$ exacerbates kidney injury, leading to a severe AKI phenotype. This is not surprising as EGFR and its downstream signaling pathways are highly involved in initial injury repair after an insult to the kidney. 
Thus, blocking the activation of EGFR means that injury cannot be resolved. In contrast, sustained activation of EGFR promotes the development of fibrosis. At Day 24, EGFR is still activated in cancer mice treated with cisplatin. Thus, using erlotinib to block fibrotic processes would provide a novel strategy for intervention during cisplatin-induced kidney injury. In order to test this, we are currently treating cancer mice with our repeated dosing regimen of cisplatin. Starting after the fourth dose of cisplatin, mice were administered $25 \mathrm{mg} / \mathrm{kg}$ erlotinib once a day for a month (Fig 5.2). We hypothesize that mice that received erlotinib post-cisplatin treatment will have less fibrosis. Thus, erlotinib may have utility as a preventative measure from the development of CKD.

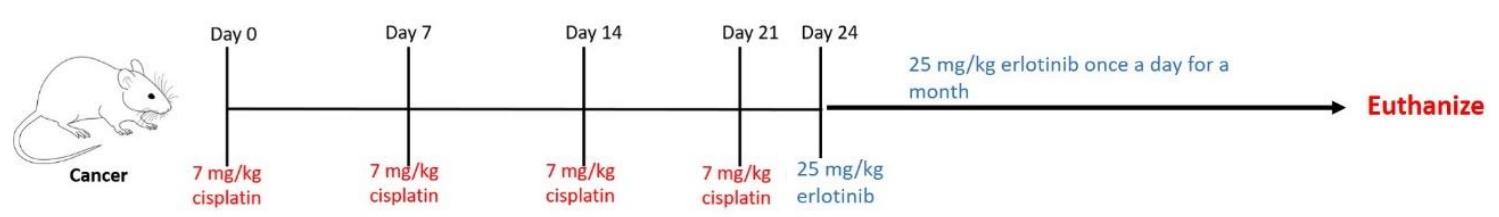

Figure 5.2. Erlotinib after repeated dosing of cisplatin. Mice with cancer were administered cisplatin $(7 \mathrm{mg} / \mathrm{kg})$ once a week for four weeks. At Day $24,25 \mathrm{mg} / \mathrm{kg}$ erlotinib was administered once a day for a month.

\section{Explore the role of circadian dysregulation in cisplatin-induced kidney} injury, especially in the context of cancer. Using the same transgenic mouse model of lung adenocarcinoma discussed in Chapter 4, Masri et al found that lung adenocarcinoma operated as an endogenous reorganizer of circadian functions, specifically liver metabolism (129). The function of the kidney is also tightly controlled by normal circadian rhythms. In addition, cisplatin has been a subject 
of several chronopharmacological studies. Lèvi et al reported that administration of cisplatin in the late afternoon after doxorubicin versus the morning after doxorubicin resulted in less nephrotoxicity with no effect on therapeutic efficacy (130). Furthermore, Li et al found that patients with NSCLC that received cisplatin in the late evening had less neutropenia, gastrointestinal toxicity, and leucopenia that coincided with higher rate of cisplatin clearance at this time point (131). Combining these studies, we hope to understand if circadian rhythms in the kidney are altered with cancer, and how this may have an effect on kidney injury, particularly the development of fibrosis, during the course of cisplatin treatment. Preliminary data indicate that repeated administration of cisplatin causes a switch in expression levels in the core clock protein- BMAL1 (Fig 5.3A). Furthermore, BMAL1 levels are altered basally in cancer mice (Fig 5.3B). Understanding how these changes may alter injury outcomes will provide valuable insight into using the time of day as a way to find a balance between therapeutic efficacy and decreasing nephrotoxic effects of cisplatin. 

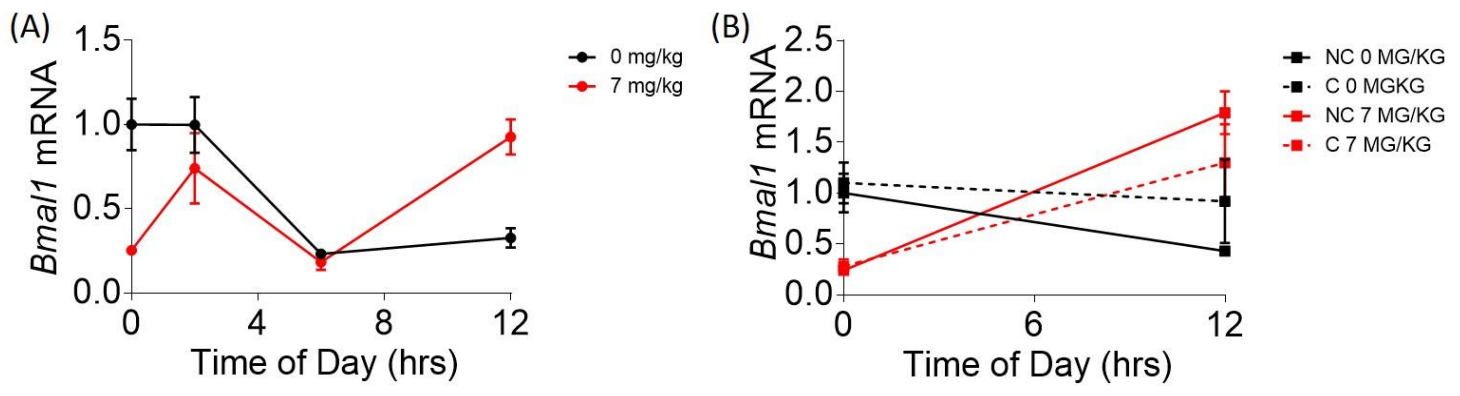

Figure 5.3. Alterations in BMAL1, an essential regulator of circadian rhythms.

(A) Levels of Bmall measured via QRTPCR in kidneys treated with saline or cisplatin at various times of day. (B) Bmall levels measured in non cancer and cancer mice treated at either 6 am (time of day $=0$ ) or $6 \mathrm{pm}$ (time of day=12). 


\section{REFERENCES}

1. Ferenbach DA, Bonventre JV. Mechanisms of maladaptive repair after AKI leading to accelerated kidney ageing and CKD. Nature reviews Nephrology. 2015;11(5):264-76. doi: 10.1038/nrneph.2015.3. PubMed PMID: 25643664; PMCID: 4412815.

2. Cohen SM, Lippard SJ. Cisplatin: from DNA damage to cancer chemotherapy. Progress in nucleic acid research and molecular biology. 2001;67:93-130. PubMed PMID: 11525387.

3. Eustace P. History and development of cisplatin in the management of malignant disease. Cancer Nurs. 1980;3(5):373-8. PubMed PMID: 6902674.

4. Rosenberg B, Van Camp L, Grimley EB, Thomson AJ. The inhibition of growth or cell division in Escherichia coli by different ionic species of platinum(IV) complexes. J Biol Chem. 1967;242(6):1347-52. PubMed PMID: 5337590.

5. Rosenberg B, Vancamp L, Krigas T. Inhibition of Cell Division in Escherichia Coli by Electrolysis Products from a Platinum Electrode. Nature. 1965;205:698-9. PubMed PMID: 14287410.

6. Wang D, Lippard SJ. Cellular processing of platinum anticancer drugs. Nature reviews Drug discovery. 2005;4(4):307-20. doi: 10.1038/nrd1691. PubMed PMID: 15789122.

7. Rose PG. Amifostine cytoprotection with chemotherapy for advanced ovarian carcinoma. Seminars in oncology. 1996;23(4 Suppl 8):83-9. PubMed PMID: 87836 
8. Siddik ZH. Cisplatin: mode of cytotoxic action and molecular basis of resistance. Oncogene. 2003;22(47):7265-79. doi: 10.1038/sj.onc.1206933. PubMed PMID: 14576837.

9. Pabla N, Dong Z. Cisplatin nephrotoxicity: Mechanisms and renoprotective strategies. Kidney Int. 2008;73(9):994-1007. doi: DOI 10.1038/sj.ki.5002786. PubMed PMID: WOS:000254960000005.

10. Ozkok A, Edelstein CL. Pathophysiology of cisplatin-induced acute kidney injury. BioMed research international. 2014;2014:967826. doi: 10.1155/2014/967826. PubMed PMID: 25165721; PMCID: 4140112.

11. Ciarimboli G, Ludwig T, Lang D, Pavenstadt H, Koepsell H, Piechota HJ, Haier J, Jaehde U, Zisowsky J, Schlatter E. Cisplatin nephrotoxicity is critically mediated via the human organic cation transporter 2. The American journal of pathology. 2005;167(6):1477-84. doi: 10.1016/S0002-9440(10)61234-5. PubMed PMID: 16314463; PMCID: 1613191.

12. Ludwig T, Riethmuller C, Gekle M, Schwerdt G, Oberleithner H. Nephrotoxicity of platinum complexes is related to basolateral organic cation transport. Kidney Int. 2004;66(1):196-202. doi: 10.1111/j.1523-1755.2004.00720.x. PubMed PMID: 15200426. 13. Yonezawa A, Masuda S, Nishihara K, Yano I, Katsura T, Inui K. Association between tubular toxicity of cisplatin and expression of organic cation transporter rOCT2 (Slc22a2) in the rat. Biochemical pharmacology. 2005;70(12):1823-31. doi: 10.1016/j.bcp.2005.09.020. PubMed PMID: 16242669. 
14. Arany I, Safirstein RL. Cisplatin nephrotoxicity. Semin Nephrol. 2003;23(5):4604. PubMed PMID: 13680535.

15. Miller RP, Tadagavadi RK, Ramesh G, Reeves WB. Mechanisms of Cisplatin nephrotoxicity. Toxins. 2010;2(11):2490-518. doi: 10.3390/toxins2112490. PubMed PMID: 22069563; PMCID: 3153174.

16. Lee RH, Song JM, Park MY, Kang SK, Kim YK, Jung JS. Cisplatin-induced apoptosis by translocation of endogenous Bax in mouse collecting duct cells.

Biochemical pharmacology. 2001;62(8):1013-23. PubMed PMID: 11597570.

17. Ramesh G, Reeves WB. TNF-alpha mediates chemokine and cytokine expression and renal injury in cisplatin nephrotoxicity. The Journal of clinical investigation. 2002;110(6):835-42. doi: 10.1172/JCI15606. PubMed PMID: 12235115; PMCID: 151130.

18. Zhang B, Ramesh G, Norbury CC, Reeves WB. Cisplatin-induced nephrotoxicity is mediated by tumor necrosis factor-alpha produced by renal parenchymal cells. Kidney Int. 2007;72(1):37-44. doi: 10.1038/sj.ki.5002242. PubMed PMID: 17396112.

19. Ricci Z, Cruz D, Ronco C. The RIFLE classification for acute kidney injury definition. American journal of surgery. 2009;198(1):152-3. doi:

10.1016/j.amjsurg.2008.06.033. PubMed PMID: 18805517.

20. Van Biesen W, Vanholder R, Lameire N. Defining acute renal failure: RIFLE and beyond. Clinical journal of the American Society of Nephrology : CJASN.

2006;1(6):1314-9. doi: 10.2215/CJN.02070606. PubMed PMID: 17699363.

21. Uchino S, Kellum JA, Bellomo R, Doig GS, Morimatsu H, Morgera S, Schetz M, Tan I, Bouman C, Macedo E, Gibney N, Tolwani A, Ronco C, Beginning, Ending 
Supportive Therapy for the Kidney I. Acute renal failure in critically ill patients: a multinational, multicenter study. Jama. 2005;294(7):813-8. doi: 10.1001/jama.294.7.813. PubMed PMID: 16106006.

22. De Corte W, Dhondt A, Vanholder R, De Waele J, Decruyenaere J, Sergoyne V, Vanhalst J, Claus S, Hoste EA. Long-term outcome in ICU patients with acute kidney injury treated with renal replacement therapy: a prospective cohort study. Crit Care. 2016;20(1):256. doi: 10.1186/s13054-016-1409-z. PubMed PMID: 27520553; PMCID: PMC4983760.

23. Hoste EA, De Corte W. AKI patients have worse long-term outcomes, especially in the immediate post-ICU period. Crit Care. 2012;16(4):148. doi: 10.1186/cc11470. PubMed PMID: 22958588; PMCID: PMC3580744.

24. Greenberg JH, Coca S, Parikh CR. Long-term risk of chronic kidney disease and mortality in children after acute kidney injury: a systematic review. BMC Nephrol. 2014;15:184. doi: 10.1186/1471-2369-15-184. PubMed PMID: 25416588; PMCID: PMC4251927.

25. Coca SG, Yusuf B, Shlipak MG, Garg AX, Parikh CR. Long-term risk of mortality and other adverse outcomes after acute kidney injury: a systematic review and meta-analysis. Am J Kidney Dis. 2009;53(6):961-73. doi: 10.1053/j.ajkd.2008.11.034. PubMed PMID: 19346042; PMCID: PMC2726041.

26. Chawla LS, Amdur RL, Amodeo S, Kimmel PL, Palant CE. The severity of acute kidney injury predicts progression to chronic kidney disease. Kidney Int. 2011;79(12):1361-9. doi: 10.1038/ki.2011.42. PubMed PMID: 21430640; PMCID: 3257034. 
27. Coresh J, Selvin E, Stevens LA, Manzi J, Kusek JW, Eggers P, Van Lente F, Levey AS. Prevalence of chronic kidney disease in the United States. Jama. 2007;298(17):2038-47. doi: 10.1001/jama.298.17.2038. PubMed PMID: 17986697. 28. Rifkin DE, Coca SG, Kalantar-Zadeh K. Does AKI truly lead to CKD? J Am Soc Nephrol. 2012;23(6):979-84. doi: 10.1681/ASN.2011121185. PubMed PMID: 22460531; PMCID: PMC3358766.

29. Varrier M, Forni LG, Ostermann M. Long-term sequelae from acute kidney injury: potential mechanisms for the observed poor renal outcomes. Crit Care. 2015;19:102. doi: 10.1186/s13054-015-0805-0. PubMed PMID: 25887052; PMCID: PMC4361133.

30. Sasaki T, Motoyama S, Komatsuda A, Shibata H, Sato Y, Yoshino K, Wakita A, Saito H, Anbai A, Jin M, Minamiya Y. Two cases of cisplatin-induced permanent renal failure following neoadjuvant chemotherapy for esophageal cancer. Int J Surg Case Rep. 2016;20:63-7. doi: 10.1016/j.ijscr.2016.01.009. PubMed PMID: 26851395; PMCID: PMC4818298.

31. Arga M, Oguz A, Pinarli FG, Karadeniz C, Citak EC, Emeksiz HC, Duran EA, Soylemezoglu O. Risk factors for cisplatin-induced long-term nephrotoxicity in pediatric cancer survivors. Pediatr Int. 2015;57(3):406-13. doi: 10.1111/ped.12542. PubMed PMID: 25441241.

32. Skinner R, Parry A, Price L, Cole M, Craft AW, Pearson AD. Persistent nephrotoxicity during 10-year follow-up after cisplatin or carboplatin treatment in childhood: relevance of age and dose as risk factors. European journal of cancer. 2009;45(18):3213-9. doi: 10.1016/j.ejca.2009.06.032. PubMed PMID: 19850470. 
33. Lee S, Huen S, Nishio H, Nishio S, Lee HK, Choi BS, Ruhrberg C, Cantley LG. Distinct macrophage phenotypes contribute to kidney injury and repair. J Am Soc Nephrol. 2011;22(2):317-26. doi: 10.1681/ASN.2009060615. PubMed PMID: 21289217; PMCID: PMC3029904.

34. Lech M, Grobmayr R, Ryu M, Lorenz G, Hartter I, Mulay SR, Susanti HE, Kobayashi KS, Flavell RA, Anders HJ. Macrophage phenotype controls long-term AKI outcomes--kidney regeneration versus atrophy. J Am Soc Nephrol. 2014;25(2):292-304. doi: 10.1681/ASN.2013020152. PubMed PMID: 24309188; PMCID: PMC3904561. 35. Kinsey GR. Macrophage dynamics in AKI to CKD progression. J Am Soc Nephrol. 2014;25(2):209-11. doi: 10.1681/ASN.2013101110. PubMed PMID: 24309186; PMCID: PMC3904577.

36. Grgic I, Campanholle G, Bijol V, Wang C, Sabbisetti VS, Ichimura T, Humphreys BD, Bonventre JV. Targeted proximal tubule injury triggers interstitial fibrosis and glomerulosclerosis. Kidney Int. 2012;82(2):172-83. doi: 10.1038/ki.2012.20. PubMed PMID: 22437410; PMCID: 3480325.

37. Yang L, Besschetnova TY, Brooks CR, Shah JV, Bonventre JV. Epithelial cell cycle arrest in G2/M mediates kidney fibrosis after injury. Nat Med. 2010;16(5):535-43, 1p following 143. doi: 10.1038/nm.2144. PubMed PMID: 20436483; PMCID: PMC3928013.

38. Levey AS, Coresh J. Chronic kidney disease. Lancet. 2012;379(9811):165-80. doi: 10.1016/S0140-6736(11)60178-5. PubMed PMID: 21840587.

39. Culy CR, Spencer CM. Amifostine: an update on its clinical status as a cytoprotectant in patients with cancer receiving chemotherapy or radiotherapy and its 
potential therapeutic application in myelodysplastic syndrome. Drugs. 2001;61(5):64184. PubMed PMID: 11368288.

40. Kemp G, Rose P, Lurain J, Berman M, Manetta A, Roullet B, Homesley H, Belpomme D, Glick J. Amifostine pretreatment for protection against cyclophosphamideinduced and cisplatin-induced toxicities: results of a randomized control trial in patients with advanced ovarian cancer. Journal of clinical oncology : official journal of the American Society of Clinical Oncology. 1996;14(7):2101-12. PubMed PMID: 8683243.

41. Sastry J, Kellie SJ. Severe neurotoxicity, ototoxicity and nephrotoxicity following high-dose cisplatin and amifostine. Pediatric hematology and oncology. 2005;22(5):4415. doi: 10.1080/08880010590964381. PubMed PMID: 16020136.

42. Weij1 NI, Elsendoorn TJ, Lentjes EG, Hopman GD, Wipkink-Bakker A, Zwinderman AH, Cleton FJ, Osanto S. Supplementation with antioxidant micronutrients and chemotherapy-induced toxicity in cancer patients treated with cisplatin-based chemotherapy: a randomised, double-blind, placebo-controlled study. European journal of cancer. 2004;40(11):1713-23. doi: 10.1016/j.ejca.2004.02.029. PubMed PMID: 15251161.

43. Gallagher KM, O'Neill S, Harrison EM, Ross JA, Wigmore SJ, Hughes J. Recent early clinical drug development for acute kidney injury. Expert Opin Investig Drugs. 2017;26(2):141-54. doi: 10.1080/13543784.2017.1274730. PubMed PMID: 27997816. 44. Okusa MD, Molitoris BA, Palevsky PM, Chinchilli VM, Liu KD, Cheung AK, Weisbord SD, Faubel S, Kellum JA, Wald R, Chertow GM, Levin A, Waikar SS, Murray PT, Parikh CR, Shaw AD, Go AS, Chawla LS, Kaufman JS, Devarajan P, Toto RM, Hsu CY, Greene TH, Mehta RL, Stokes JB, Thompson AM, Thompson BT, Westenfelder CS, 
Tumlin JA, Warnock DG, Shah SV, Xie Y, Duggan EG, Kimmel PL, Star RA. Design of clinical trials in acute kidney injury: a report from an NIDDK workshop--prevention trials. Clinical journal of the American Society of Nephrology : CJASN. 2012;7(5):851-5. doi: 10.2215/CJN.12811211. PubMed PMID: 22442188.

45. Zuk A, Palevsky PM, Fried L, Harrell FE, Jr., Khan S, McKay DB, Devey L, Chawla L, de Caestecker M, Kaufman JS, Thompson BT, Agarwal A, Greene T, Okusa MD, Bonventre JV, Dember LM, Liu KD, Humphreys BD, Gossett D, Xie Y, Norton JM, Kimmel PL, Star RA. Overcoming Translational Barriers in Acute Kidney Injury: A Report from an NIDDK Workshop. Clinical journal of the American Society of Nephrology : CJASN. 2018. doi: 10.2215/CJN.06820617. PubMed PMID: 29523680.

46. Bouchard J, Soroko SB, Chertow GM, Himmelfarb J, Ikizler TA, Paganini EP, Mehta RL, Program to Improve Care in Acute Renal Disease Study G. Fluid accumulation, survival and recovery of kidney function in critically ill patients with acute kidney injury. Kidney Int. 2009;76(4):422-7. doi: 10.1038/ki.2009.159. PubMed PMID: 19436332.

47. Coca SG, Yalavarthy R, Concato J, Parikh CR. Biomarkers for the diagnosis and risk stratification of acute kidney injury: a systematic review. Kidney Int. 2008;73(9):1008-16. doi: 10.1038/sj.ki.5002729. PubMed PMID: 18094679.

48. Zhou H, Hewitt SM, Yuen PS, Star RA. Acute Kidney Injury Biomarkers Needs, Present Status, and Future Promise. Nephrol Self Assess Program. 2006;5(2):6371. PubMed PMID: 19096722; PMCID: PMC2603572.

49. Skrypnyk NI, Siskind LJ, Faubel S, de Caestecker MP. Bridging translation for acute kidney injury with better preclinical modeling of human disease. Am J Physiol 
Renal Physiol. 2016;310(10):F972-84. doi: 10.1152/ajprenal.00552.2015. PubMed PMID: 26962107; PMCID: PMC4889323.

50. Sharp CN, Doll MA, Dupre TV, Shah PP, Subathra M, Siow D, Arteel GE, Megyesi J, Beverly LJ, Siskind LJ. Repeated Administration of Low-Dose Cisplatin in Mice Induces Fibrosis. Am J Physiol Renal Physiol. 2016:ajprenal 00512 2015. doi: 10.1152/ajprenal.00512.2015. PubMed PMID: 26739893.

51. Sharp CN, Siskind LJ. Developing Better Mouse Models to Study CisplatinInduced Kidney Injury. Am J Physiol Renal Physiol. 2017:ajprenal 00285 2017. doi: 10.1152/ajprenal.00285.2017. PubMed PMID: 28724610.

52. NCI. SEER Stat Fact Sheets: Lung and Bronchus Cancer [Web Page]. NCI; [cited 2016 April 7]. Available from: http://seer.cancer.gov/statfacts/html/lungb.html.

53. Weinstein JR, Anderson S. The aging kidney: physiological changes. Advances in chronic kidney disease. 2010;17(4):302-7. doi: 10.1053/j.ackd.2010.05.002. PubMed PMID: 20610357; PMCID: 2901622.

54. Torres R, Velazquez H, Chang JJ, Levene MJ, Moeckel G, Desir GV, Safirstein R. Three-Dimensional Morphology by Multiphoton Microscopy with Clearing in a Model of Cisplatin-Induced CKD. J Am Soc Nephrol. 2016;27(4):1102-12. doi: 10.1681/ASN.2015010079. PubMed PMID: 26303068; PMCID: PMC4814184. 55. Ravichandran K, Wang Q, Ozkok A, Jani A, Li H, He Z, Ljubanovic D, WeiserEvans MC, Nemenoff RA, Edelstein CL. CD4 T cell knockout does not protect against kidney injury and worsens cancer. J Mol Med (Berl). 2016;94(4):443-55. doi: 10.1007/s00109-015-1366-z. PubMed PMID: 26620676. 
56. Katagiri D, Hamasaki Y, Doi K, Negishi K, Sugaya T, Nangaku M, Noiri E. Interstitial renal fibrosis due to multiple cisplatin treatments is ameliorated by semicarbazide-sensitive amine oxidase inhibition. Kidney Int. 2016;89(2):374-85. doi: 10.1038/ki.2015.327. PubMed PMID: 26535996.

57. Coca SG, Singanamala S, Parikh CR. Chronic kidney disease after acute kidney injury: a systematic review and meta-analysis. Kidney Int. 2012;81(5):442-8. doi:

10.1038/ki.2011.379. PubMed PMID: 22113526; PMCID: PMC3788581.

58. Chawla LS, Eggers PW, Star RA, Kimmel PL. Acute Kidney Injury and Chronic Kidney Disease as Interconnected Syndromes. New Engl J Med. 2014;371(1):58-66. doi: Doi 10.1056/Nejmra1214243. PubMed PMID: WOS:000338265700010.

59. Sugimoto H, Grahovac G, Zeisberg M, Kalluri R. Renal fibrosis and glomerulosclerosis in a new mouse model of diabetic nephropathy and its regression by bone morphogenic protein-7 and advanced glycation end product inhibitors. Diabetes. 2007;56(7):1825-33. doi: 10.2337/db06-1226. PubMed PMID: 17456853.

60. Jo SK, Rosner MH, Okusa MD. Pharmacologic treatment of acute kidney injury: why drugs haven't worked and what is on the horizon. Clinical journal of the American Society of Nephrology : CJASN. 2007;2(2):356-65. doi: 10.2215/CJN.03280906. PubMed PMID: 17699435.

61. Fogo AB. Mechanisms of progression of chronic kidney disease. Pediatr Nephrol. 2007;22(12):2011-22. doi: 10.1007/s00467-007-0524-0. PubMed PMID: 17647026; PMCID: PMC2064942.

62. Noto A, Cibecchini F, Fanos V, Mussap M. NGAL and metabolomics: the single biomarker to reveal the metabolome alterations in kidney injury. BioMed research 
international. 2013;2013:612032. doi: 10.1155/2013/612032. PubMed PMID: 23607092; PMCID: PMC3625560.

63. Devarajan P. Neutrophil gelatinase-associated lipocalin: a promising biomarker for human acute kidney injury. Biomark Med. 2010;4(2):265-80. doi: 10.2217/bmm.10.12. PubMed PMID: 20406069; PMCID: PMC2893148.

64. Fielding CA, Jones GW, McLoughlin RM, McLeod L, Hammond VJ, Uceda J, Williams AS, Lambie M, Foster TL, Liao CT, Rice CM, Greenhill CJ, Colmont CS, Hams E, Coles B, Kift-Morgan A, Newton Z, Craig KJ, Williams JD, Williams GT, Davies SJ, Humphreys IR, O'Donnell VB, Taylor PR, Jenkins BJ, Topley N, Jones SA. Interleukin-6 signaling drives fibrosis in unresolved inflammation. Immunity. 2014;40(1):40-50. doi: 10.1016/j.immuni.2013.10.022. PubMed PMID: 24412616; PMCID: PMC3919204.

65. Meng XM, Nikolic-Paterson DJ, Lan HY. Inflammatory processes in renal fibrosis. Nature reviews Nephrology. 2014;10(9):493-503. doi:

10.1038/nrneph.2014.114. PubMed PMID: 24981817.

66. Gupta J, Mitra N, Kanetsky PA, Devaney J, Wing MR, Reilly M, Shah VO, Balakrishnan VS, Guzman NJ, Girndt M, Periera BG, Feldman HI, Kusek JW, Joffe MM, Raj DS, Investigators CS. Association between albuminuria, kidney function, and inflammatory biomarker profile in CKD in CRIC. Clinical journal of the American Society of Nephrology : CJASN. 2012;7(12):1938-46. doi: 10.2215/CJN.03500412. PubMed PMID: 23024164; PMCID: PMC3513744.

67. Ramesh G, Kimball SR, Jefferson LS, Reeves WB. Endotoxin and cisplatin synergistically stimulate TNF-alpha production by renal epithelial cells. Am J Physiol 
Renal Physiol. 2007;292(2):F812-9. doi: 10.1152/ajprenal.00277.2006. PubMed PMID: 17032936.

68. Pan B, Liu G, Jiang Z, Zheng D. Regulation of renal fibrosis by macrophage polarization. Cellular physiology and biochemistry : international journal of experimental cellular physiology, biochemistry, and pharmacology. 2015;35(3):1062-9. doi: 10.1159/000373932. PubMed PMID: 25662173.

69. Ricardo SD, van Goor H, Eddy AA. Macrophage diversity in renal injury and repair. The Journal of clinical investigation. 2008;118(11):3522-30. doi:

10.1172/JCI36150. PubMed PMID: 18982158; PMCID: PMC2575702.

70. Kim MG, Kim SC, Ko YS, Lee HY, Jo SK, Cho W. The Role of M2 Macrophages in the Progression of Chronic Kidney Disease following Acute Kidney Injury. PLoS One. 2015;10(12):e0143961. doi: 10.1371/journal.pone.0143961. PubMed PMID: 26630505; PMCID: PMC4667939.

71. Sindrilaru A, Peters T, Wieschalka S, Baican C, Baican A, Peter H, Hainzl A, Schatz S, Qi Y, Schlecht A, Weiss JM, Wlaschek M, Sunderkotter C, ScharffetterKochanek K. An unrestrained proinflammatory M1 macrophage population induced by iron impairs wound healing in humans and mice. The Journal of clinical investigation. 2011;121(3):985-97. doi: 10.1172/JCI44490. PubMed PMID: 21317534; PMCID: PMC3049372.

72. Lisi L, Ciotti GM, Braun D, Kalinin S, Curro D, Dello Russo C, Coli A, Mangiola A, Anile C, Feinstein DL, Navarra P. Expression of iNOS, CD163 and ARG-1 taken as M1 and M2 markers of microglial polarization in human glioblastoma and the 
surrounding normal parenchyma. Neurosci Lett. 2017;645:106-12. doi:

10.1016/j.neulet.2017.02.076. PubMed PMID: 28259657.

73. Thakar CV, Christianson A, Himmelfarb J, Leonard AC. Acute kidney injury episodes and chronic kidney disease risk in diabetes mellitus. Clinical journal of the American Society of Nephrology : CJASN. 2011;6(11):2567-72. doi:

10.2215/CJN.01120211. PubMed PMID: 21903988; PMCID: 3359576.

74. Tang J, Liu N, Tolbert E, Ponnusamy M, Ma L, Gong R, Bayliss G, Yan H, Zhuang S. Sustained activation of EGFR triggers renal fibrogenesis after acute kidney injury. The American journal of pathology. 2013;183(1):160-72. doi:

10.1016/j.ajpath.2013.04.005. PubMed PMID: 23684791; PMCID: PMC3702747.

75. Schiffrin EL. Role of endothelin-1 in hypertension and vascular disease. Am J Hypertens. 2001;14(6 Pt 2):83S-9S. PubMed PMID: 11411770.

76. Su H, Lei CT, Zhang C. Interleukin-6 Signaling Pathway and Its Role in Kidney Disease: An Update. Front Immunol. 2017;8:405. doi: 10.3389/fimmu.2017.00405. PubMed PMID: 28484449; PMCID: PMC5399081.

77. Basile DP. The endothelial cell in ischemic acute kidney injury: implications for acute and chronic function. Kidney Int. 2007;72(2):151-6. doi: 10.1038/sj.ki.5002312. PubMed PMID: 17495858.

78. Deanfield JE, Halcox JP, Rabelink TJ. Endothelial function and dysfunction: testing and clinical relevance. Circulation. 2007;115(10):1285-95. doi:

10.1161/CIRCULATIONAHA.106.652859. PubMed PMID: 17353456.

79. Liu L, Shi GP. CD31: beyond a marker for endothelial cells. Cardiovasc Res. 2012;94(1):3-5. doi: 10.1093/cvr/cvs108. PubMed PMID: 22379038. 
80. Frankish H. 15 million new cancer cases per year by 2020, says WHO. Lancet. 2003;361(9365):1278. doi: 10.1016/S0140-6736(03)13038-3. PubMed PMID: 12699963.

81. Bellomo R, Kellum JA, Ronco C. Defining and classifying acute renal failure: from advocacy to consensus and validation of the RIFLE criteria. Intensive care medicine. 2007;33(3):409-13. doi: 10.1007/s00134-006-0478-x. PubMed PMID: 17165018.

82. Cruz DN, Ricci Z, Ronco C. Clinical review: RIFLE and AKIN--time for reappraisal. Crit Care. 2009;13(3):211. doi: 10.1186/cc7759. PubMed PMID: 19638179; PMCID: PMC2717405.

83. Chertow GM, Burdick E, Honour M, Bonventre JV, Bates DW. Acute kidney injury, mortality, length of stay, and costs in hospitalized patients. J Am Soc Nephrol. 2005;16(11):3365-70. doi: 10.1681/ASN.2004090740. PubMed PMID: 16177006.

84. Linder A, Fjell C, Levin A, Walley KR, Russell JA, Boyd JH. Small acute increases in serum creatinine are associated with decreased long-term survival in the critically ill. Am J Respir Crit Care Med. 2014;189(9):1075-81. doi:

10.1164/rccm.201311-2097OC. PubMed PMID: 24601781.

85. Tan HL, Yap JQ, Qian Q. Acute Kidney Injury: Tubular Markers and Risk for Chronic Kidney Disease and End-Stage Kidney Failure. Blood Purif. 2016;41(1-3):14450. doi: 10.1159/000441269. PubMed PMID: 26764483.

86. Slocum JL, Heung M, Pennathur S. Marking renal injury: can we move beyond serum creatinine? Transl Res. 2012;159(4):277-89. doi: 10.1016/j.trsl.2012.01.014. PubMed PMID: 22424431; PMCID: PMC3308350. 
87. Pecoits-Filho R, Lindholm B, Axelsson J, Stenvinkel P. Update on interleukin-6 and its role in chronic renal failure. Nephrol Dial Transplant. 2003;18(6):1042-5. PubMed PMID: 12748331.

88. Zhang W, Wang W, Yu H, Zhang Y, Dai Y, Ning C, Tao L, Sun H, Kellems RE, Blackburn MR, Xia Y. Interleukin 6 underlies angiotensin II-induced hypertension and chronic renal damage. Hypertension. 2012;59(1):136-44. doi:

10.1161/HYPERTENSIONAHA.111.173328. PubMed PMID: 22068875; PMCID: PMC3842011.

89. Marini JC, Keller B, Didelija IC, Castillo L, Lee B. Enteral arginase II provides ornithine for citrulline synthesis. Am J Physiol Endocrinol Metab. 2011;300(1):E188-94. doi: 10.1152/ajpendo.00413.2010. PubMed PMID: 20978229; PMCID: PMC3023202.

90. Morris SM, Jr., Bhamidipati D, Kepka-Lenhart D. Human type II arginase: sequence analysis and tissue-specific expression. Gene. 1997;193(2):157-61. PubMed PMID: 9256072.

91. Robbins ME, Bywaters TB, Jaenke RS, Hopewell JW, Matheson LM, Tothill P, Whitehouse E. Long-term studies of cisplatin-induced reductions in porcine renal functional reserve. Cancer Chemother Pharmacol. 1992;29(4):309-15. PubMed PMID: 1537078.

92. Robbins ME. Single injection techniques in determining age-related changes in porcine renal function. Int J Appl Radiat Isot. 1984;35(2):85-91. PubMed PMID: 6368408.

93. Swindle MM, Moody DC, Phillips LD. Swine as models in biomedical research. 1st. ed. Ames: Iowa State University Press; 1992. xv, 312 p. p. 
94. Forster R, Ancian P, Fredholm M, Simianer H, Whitelaw B, Steering Group of the RP. The minipig as a platform for new technologies in toxicology. Journal of pharmacological and toxicological methods. 2010;62(3):227-35. doi:

10.1016/j.vascn.2010.05.007. PubMed PMID: 20685311.

95. Sturmlechner I, Durik M, Sieben CJ, Baker DJ, van Deursen JM. Cellular senescence in renal ageing and disease. Nature reviews Nephrology. 2017;13(2):77-89. doi: 10.1038/nrneph.2016.183. PubMed PMID: 28029153.

96. Wen J, Zeng M, Shu Y, Guo D, Sun Y, Guo Z, Wang Y, Liu Z, Zhou H, Zhang W. Aging increases the susceptibility of cisplatin-induced nephrotoxicity. Age (Dordr). 2015;37(6):112. doi: 10.1007/s1 1357-015-9844-3. PubMed PMID: 26534724; PMCID: PMC5005850.

97. Perazella MA. Onco-nephrology: renal toxicities of chemotherapeutic agents. Clinical journal of the American Society of Nephrology : CJASN. 2012;7(10):1713-21. doi: 10.2215/CJN.02780312. PubMed PMID: 22879440.

98. Thyss A, Saudes L, Otto J, Creisson A, Gaspard MH, Dassonville O, Schneider M. Renal tolerance of cisplatin in patients more than 80 years old. Journal of clinical oncology : official journal of the American Society of Clinical Oncology. 1994;12(10):2121-5. doi: 10.1200/JCO.1994.12.10.2121. PubMed PMID: 7931482. 99. Hrushesky WJ, Shimp W, Kennedy BJ. Lack of age-dependent cisplatin nephrotoxicity. Am J Med. 1984;76(4):579-84. PubMed PMID: 6538750.

100. Abella V, Scotece M, Conde J, Gomez R, Lois A, Pino J, Gomez-Reino JJ, Lago F, Mobasheri A, Gualillo O. The potential of lipocalin-2/NGAL as biomarker for 
inflammatory and metabolic diseases. Biomarkers. 2015;20(8):565-71. doi:

10.3109/1354750X.2015.1123354. PubMed PMID: 26671823; PMCID: PMC4819811.

101. Jang HR, Park JH, Kwon GY, Park JB, Lee JE, Kim DJ, Kim YG, Kim SJ, Oh

HY, Huh W. Aging has small effects on initial ischemic acute kidney injury development despite changing intrarenal immunologic micromilieu in mice. Am J Physiol Renal Physiol. 2016;310(4):F272-83. doi: 10.1152/ajprenal.00217.2015. PubMed PMID: 26661651.

102. Lam AQ, Humphreys BD. Onco-nephrology: AKI in the cancer patient. Clinical journal of the American Society of Nephrology : CJASN. 2012;7(10):1692-700. doi:

10.2215/CJN.03140312. PubMed PMID: 22879433; PMCID: PMC3463207.

103. Christiansen CF, Johansen MB, Langeberg WJ, Fryzek JP, Sorensen HT. Incidence of acute kidney injury in cancer patients: a Danish population-based cohort study. Eur J Intern Med. 2011;22(4):399-406. doi: 10.1016/j.ejim.2011.05.005. PubMed PMID: 21767759.

104. Vogel P, Pletcher JM, Liang Y. Spontaneous acute tumor lysis syndrome as a cause of early deaths in short-term carcinogenicity studies using p53 +/- mice. Vet Pathol. 2010;47(4):719-24. doi: 10.1177/0300985810363484. PubMed PMID: 20435782.

105. Pabla N, Dong G, Jiang M, Huang S, Kumar MV, Messing RO, Dong Z. Inhibition of PKCdelta reduces cisplatin-induced nephrotoxicity without blocking chemotherapeutic efficacy in mouse models of cancer. The Journal of clinical investigation. 2011;121(7):2709-22. doi: 10.1172/JCI45586. PubMed PMID: 21633170; PMCID: 3223835. 
106. Ajzensztejn D, Hegde VS, Lee SM. Tumor lysis syndrome after treatment with docetaxel for non-small-cell lung cancer. Journal of clinical oncology : official journal of the American Society of Clinical Oncology. 2006;24(15):2389-91. doi:

10.1200/JCO.2005.02.8753. PubMed PMID: 16710040.

107. Beriwal S, Singh S, Garcia-Young JA. Tumor lysis syndrome in extensive-stage small-cell lung cancer. Am J Clin Oncol. 2002;25(5):474-5. PubMed PMID: 12393987. 108. Chen J, Chen JK, Nagai K, Plieth D, Tan M, Lee TC, Threadgill DW, Neilson EG, Harris RC. EGFR signaling promotes TGFbeta-dependent renal fibrosis. J Am Soc Nephrol. 2012;23(2):215-24. doi: 10.1681/ASN.2011070645. PubMed PMID: 22095949; PMCID: PMC3269185.

109. Samarakoon R, Dobberfuhl AD, Cooley C, Overstreet JM, Patel S, Goldschmeding R, Meldrum KK, Higgins PJ. Induction of renal fibrotic genes by TGFbeta1 requires EGFR activation, p53 and reactive oxygen species. Cell Signal.

2013;25(11):2198-209. doi: 10.1016/j.cellsig.2013.07.007. PubMed PMID: 23872073. 110. Andre F, Berrada N, Desmedt C. Implication of tumor microenvironment in the resistance to chemotherapy in breast cancer patients. Curr Opin Oncol. 2010;22(6):54751. doi: 10.1097/CCO.0b013e32833fb384. PubMed PMID: 20842030.

111. Tsai MJ, Chang WA, Huang MS, Kuo PL. Tumor microenvironment: a new treatment target for cancer. ISRN Biochem. 2014;2014:351959. doi:

10.1155/2014/351959. PubMed PMID: 25937967; PMCID: PMC4392996.

112. Floege J, Uhlig S. Kidney calling lung and call back: how organs talk to each other. Nephrol Dial Transplant. 2010;25(1):32-4. doi: 10.1093/ndt/gfp464. PubMed PMID: 19755472. 
113. Hoke TS, Douglas IS, Klein CL, He Z, Fang W, Thurman JM, Tao Y, Dursun B, Voelkel NF, Edelstein CL, Faubel S. Acute renal failure after bilateral nephrectomy is associated with cytokine-mediated pulmonary injury. J Am Soc Nephrol. 2007;18(1):155-64. doi: 10.1681/ASN.2006050494. PubMed PMID: 17167117.

114. Andres-Hernando A, Altmann C, Bhargava R, Okamura K, Bacalja J, Hunter B, Ahuja N, Soranno D, Faubel S. Prolonged acute kidney injury exacerbates lung inflammation at 7 days post-acute kidney injury. Physiol Rep. 2014;2(7). doi: 10.14814/phy2.12084. PubMed PMID: 25052489; PMCID: PMC4187574.

115. Chen J, Chen JK, Harris RC. Deletion of the epidermal growth factor receptor in renal proximal tubule epithelial cells delays recovery from acute kidney injury. Kidney Int. 2012;82(1):45-52. doi: 10.1038/ki.2012.43. PubMed PMID: 22418982; PMCID: PMC3376190.

116. Tang J, Liu N, Zhuang S. Role of epidermal growth factor receptor in acute and chronic kidney injury. Kidney Int. 2013;83(5):804-10. doi: 10.1038/ki.2012.435. PubMed PMID: 23325080; PMCID: PMC4626012.

117. Tang PA, Tsao MS, Moore MJ. A review of erlotinib and its clinical use. Expert Opin Pharmacother. 2006;7(2):177-93. doi: 10.1517/14656566.7.2.177. PubMed PMID: 16433583.

118. Cappuzzo F, Ciuleanu T, Stelmakh L, Cicenas S, Szczesna A, Juhasz E, Esteban E, Molinier O, Brugger W, Melezinek I, Klingelschmitt G, Klughammer B, Giaccone G, investigators S. Erlotinib as maintenance treatment in advanced non-small-cell lung cancer: a multicentre, randomised, placebo-controlled phase 3 study. Lancet Oncol. 2010;11(6):521-9. doi: 10.1016/S1470-2045(10)70112-1. PubMed PMID: 20493771. 
119. Duran I, Hotte SJ, Hirte H, Chen EX, MacLean M, Turner S, Duan L, Pond GR, Lathia C, Walsh S, Wright JJ, Dancey J, Siu LL. Phase I targeted combination trial of sorafenib and erlotinib in patients with advanced solid tumors. Clin Cancer Res. 2007;13(16):4849-57. doi: 10.1158/1078-0432.CCR-07-0382. PubMed PMID: 17699864.

120. Gatzemeier U, Pluzanska A, Szczesna A, Kaukel E, Roubec J, De Rosa F, Milanowski J, Karnicka-Mlodkowski H, Pesek M, Serwatowski P, Ramlau R, Janaskova T, Vansteenkiste J, Strausz J, Manikhas GM, Von Pawel J. Phase III study of erlotinib in combination with cisplatin and gemcitabine in advanced non-small-cell lung cancer: the Tarceva Lung Cancer Investigation Trial. Journal of clinical oncology : official journal of the American Society of Clinical Oncology. 2007;25(12):1545-52. doi:

10.1200/JCO.2005.05.1474. PubMed PMID: 17442998.

121. Wada Y, Iyoda M, Matsumoto K, Shindo-Hirai Y, Kuno Y, Yamamoto Y, Suzuki T, Saito T, Iseri K, Shibata T. Epidermal growth factor receptor inhibition with erlotinib partially prevents cisplatin-induced nephrotoxicity in rats. PLoS One.

2014;9(11):e111728. doi: 10.1371/journal.pone.0111728. PubMed PMID: 25390346;

PMCID: PMC4229108.

122. Izzedine H, Bahleda R, Khayat D, Massard C, Magne N, Spano JP, Soria JC. Electrolyte disorders related to EGFR-targeting drugs. Crit Rev Oncol Hematol. 2010;73(3):213-9. doi: 10.1016/j.critrevonc.2009.03.012. PubMed PMID: 19403315. 123. Jhaveri KD, Sakhiya V, Wanchoo R, Ross D, Fishbane S. Renal effects of novel anticancer targeted therapies: a review of the Food and Drug Administration Adverse 
Event Reporting System. Kidney Int. 2016;90(3):706-7. doi: 10.1016/j.kint.2016.06.027. PubMed PMID: 27521117.

124. Dimke H, van der Wijst J, Alexander TR, Meijer IM, Mulder GM, van Goor H, Tejpar S, Hoenderop JG, Bindels RJ. Effects of the EGFR Inhibitor Erlotinib on Magnesium Handling. J Am Soc Nephrol. 2010;21(8):1309-16. doi:

10.1681/ASN.2009111153. PubMed PMID: 20595681; PMCID: PMC2938592.

125. van Hennik MB, van der Vijgh WJ, Klein I, Elferink F, Vermorken JB, Winograd B, Pinedo HM. Comparative pharmacokinetics of cisplatin and three analogues in mice and humans. Cancer Res. 1987;47(23):6297-301. PubMed PMID: 3315183.

126. Han X, Patters AB, Ito T, Azuma J, Schaffer SW, Chesney RW. Knockout of the TauT gene predisposes C57BL/6 mice to streptozotocin-induced diabetic nephropathy. PLoS One. 2015;10(1):e0117718. doi: 10.1371/journal.pone.0117718. PubMed PMID: 25629817; PMCID: PMC4309680.

127. Walkin L, Herrick SE, Summers A, Brenchley PE, Hoff CM, Korstanje R, Margetts PJ. The role of mouse strain differences in the susceptibility to fibrosis: a systematic review. Fibrogenesis Tissue Repair. 2013;6(1):18. doi: 10.1186/1755-1536-618. PubMed PMID: 24294831; PMCID: PMC3849643.

128. Kato N, Watanabe Y, Ohno Y, Inoue T, Kanno Y, Suzuki H, Okada H. Mapping quantitative trait loci for proteinuria-induced renal collagen deposition. Kidney Int. 2008;73(9):1017-23. doi: 10.1038/KI.2008.7. PubMed PMID: 18305463.

129. Masri S, Papagiannakopoulos T, Kinouchi K, Liu Y, Cervantes M, Baldi P, Jacks T, Sassone-Corsi P. Lung Adenocarcinoma Distally Rewires Hepatic Circadian 
Homeostasis. Cell. 2016;165(4):896-909. doi: 10.1016/j.cell.2016.04.039. PubMed PMID: 27153497; PMCID: PMC5373476.

130. Levi F, Benavides M, Chevelle C, Le Saunier F, Bailleul F, Misset JL, Regensberg C, Vannetzel JM, Reinberg A, Mathe G. Chemotherapy of advanced ovarian cancer with 4'-O-tetrahydropyranyl doxorubicin and cisplatin: a randomized phase II trial with an evaluation of circadian timing and dose-intensity. Journal of clinical oncology : official journal of the American Society of Clinical Oncology. 1990;8(4):705-14. doi: 10.1200/JCO.1990.8.4.705. PubMed PMID: 2179481.

131. Li J, Chen R, Ji M, Zou SL, Zhu LN. Cisplatin-based chronotherapy for advanced non-small cell lung cancer patients: a randomized controlled study and its pharmacokinetics analysis. Cancer Chemother Pharmacol. 2015;76(3):651-5. doi: 10.1007/s00280-015-2804-x. PubMed PMID: 26093951. 


\section{ABBREVIATIONS}

AKI
ANOVA
ARG-1
B2M
BCL2
Bmal1

BSA
BUN
CCSP-rTta
CD3
CD31

CDDP
CHOP

CKD
Col1a1
CTGF
Ctr1
Cxc11
DNA
ECM
(p)EGFR

ELISA
ER
ESRD
FDA
Fn1
GFR
HO-1
H\&E
IACUC

Acute kidney injury

Analysis of variance

Arginase-1

Beta-2-microglobulin

B-cell lymphoma 2

Aryl hydrocarbon receptor nuclear translocator-like protein 1

Bovine serum albumin

Blood urea nitrogen

Clara cell-specific promoter-reverse

Tetracycline transactivator

Cluster of differentiation-3

Platelet endothelial cell adhesion molecule-1

Cisplatin

CCAAT/enhancer-binding protein homologous protein

Chronic kidney disease Collagen type 1a1

Connective tissue growth factor

Copper uptake protein 1

$\mathrm{C}-\mathrm{X}-\mathrm{C}$ motif chemokine ligand 1

Deoxyribonucleic acid

Extracellular matrix

(phospho) Epidermal growth factor receptor

Enzyme-linked immunosorbent assay

Endoplasmic reticulum

End-stage renal disease

Food \& Drug Administration

Fibronectin 1

Glomerular filtration rate

Heme oxygenase-1

Hematoxylin \& Eosin

Institutional Animal Care \& Use

Committee 


\begin{tabular}{|c|c|}
\hline ICAM-1 & Intercellular adhesion molecule-1 \\
\hline ICU & Intensive care unit \\
\hline IHC & Immunohistochemistry \\
\hline IL6 & Interleukin 6 \\
\hline $\mathrm{I} / \mathrm{R}$ & Ischemia/reperfusion \\
\hline (p)JNK & (phospho) c-jun N-terminal kinase \\
\hline Kim-1 & Kidney injury molecule-1 \\
\hline $\mathrm{KO}$ & Knockout \\
\hline MATE 1 & Multidrug and toxin extrusion protein-1 \\
\hline Mcp-1 & Monocyte chemoattractant protein-1 \\
\hline mRNA & Messenger ribonucleic acid \\
\hline (p)NFкB & $\begin{array}{l}\text { (phospho) Nuclear factor kappa-light- } \\
\text { chain enhancer of activated B cells }\end{array}$ \\
\hline NGAL & Neutrophil gelatinase-associated lipocalin \\
\hline NSCLC & Non-small cell lung cancer \\
\hline OCT2 & Organic cation transporter 2 \\
\hline PAS & Periodic acid-Schiff \\
\hline PBS & Phosphate-buffered saline \\
\hline QRTPCR & $\begin{array}{l}\text { Quantitative reverse transcriptase } \\
\text { polymerase chain reaction }\end{array}$ \\
\hline RNA & Ribonucleic acid \\
\hline RIFLE & $\begin{array}{l}\text { Risk, injury, failure, loss of kidney } \\
\text { function, and end stage kidney disease }\end{array}$ \\
\hline RTKI & Receptor tyrosine kinase inhibitor \\
\hline $\mathrm{SCr}$ & Serum creatinine \\
\hline SEM & Standard error of mean \\
\hline Asma & Alpha smooth muscle actin \\
\hline $\mathrm{SR} / \mathrm{FG}$ & Sirius red/fast green \\
\hline TBS & Tris-buffered saline \\
\hline TGF $\beta(1)$ & Transforming growth factor beta(1) \\
\hline TIMP-1 & TIMP metallopeptidase inhibitor-1 \\
\hline TLS & Tumor lysis syndrome \\
\hline $\mathrm{TNF} \alpha$ & Tumor necrosis factor alpha \\
\hline VCAM-1 & Vascular adhesion molecule-1 \\
\hline VEGFR-2 & $\begin{array}{l}\text { Vascular endothelial growth factor } \\
\text { receptor- } 2\end{array}$ \\
\hline
\end{tabular}




\title{
CURRICULUM VITA
}

\author{
Cierra N. Sharp \\ 16 Highwood Place Louisville, KY 40206 \\ (606)331-1234 \\ cnshar01@louisville.edu \\ cnsharp13@gmail.com
}

\section{EDUCATION}

M.S. Pharmacology and Toxicology, 2016

University of Louisville

GPA: 3.92

B.A. Biology, 2013

Minor: Chemistry, Psychology

Transylvania University

Dean's List for 6 semesters, graduated cum laude with honors in Biology

GPA: 3.7 


\section{Current Education: Ph.D. Pharmacology and Toxicology}

University of Louisville

Expected graduation date: May 2018

\section{SPECIAL TRAINING AND WORKSHOPS ATTENDED}

\section{Vanderbilt Mouse Kidney Injury Workshop}

Vanderbilt University, August 2016

\section{PRESENTATIONS}

\section{Oral Presentations}

- Treating lung-adenocarcinoma-bearing mice with cisplatin causes worsened renal fibrosis that cannot be prevented with erlotinib American Physiological Society Kentucky Chapter Annual Meeting (April 2018)

- The effects of advanced age and cancer in a clinically-relevant mouse model of cisplatin nephrotoxicity.

Ohio Valley Society of Toxicology Annual Fall Meeting (October 2016)

- Developing a more clinically relevant mouse model to study cisplatin nephrotoxicity. James Graham Brown Cancer Center Cancer Colloquia (June 2016)

\section{Poster Presentations}


- Worsened renal fibrosis in Kras4bG12D lung adenocarcinoma-bearing mice treated with repeated dosing of cisplatin may be EGFR-mediated. Southeastern Regional Lipid Conference (November 2017)

- Worsened renal fibrosis in Kras4bG12D lung adenocarcinoma-bearing mice treated with repeated dosing of cisplatin may be EGFR-mediated. American Society of Nephrology Kidney Week (November 2017)

- Worsened renal fibrosis in Kras $4 b G 12 D$ lung adenocarcinoma-bearing mice treated with repeated dosing of cisplatin may be EGFR-mediated. Research!Louisville (September 2017)

- The effects of advanced age and cancer in a clinically-relevant mouse model of cisplatin nephrotoxicity. Southeastern Regional Lipid Conference (October 2016)

- The effects of advanced age and cancer in a clinically-relevant mouse model of cisplatin nephrotoxicity. Research!Louisville (October 2016)

- Developing a clinically relevant model of cisplatin induced nephrotoxicity. Southeastern Regional Lipid Conference (November 2015)

- Developing a clinically relevant model of cisplatin induced nephrotoxicity. American Society of Nephrology Kidney Week (November 2015)

- Developing a clinically relevant model of cisplatin induced nephrotoxicity. Research!Louisville (October 2015)

\section{PUBLICATIONS AND ABSTRACTS}

\section{Publications}


- Sharp CN, Doll MA, Megyesi J, Oropilla GB, Beverly LJ, and Siskind LJ. Subclinical Kidney Injury Induced by Repeated Cisplatin Administration Results in Progressive Chronic Kidney Disease. In Press.

- Sharp CN, Siskind LJ. Developing Better Mouse Models to Study CisplatinInduced Kidney Injury. American journal of physiology Renal physiology. 2017 Oct 1;313(4):F835-F841. doi: 10.1152/ajprenal.00285.2017.

- Sharp CN, Doll MA, Dupre TV, Shah PP, Subathra M, Siow D, Arteel GE, Megyesi J, Beverly LJ, Siskind LJ. Repeated Administration of Low-Dose Cisplatin in Mice Induces Fibrosis. American journal of physiology Renal physiology. 2016:ajprenal 00512 2015. doi: 10.1152/ajprenal.00512.2015. PubMed PMID: 26739893.

- Dupre TV, Doll MA, Shah PP, Sharp CN, Kiefer A, Scherzer MT, Saurabh K, Saforo D, Casson L, Arteel GE, Jenson AB, Megyesi J, Schnellman RG, Beverly LJ, Siskind LJ. Suramin protects from cisplatin-induced acute kidney injury. American journal of physiology Renal physiology. 2016:ajprenal 004332015. Doi: 10.1152/ajprenal.00433.2015. PubMed PMID: 26661653.

- Dupre TV, Sharp CN, Siskind LJ. Renal Toxicology/Nephrotoxicity of Cisplatin and other Chemotherapeutic Agents. Comprehensive Toxicology 3e.

- $\quad \underline{\text { Dupre TV}}, \underline{\text { Doll MA }}, \underline{\text { Shah PP, }}$ Sharp CN,$\underline{\text { Siow D, Megyesi J, Shayman }}$

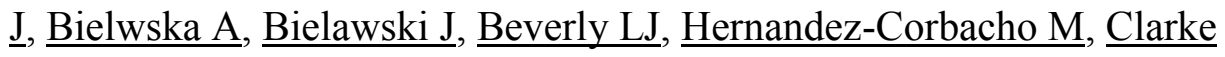
$\underline{\text { CJ}}, \underline{\text { Snider AJ, Schnellmann RG, }}$ Obeid LM, $\underline{\text { Hannun YA, }}$ Siskind LJ. Inhibiting glucosylceramide synthase exacerbates cisplatin-induced acute kidney injury. 
Journal of Lipid Research. DOI: 10.1194/j1r.M076745 PubMed PMID: 28490444.

\section{Abstracts}

- Jacobs, ON, Sharp CN, Doll MA, Megyesi J, Saforo DJ, Dupre TV, Beverly LJ, and Siskind LJ. Chronopharmacology studies in a clinically-relevant mouse model of cisplatin nephrotoxicity.

- Walls, KM, Sharp CN, Doll MA, Dupre TV, Beverly LJ, and Siskind LJ. Repeated administration of cisplatin increased EGFR/EGFR activation and renal fibrosis in Kras4bG12D lung adenocarcinoma-bearing mice, but kidney injury is further exacerbated with erlotinib/cisplatin combination treatment.

- Sharp CN, Doll MA, Dupre TV, Beverly LJ, and Siskind LJ. Worsened renal fibrosis in Kras4bG12D lung adenocarcinoma-bearing mice treated with repeated dosing of cisplatin may be EGFR-mediated.

- Sharp CN, Doll MA, Dupre TV, Beverly LJ, and Siskind LJ. Effects of comorbidities of age and cancer in a clinically-relevant mouse model of cisplatininduced kidney injury.

- Oropilla GB, Sharp CN, Doll MA, Siskind LJ. Strain differences in susceptibility to cisplatin-induced renal fibrosis.

- Lang AL, Kaelin BR, Yeo H, Sharp CN, Arteel GE, and Beier JI. Critical role of mammalian target of rapamycin (mTOR) in liver damage caused by VC metabolites in mice. 
- Dupre TV, Doll MA, Sharp CN, Siow D, Siskind LJ, Shah PP, Beverly LJ, Megyesi J, Shayman J, Bielawska A, Bielawski J. Buffering of ceramide through glucosylceramide synthase is protective of cisplatin-induced acute kidney injury.

- Sharp CN, Doll MA, Dupre TV, Siow D, Marimuthu S, Shah P, Beverly LJ, and Siskind LJ. Developing a clinically relevant model of cisplatin induced nephrotoxicity.

- Dupre TV, Doll MA, Shah PP, Sharp CN, Scherzer MT, Casson L, Megyesi J, Beverly LJ, Schnellmann RG, Siskind LJ. Suramin Protects from cisplatininduced acute kidney injury.

- Lang AL, Kaelin BR, Yeo H, Hudson SV, Poole LG, McKenzie CM, Sharp CN, Arteel GE, Beier JI. Critical role of mammalian target of rapamycin (mTOR) in liver damage caused by $\mathrm{VC}$ metabolites in mice.

\section{AWARDS AND SPECIAL RECOGNITION}

\section{8}

American Physiological Society Kentucky Chapter: First place- Graduate student platform presentation

- Research!Louisville: $2^{\text {nd }}$ place poster presentation for doctorate students

- University of Louisville: Graduate Student Council travel award to attend the 2017 American Society of Nephrology Kidney Week

2016 
- Ohio Valley Society of Toxicology (OVSOT): Givaudan Best PhD Student Platform Presentation Award

- American Society of Nephrology: Kidney Star 2016

- University of Louisville: Graduate Student Council travel award to attend the 2016 Vanderbilt Mouse Kidney Injury Workshop

2015

- Research!Louisville: $1^{\text {st }}$ place poster presentation for Master's students

- University of Louisville: Graduate Student Council travel award to present my work at American Society of Nephrology Kidney Week

2014

- University of Louisville: Graduate Student Council travel award to present my work at Southeastern regional lipid conference

- University of Louisville: IPIBS Fellowship covering tuition, benefits, and stipend

\section{PROFESSIONAL MEMBERSHIPS}

- American Society of Nephrology, 2015-present

- American Physiological Society, 2015-present

- Society of Toxicology, 2016-present

○ Graduate Student Leadership Committee, 2016-2017

- Ohio Valley Society of Toxicology, 2016-present

○ Graduate student representative, 2016-2017

\section{VOLUNTEER AND EXTRACURRICULAR ACTIVITIES}


2018

- Louisville Regional Science Fair Judge

2017

- Bio-Rad Science Ambassador

2015

- ExBEERiment volunteer

- Louisville Regional Science Fair Judge

2011

- Alpha Lambda Delta Service Chair (Transylvania University) 